\title{
WestVirginiaUniversity
}

THE RESEARCH REPOSITORY @ WVU

Graduate Theses, Dissertations, and Problem Reports

2004

\section{Operant conditioning in older adults with Alzheimer's disease}

\author{
Adam P. Spira \\ West Virginia University
}

Follow this and additional works at: https://researchrepository.wvu.edu/etd

\section{Recommended Citation}

Spira, Adam P., "Operant conditioning in older adults with Alzheimer's disease" (2004). Graduate Theses, Dissertations, and Problem Reports. 2125.

https://researchrepository.wvu.edu/etd/2125

This Dissertation is protected by copyright and/or related rights. It has been brought to you by the The Research Repository @ WVU with permission from the rights-holder(s). You are free to use this Dissertation in any way that is permitted by the copyright and related rights legislation that applies to your use. For other uses you must obtain permission from the rights-holder(s) directly, unless additional rights are indicated by a Creative Commons license in the record and/ or on the work itself. This Dissertation has been accepted for inclusion in WVU Graduate Theses, Dissertations, and Problem Reports collection by an authorized administrator of The Research Repository @ WVU.

For more information, please contact researchrepository@mail.wvu.edu. 


\title{
OPERANT CONDITIONING IN OLDER ADULTS WITH ALZHEIMER'S DISEASE
}

\author{
Adam P. Spira
}

Dissertation submitted to the Eberly College of Arts and Sciences of West Virginia University in partial fulfillment of the requirements for the degree of

\author{
Doctor of Philosophy \\ in \\ Psychology
}
Barry A. Edelstein, Ph.D. and Michael Perone, Ph.D., Chairs Marc W. Haut, Ph.D.
Deborah J. Jones, Ph.D.
Kevin T. Larkin, Ph.D.
Department of Psychology Morgantown, West Virginia 2004

Keywords: Operant Conditioning, Older Adults, Alzheimer's Disease

Copyright 2004, Adam P. Spira 


\title{
Abstract \\ Operant Conditioning in Older Adults with Alzheimer's Disease
}

\begin{abstract}
Adam P. Spira
Behavioral interventions are commonly implemented to manage agitation in older adults with dementia. However, the extent to which operant conditioning can occur in this population is unclear. The present study used a button-pressing task to evaluate the sensitivity of the responding of individuals with probable Alzheimer's disease to changes in schedules of reinforcement. Results indicate that operant conditioning can occur in older adults with AD. Specifically, three participants with AD exhibited responding that was highly sensitive to a transition from a fixed interval schedule to an extinction schedule. One participant's responding was sensitive to a more subtle transition. Comparison data is presented from older controls and college students. Clinical implications of findings and suggestions for future research are presented.
\end{abstract}




\section{Acknowledgements}

I owe a great debt of gratitude to Dr. Barry Edelstein for nurturing my initial interest in clinical geropsychology, and being a remarkable mentor and friend. Barry played a major role in making my graduate school experience stimulating and challenging. I very much appreciate his support and guidance during my graduate career at WVU, and his input during the conception and execution of this project. In addition, I would like to thank Dr. Michael Perone for acting as a dissertation co-chair with Barry, and for good-naturedly tolerating my many methodological questions and concerns. Thanks also to Dr. Marc Haut, Dr. Deborah Jones, and Dr. Kevin Larkin for their guidance as dissertation committee members. In addition, I would like to thank Dr. Christina Wilson for her assistance with participant recruitment.

I would like to thank Ryan Schiffbauer for his assistance with programming, and Anthony Rossetti for his contributions in programming and electrical engineering. These individuals' talents were critical to the project's completion. Thanks also to Sarah Foster, for assistance with data collection.

Many thanks to Melissa DeMore for her support, encouragement, and patience. I love you, Miss. Finally, thanks to Mom, Dad, Karen, and Daniel. I'm very fortunate to have such a loving and supportive family. 


\section{Table of Contents}

Title Page

Abstract

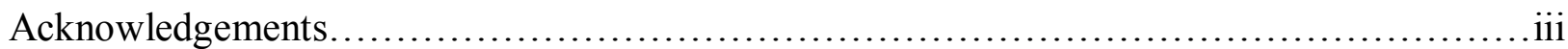

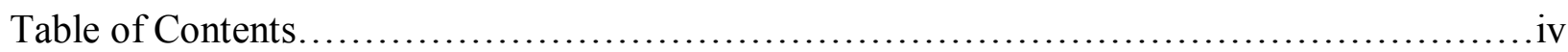

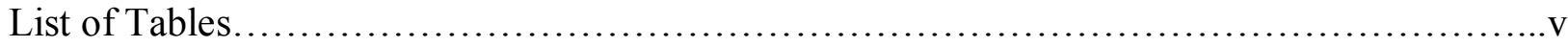

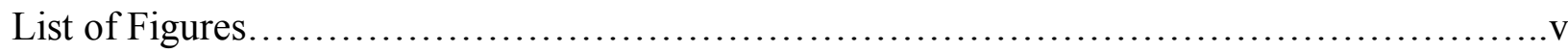

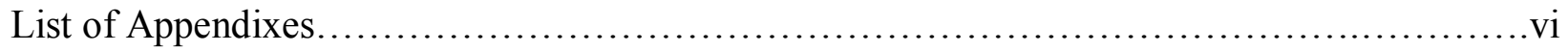

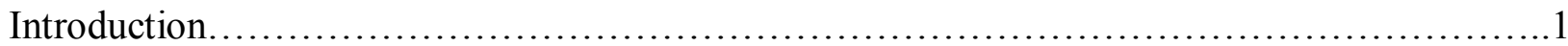

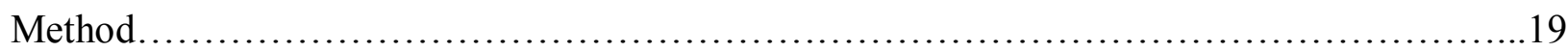

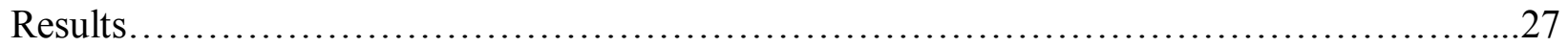

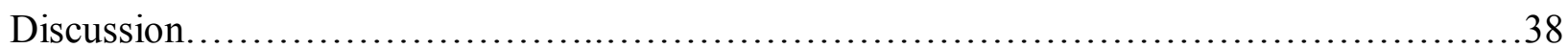

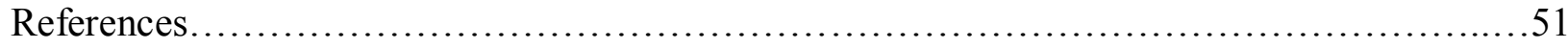

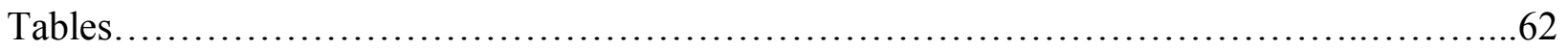

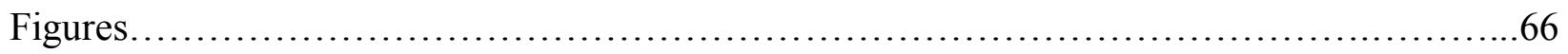

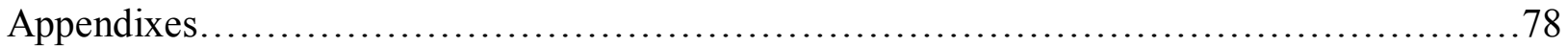


List of Tables

Table 1. Demographic and Psychosocial Information

Table 2. Participants' Raw and (z) Scores on CERAD Neuropsychological Tests

Table 3. Parameters for FR and FI Schedules, and Mean Response Rates Obtained by Participants Across Conditions

Table 4. Sensitivity Indices, Percent Change, and Retention Savings

\section{List of Figures}

Figure 1. Response rates of participant Y10 during initial and retention sessions.

Figure 2. Response rates of participant Y22 during initial session.

Figure 3. Response rates of participant Y21 during initial and retention sessions.

Figure 4. Response rates of participant Y17 during initial and retention sessions.

Figure 5. Response rates of participant Y18 during initial and retention sessions.

Figure 6. Response rates of participant OA27 during initial and retention sessions.

Figure 7. Response rates of participant OA13 during initial and retention sessions.

Figure 8. Response rates of participant OA20 during initial and retention sessions.

Figure 9. Response rates of participant OA26 during initial and retention sessions.

Figure 10. Response rates of participant AD23 during initial and retention sessions.

Figure 11. Response rates of participant AD30 during initial and retention sessions.

Figure 12. Response rates of participant AD31 during initial and retention sessions. 


\section{List of Appendixes}

Appendix A: NINCDS-ADRDA Diagnostic Criteria for Probable Alzheimer's Disease Appendix B: Clinical Interview

Appendix C: Cumulative record of participant Y10's responding during initial session. Appendix D: Cumulative record of participant Y22's responding during initial session. Appendix E: Cumulative record of participant Y21's responding during initial session. Appendix F: Cumulative record of participant Y17's responding during initial session. Appendix G: Cumulative record of participant Y18's responding during initial session. Appendix H: Cumulative record of participant OA27's responding during initial session. Appendix I: Cumulative record of participant OA13's responding during initial session. Appendix J: Cumulative record of participant OA20's responding during initial session. Appendix K: Cumulative record of participant OA26's responding during initial session. Appendix L: Cumulative record of participant AD23's responding during initial session. Appendix M: Cumulative record of participant AD30's responding during initial session. Appendix N: Cumulative record of participant AD31's responding during initial session. Appendix O: Cumulative record of participant AD28 during preliminary training and FR conditions. 
Operant Conditioning in Older Adults with Alzheimer's Disease

Alzheimer's disease (AD) is a degenerative brain disease that affects approximately four million Americans (Alzheimer's Association, 2002). AD primarily occurs in older adults; approximately $10 \%$ of adults over the age of 65 , and almost half of those above the age of 85 have the disease (Alzheimer's Association). AD is characterized by the accumulation of amyloid plaques and neurofibrillary tangles, structural abnormalities that are believed to impair normal brain function (Selkoe, 2002) and lead to the dementia associated with the disease (Kaplan \& Sadock, 1998). These abnormalities, as well as neuron loss, are observed in limbic structures such as hippocampus, amygdala, and nucleus basalis, and in association cortices responsible for memory and other high-level cognitive processes (Selkoe; Thompson, 2000).

$\mathrm{AD}$ is the most common cause of dementia (Alzheimer's Association), which the American Psychiatric Association (APA) defines as “. . . the development of multiple cognitive deficits that include memory impairment and ..." another cognitive disturbance from a list including aphasia (language disturbance), apraxia (motor disturbance), agnosia (inability to recognize objects), and impaired executive functioning (1994, p. 134). A diagnosis of dementia must reflect significant impairment in functioning and a decline from previous functional status (APA). $\mathrm{AD}$ is associated with a degenerative dementia leading to complete deterioration of functional capacity, and the eventual death of the affected individual. According to the National Center for Health Statistics, AD was among the ten most common causes of death in 1999 (Kochanek, Smith, \& Anderson, 2001).

\section{Agitation}

The organic pathology underlying AD can produce a variety of maladaptive behaviors, including aggression, screaming, and wandering. Researchers have attempted to categorize this 
disruptive behavior in various ways. These behaviors have typically been referred to as agitation. Cohen-Mansfield and Billig (1986) defined agitation as “. . . inappropriate verbal, vocal, or motor activity that is not explained by needs or confusion per se" (p. 712). Agitation includes behavior that is abusive or aggressive, occurs at an inappropriate frequency, or is socially inappropriate (Cohen-Mansfield and Billig). Although estimates of the prevalence of agitation vary widely across studies and settings, agitation is fairly common among older adults with dementia. Ballard and colleagues (2001) reported that 55\% of inpatients with dementia exhibited agitation. Cohen-Mansfield, Werner, Watson, and Pasis (1995) reported that approximately 63\% of the elderly at adult daycare centers exhibited agitation, and that these estimates rose to $90 \%$ when relatives were questioned about agitated behavior. Other authors estimated that 70 to $95 \%$ of older adults with dementia exhibited such behavior, depending upon setting (Chandler \& Chandler, 1988; Sourial, McCusker, Cole, \& Abrahamowicz, 2001; Swearer, Drachman, O’Donnell, \& Mitchell, 1988). Regardless of the exact prevalence figures, or the labels used to refer to it, it is clear that disruptive behavior is problematic for the disruptive individual, caregivers, and others in his or her immediate environment. Such behavior puts others at physical risk, and increases caregiver stress (Bourgeois, Schulz, \& Burgio, 1996). Thus, interventions to reduce agitation have the potential to improve quality of life for individuals with dementia, caregivers, and others in the disruptive individual's immediate environment.

\section{Behavioral Interventions for Disruptive Behavior}

Various approaches have been used to treat disruptive behavior in individuals with dementia, including psychodynamic interventions, reminiscence therapy, support groups, reality orientation, and behavioral approaches (Kasl-Godley \& Gatz, 2000). A number of interventions have utilized behavioral approaches, in which the antecedents and consequences of a target 
behavior are identified through functional assessment procedures, and then manipulated to change behavior. Such assessment procedures range from the relatively informal (e.g., direct observation of target behavior in naturalistic settings, interviews with informants) to experimental functional assessment procedures (e.g., functional analysis; Iwata et al., 1994). The clinician uses data collected through these assessment procedures to develop interventions that either modify antecedent conditions (to increase or decrease the probability of a given response), withhold reinforcing consequences (to decrease, or eliminate a behavior), or present reinforcing consequences in the absence of a target behavior or in the presence of an incompatible behavior (to increase the probability of an adaptive response). In addition, mildly aversive procedures might be used to punish target behaviors. Often, interventions strategically use the variables that are identified (through functional assessment) as maintaining or reinforcing a target behavior, in an attempt to increase or decrease that behavior (i.e., match treatment to function). For example, to reduce aggression that assessment reveals is maintained by attention, a clinician might withhold attention following aggression, and provide attention following a more adaptive response (e.g., asking for attention). Accurate functional assessment can play an important role in behavioral interventions. Failure to correctly identify the function of a behavior, or to match treatment to function as described, can result in an ineffective or even counterproductive intervention (Nelson \& Hayes, 1986).

A number of accounts of behavioral interventions for agitation in older adults with dementia have been published (e.g., Birchmore \& Clague, 1983; Bird, Alexopoulos, \& Adamowicz, 1995; Block, Boczkowski, Hansen, \& Vanderbeck, 1987; Boehm, Whall, Cosgrove, Locke, \& Schlenk, 1995; Haley, 1983; Hussian, 1982; Hussian \& Brown, 1987; Mayer \& Darby, 1991; Namazi, Rosner, \& Calkins, 1989). The results of these studies and reviews of the 
literature (Allen-Burge, Stevens, \& Burgio, 1999; Burgio \& Fisher, 2000; Fisher \& Carstensen, 1990; Kasl-Godley \& Gatz, 2000) suggest that clinicians be optimistic regarding the ability of behavioral interventions to reduce agitation in some older adults with dementia. However, the heterogeneity of both etiology and severity of dementia observed among participants in the applied literature hinders researchers' ability to draw conclusions regarding the ability of individuals with particular varieties of dementia to respond to behavioral interventions for disruptive behavior.

Basic research, conducted within a highly controlled environment with an etiologically homogeneous sample of older adults with dementia is needed to evaluate the degree to which the behavior of this population is amenable to behavior analytic interventions. A traditional, mechanized operant paradigm, in which the consequences of behavior are systematically manipulated, is the most appropriate methodological context for this research because it provides an excellent experimental analogue of the natural environment while allowing for maximal control of the variables that can threaten the validity of such studies, including experimenter error and bias in reinforcer delivery and recording of responding, and the countless other variables with which the natural environment is imbued (e.g., uncontrollable confounding reinforcers, noise, interruptions).

Although most basic laboratory investigations of operant behavior were originally conducted with non-human animals, a growing literature has been established involving the experimental analysis of human behavior, through methodologies analogous to those used with animals (cf. Baron \& Perone, 1982). The following section describes a number of methodological and conceptual issues associated with basic human operant research that are relevant to the present study, and includes a review of operant studies that have been conducted 
with both healthy older adults and older adults with dementia. This review is provided to establish a historical context for the present research.

\section{Human Operant Research}

Methodological and conceptual issues. A number of factors complicate operant research with humans. Specifically, Baron and Perone (1982) cautioned that lack of control over subjects' learning histories or access to reinforcers outside of the experimental session, as well as individual differences (e.g., age, gender, educational level) observed between human subjects threaten the validity of human operant studies. However, the authors pointed out that these obstacles do not preclude scientifically rigorous research; researchers can take steps to increase the similarity of their subjects and their histories. For example, between-subject individual differences can be reduced by studying individuals of the same gender, level of education, and age.

Another issue in human operant research is the degree of experimental control that can be demonstrated in light of the foregoing methodological limitations. More specifically, the question is how sensitive human subjects are to contingencies of reinforcement. Human operant studies have measured sensitivity by: (a) changing contingencies (across or within experimental sessions), and observing whether responding changes reliably following these manipulations (e.g., Hayes, Brownstein, Haas, \& Greenway, 1986; Hayes, Brownstein, Zettle, Rosenfarb, \& Korn, 1986; Shimoff, Matthews, \& Catania, 1986); or (b) evaluating the degree to which performance on concurrent schedules are consistent with that predicted by the matching law (Herrnstein, 1961, 1970; e.g., Dube \& McIlvane, 2002; Fisher \& Noll, 1996; Neef, Shea, \& Shade, 1992). In concurrent schedules, two or more response options (e.g., keys, buttons, levers) are simultaneously available to the subject, and each response option operates on a schedule of 
reinforcement that is independent of the other response options. According to the matching law, the distribution of a subject's responses among concurrent schedules of reinforcement is determined by the relative rates of reinforcement provided by each of these schedules (Hernnstein, 1961, 1970). In other words, the matching law predicts that a subject's response rates on one key or lever, relative to another, will be proportional to the relative rates of reinforcement provided by each response option.

Definitional problems have arisen concerning the construct of sensitivity, particularly when the responding of human and nonhuman subjects has been compared. One of the most striking findings of human operant research is that the responses of human subjects to particular schedules are frequently dissimilar to those exhibited by infrahuman animals (Buskist, Miller, \& Bennett, 1980; Kollins, Newland, \& Critchfield, 1997). Some researchers have referred to human operant behavior that differs from "schedule-typical nonhuman response patterns" as "insensitive" (Madden, Chase, \& Joyce, 1998, p. 2). Human verbal abilities have been proposed to account for these discrepancies between human and non-human responding (Hayes et al., 1986a; Madden et al.). Madden et al. concluded that, given these uniquely human verbal abilities and the methodological differences inherent to studying operant behavior across species, it is inappropriate to make interspecies comparisons regarding sensitivity (i.e., between human and nonhuman subjects' responding to particular schedules of reinforcement). Instead, the authors proposed a within-subject definition of sensitivity: "Sensitivity is demonstrated when an experimental manipulation affects behavior in an orderly and replicable manner. Insensitivity describes a lack of behavior change following an experimental manipulation" (p. 7). Madden and colleagues also emphasized that behavior that meets the above definition of sensitivity, but does not match an a priori, logically determined pattern of responding, should not be thought of as 
"insensitive." For example, even if response rate reliably increased in frequency following a change from a variable interval (VI) schedule to a differential reinforcement of low rates of responding (DRL) schedule, this responding would be considered sensitive to contingencies (regardless of the fact that it changed in rate in a direction contrary to what might have been logically expected). Age-Related Changes and Operant Research

Labouvie-Vief, Hoyer, Baltes, and Baltes (1974) proposed that operant procedures be used to measure the extent to which age-associated changes in human intellectual behavior are due to an irreversible process of decline. The authors emphasized the potential of the environment to influence intellectual behavior, and after explaining that the operant perspective is concerned with behaviors that are controlled by environmental events, wrote: "Consequently, to the extent that 'old' behavior assumes an operant status, it does not necessarily reflect intrinsic maturational processes" (p. 264). In other words, behavior that can be brought under environmental control can be thought of as being spared by age-related cognitive decline.

Operant research comparing healthy older and younger adults. Several researchers have examined age-related differences in operant conditioning. For example, Perone and Baron (1982) compared the effect of pacing (i.e., experimental imposition of time constraints) on the performance of 3 healthy younger adults and 3 healthy older adults on a task in which keys had to be pressed in a specific order. Specifically, pushing a "start" switch allowed participants to begin pressing keys to complete a 10-response sequence, which remained consistent during what was called the "performance" component and varied during the "acquisition" component (in which a new sequence was introduced each time). Errors produced a 5-s time-out, during which the apparatus was deactivated. Following successful completion of the sequence, a green light 
signaled that monetary reinforcement was available. At this point, the pressing of the "report" button activated a tone, which indicated that a sum of money was earned. Participants were required to press the "start" button to begin another sequence. Results indicated that during unpaced trials (in which there were no time restrictions), older men took longer to learn the sequence. Responding during paced (i.e., timed) components that required the acquisition of new responses was characterized by an increase in errors, particularly among older adults. The authors reported that these errors decreased following increased exposure to each sequence, and concluded that, among older adults, pacing restrictions wielded a greater influence on the acquisition of new sequences than on the performance of previously learned sequences.

In a related study, Perone and Baron (1983a) conducted a study comparing the effect of different time-restraints on the errors of 4 healthy older adults and 4 healthy younger adults on the acquisition and performance tasks described above. Results indicated that omission errors increased during paced sessions as a function of age, response pace, and novelty of response sequence (acquisition vs. performance). The negative effects of pacing decreased with increased exposure to temporal restraints. As a result, age differences decreased. However, older adults continued to make more errors during the acquisition component than did younger adults. Older adults' errors were particularly frequent, compared to younger adults, when the experimental protocol allowed them to correct their errors. The authors concluded that age-related differences in task performance can be reduced via increased exposure to a task.

Baron, Menich, and Perone (1983) compared the reaction times of younger and older men during a task in which increasingly short response latencies were reinforced. Participants were 7 healthy younger adults, and 7 healthy older adults. Participants were exposed to a chained reaction-time task, in which pulling a plunger started the reaction-time phase of each trial. 
During this phase, participants were cued to complete either a matching-to-sample task or an oddity task. In the matching-to-sample task, a stimulus was presented, and participants had to press the one of two available keys that corresponded to (i.e., matched) the presented stimulus. In the oddity task, however, participants were instructed to press the key that did not match the sample stimulus. Results indicated that, for both young and old, reaction times decreased as stringency of time limits increased. During both a baseline phase (in which there were no restrictions on reaction time), and a training phase (in which time restrictions were in place), younger adults generally responded faster than older adults. However, age distributions of response speed overlapped sufficiently to call reliability of findings into question. Inferential statistics were calculated. Results indicated that training produced faster reactions, that older men's responses were significantly slower than younger, and that training improved the performance of both groups, but did not reduce the age differences.

Perone and Baron (1983b) evaluated age-related preferences for paced (i.e., time-limited) and unpaced tasks in 7 healthy younger and 7 healthy older men, using a procedure similar to that just described. Following unpaced, training sessions, some participants were exposed to an "acute" pacing condition, whereas others were exposed to a "chronic" pacing condition. In the acute condition, a series of increasing (e.g., .5, 1, 2, 3 s) and decreasing (e.g., 3, 2, 1, .5 s) time limits were imposed. Time constraints in the acute condition increased or decreased following each session. Participants in the chronic condition were exposed to the same time limits. However, in the chronic condition, each time constraint was imposed for six consecutive sessions in the case of decreasing series, and for three consecutive sessions during the ascending series. Preference was indexed by the speed with which participants completed the initial link of a chained schedule of reinforcement. Increased speed in completing the initial link, and thereby 
gaining access to the terminal link was taken to indicate increased preference for the "psychological properties" of the second link (p. 165). Results indicated that, in the "acute" condition, older men made more omission errors than younger men, and that these differences were greatest in the acquisition component. In addition, older adults' initial link speeds varied as function of reinforcement rate. Initial link speeds were greater with familiar (performance) sequences. There was a weaker, but still positive relation between preference and reinforcer rate for younger men. In the "chronic" condition, a slower increase in speed demands led to a weaker relation between initial link speed and reinforcer rate in older adults. This pattern was also seen in the performance of younger men. The most efficient responding for men from both age groups occurred when correction of errors was not permitted. The authors concluded that operant paradigms provide an effective means of investigating age-related preferences for the requirements of tasks. Based on the increased speed with which older participants completed the initial link of the unpaced task (compared to the reduced speed with which they completed the link that began the paced task), the authors reported that their results support other findings that older adults prefer less rapidly paced tasks, especially when the task is novel and difficult.

Baron and Menich (1985) evaluated the effects of temporal contingencies on response speed and memory in younger and older adults. Participants were five healthy younger, and five healthy older adults. The experimental procedure involved a two-link chain. The first link involved pulling a plunger. This started the second link, a reaction-time task. Specifically, participants were instructed to hold down two telegraph keys (one with a finger of each hand). Next, a stimulus was displayed. Then, either immediately or after an interval, stimuli appeared on the left and right sides of a computer screen. At this point, subjects had to select the stimulus that matched the earlier stimulus by releasing the corresponding (i.e., left or right) key. 
Responses that occurred too early were penalized; the procedure was reset to the beginning of the chain (i.e., pulling the plunger). Initially, the choice task was unpaced. Later, however, time restrictions were placed on choices to evaluate whether such constraints have a differential effect on the behavior of younger and older subjects. Results indicated that men of both ages increased their response speed as time limits became increasingly stringent, and that both age groups had slower response speeds with increased delays in the presentation of stimuli for matching. The authors interpreted these decreased speeds as an indication that the increased intervals between presentation of target stimuli and matching stimuli tapped memory (i.e., decreased response speeds reflected a retrieval process). In addition, men of both ages improved with training, so the performance of younger men remained superior to that of older men.

Fisher and Noll (1996) investigated age-associated differences in sensitivity to reinforcement frequency. Subjects were 4 healthy older adults, and 5 healthy younger adults. Subjects participated in 8 10-minute sessions involving 3 different concurrent VI-VI schedules. The authors evaluated age differences in matching of response rates on each of two telegraph keys to the reinforcement rates determined by the distinct schedules associated with each key. They hypothesized that healthy older adults would come to match response rates to reinforcer rates more slowly than younger adults, but would do so as well as younger participants with increased exposure to contingencies. Results were generally consistent with this hypothesis. Although there were no statistically significant group differences in acquisition of matching, a trend emerged: older adults took longer to acquire matching but were more sensitive to reinforcement following acquisition.

Finally, Plaud, Muench Plaud, and von Duvillard (1999) investigated whether older adults exhibited the phenomenon of behavioral momentum. Behavioral momentum is said to 
occur when behavior that was previously reinforced continues to occur after environmental contingencies change (Plaud et al.). Participants were 15 older adults from the community ( 9 women, 6 men) without health problems that would limit their participation. During a baseline period, participants were exposed to concurrent schedules (i.e., participants could press 1 of 2 keys at any time). Initially, pressing the key associated with a green disk was rewarded with a larger magnitude of reinforcer (i.e., tokens) than pressing the key associated with a red disk; both keys operated on a VI 45-s schedule. After 6 sessions, the authors changed contingencies to either a multiple VI 30-s, multiple VI 60-s, multiple VT 30-s, multiple VT 60-s, or extinction (EXT; i.e., cessation of reinforcement). Results were consistent with behavioral-momentum theory. Specifically, participants continued to bias responses in favor of the key associated with the green disk, following change in the contingencies of reinforcement. Compared to responding of younger adults in another study by Plaud, Gaither, and Lawrence (1997, as cited in Plaud et al., 1999), older adults demonstrated lower absolute levels of responding. However, older adults' responding was biased to the green key more than that of younger adults. Plaud et al. wrote that the older participants were, therefore, "very sensitive to the changing environmental contingencies. .." (p. 174). However, increased susceptibility to behavioral momentum effects suggests a decreased sensitivity to changes in contingencies (i.e., continuing to respond according to obsolete contingencies).

Taken together, the results of published operant studies comparing healthy older and younger adults suggest that, compared to healthy younger adults, healthy older adults have slower reaction times, and that these differences cannot be improved, relative to the performance of younger adults, through training (Baron \& Menich, 1985; Baron, Menich, \& Perone, 1983). In addition, findings indicate that older adults make more errors in operant sequencing tasks that 
require increased degrees of speed, compared to younger adults. However, these differences can be reduced via training (Perone \& Baron, 1982, 1983). Furthermore, older and younger adults appear to be equally sensitive to contingencies of reinforcement when responding on concurrent schedules (Fisher \& Noll, 1996). However, compared to younger adults, older adults have been demonstrated to be more susceptible to effects of behavioral momentum, and therefore might adapt to changes in contingencies more slowly than younger adults (Plaud et al., 1999).

Operant research with individuals with cognitive impairment. A number of human operant studies have been conducted with individuals with cognitive impairment. For example, several studies have evaluated operant learning in individuals with mental retardation (MR; Headrick, 1963; Orlando \& Bijou, 1960; Repp \& Slack, 1977; Spradlin, 1962; Wiesler, Hanson, Chamberlain, \& Thompson, 1988). The results of these studies are mixed, but generally indicate that individuals with MR can respond to changes in contingencies of reinforcement. It should be noted that children with IQs ranging from 23 to 64 have demonstrated responding comparable to that seen among individuals without retardation and nonhuman subjects (Orlando \& Bijou) and that extinction effects have been observed among "severely mentally retarded" individuals (Spradlin).

Researchers have also compared the operant tasks performances of individuals with brain injury to the performances of controls (Schlund, Pace, \& McGready, 2001; Schlund, 2002a, 2002b). Findings suggested that individuals with acquired brain injury (to regions unspecified by the authors) can discriminate between contingencies of reinforcement, but are less sensitive than controls to changes in these contingencies. Thus, the performance of individuals with cognitive impairment on operant tasks indicates that the behavior of at least some members of this rather heterogeneous population is amenable to change via the manipulation of contingencies of 
reinforcement.

Operant research with older adults with dementia. A small number of operant studies have been conducted with older adults with dementia, to evaluate the degree to which learning can occur in the context of neurological disease or insult. In one such study, Ankus and Quarrington (1972) evaluated operant learning in older adults with memory disorders. The authors reported that their first experiment was based on Mackay's observation (1965, as cited in Ankus \& Quarrington, 1972) that individuals with memory disorders fail to respond to behavioral interventions, even when reward rate is quite high. They attempted to further evaluate the extent to which increasing ratio requirements by various degrees affected the amount of schedule control demonstrated by 12 "elderly patients" ( 6 men) from the residential unit of a Canadian psychiatric hospital. The experimental procedure involved a preliminary phase, in which subjects were taught to pull a lever without receiving reinforcement. Participants were then assigned to 1 of 2 groups. In the first group, participants were exposed to large, abrupt increases in an FR schedule; this was referred to as the "Pennies + High Pacing" (PHP) condition. Specifically, the number of responses required for reinforcement increased by 5 whenever the schedule changed. The second group was the "Pennies + Low Pacing (PLP)" condition, in which participants were exposed to relatively small increases in an FR schedule; the number of responses required for reinforcement in successive schedules increased by a magnitude of 3. (Note that in these studies "pacing" refers to the magnitude of ratio increases, and not to the presence or absence of time limits.) Analyses revealed that groups were equivalent in terms of baseline response rates. No significant effects of pacing, sex, or schedules were observed. However, when data for male and female subjects were analyzed separately, a significant main effect of schedules (i.e., PLP vs. PHP) was observed. Specifically, women's 
rates of responding were significantly higher under the PHP schedule than under the PLP schedule. In order to evaluate the role of reinforcers in this gender difference, the authors conducted a second study.

In their second experiment, Ankus and Quarrington (1972) included a new sample ( $N=$ 24; 12 male) of older adults with memory disorders. A procedure similar to the one from the first experiment was used. However, in this study, participants were provided with the fluid of their choice as a reinforcer, instead of pennies. Participants were assigned to either the Fluid + High Pacing (FHP) condition or the Fluid + Low Pacing (FLP) condition. Changes in ratio magnitude were larger for the FHP group, compared to those used in the first experiment (FR 5, FR 10, FR 20, FR 40). Participants were fluid deprived and fed salty foods prior to experimental sessions. Analyses indicated that there were no significant differences between FHP and FLP groups in responding during baseline. Response rate increased as the number of responses required to obtain reinforcement increased. Data for male and female subjects were then evaluated separately. Analyses revealed a significant effect of schedules (i.e., changes in FR magnitude was associated with orderly changes in responding) in male, but not female, subjects. Thus, unlike the results of the first experiment, these results indicated that the responding of male, but not female, participants came under experimental control.

In a related study, Burgess, Wearden, Cox, and Rae (1992) evaluated operant conditioning in subjects with dementia. Experiment 1 involved 3 female residents on a psychogeriatric ward (ages 74, 83, and 84 years). The consequence for lever-pressing was an auditory and visual stimulus, consisting of tones and lines. During Condition A (acquisition phase), in which participants were prompted to press the lever, 2 of the 3 women met acquisition criteria (20 unprompted responses in 5 minutes). These two women were exposed to Condition 
B, which consisted of a series of FI schedules (i.e., FI 5-s, FI 15-s, FI 10-s). Response rate was highest during the FI 15-s schedule, lower in the FI 10-s schedule, and lowest in the FI 5-s schedule. Thus, the responding of these participants was sensitive to the manipulation of contingencies of reinforcement, as defined by Madden et al. (1998), although they did not conform to the schedule-typical patterns of responding observed in infrahuman subjects.

In Experiment 2, the authors lowered the criteria for moving to Condition B. This time, participants were 2 men with dementia from a psychogeriatric ward (aged 54, and 61 years). In Condition A (acquisition component), only 10 unprompted responses in 5 min were required to move on to Condition B. Both participants met this criterion. In Condition B, these participants were exposed to a combination of FI 10-s, FI 17.5-s, and FI 25-s schedules. In this condition, participants demonstrated sensitivity. Whereas one participant's responding increased in direct proportion to the length of the FI parameter, the other participant's responding decreased with the length of the interval. Both participants showed the "scalloped" patterns of responding associated with FI intervals, in which response rates are low toward the beginning of each interval and sharply increase toward the end of each interval (i.e., just prior to reinforcement).

In sum, only two laboratory studies of operant learning in older adults with dementia have been published. The results of these investigations suggest that the responding of older adults with dementia can be sensitive to changes in contingencies of reinforcement (Ankus \& Quarrington, 1972; Burgess et al., 1992). These studies represent an important beginning to a nascent area of operant research. However, methodological problems limit their contributions. For example, the studies discussed above evaluated operant learning in individuals with dementia arising from varied (and at times, poorly characterized) etiologies. Ankus and 
Quarrington vaguely described their subjects as "memory-disordered," and Burgess et al. reported that their subjects had diagnoses ranging from "Organic Brain Syndrome as a result of alcohol abuse" and "senile dementia" (p. 221-222) to "brain damage as a result of alcohol abuse" and "vascular dementia of subcortical origin" (p. 229). Thus, within each of these studies there was considerable ambiguity or heterogeneity of diagnoses. Although knowledge of the impact of a range of neurological disease processes on operant behavior is important, at this early stage of research development it is more appropriate to focus investigations on individuals with relatively similar pathologies because very little is currently known regarding the impact of specific neurological diseases on sensitivity to changes in contingencies of reinforcement. Given that dementias arising from distinct etiologies can have idiosyncratic impacts on particular brain regions--and, in turn, on particular aspects of behavior--the failure of these studies of operant behavior to replicate findings in a population with a unitary diagnosis and underlying neuropathology precludes researchers from drawing conclusions regarding the abilities of individuals with a particular brain disease to respond to contingencies of reinforcement.

In addition, neither of the two studies published in this domain used appropriate criteria to establish stable responding before changing conditions. Ankus and Quarrington did not report using any stability criteria, and Burgess et al. simply required that subjects emit a specified number of unprompted responses within a 5-min period before moving beyond the acquisition phase. (This criterion is problematic because it specifies a number of required responses but fails to include any index of stability of responding.) Thus, the reader cannot evaluate if betweencondition changes in response rates reported by the authors was due to experimental manipulation or chance.

Furthermore, the consequences delivered to reinforce behavior in the Burgess et al. paper 
were of questionable reinforcing value. Specifically, the authors reported using an auditory and visual stimulus (tones and lines) to reinforce behavior; no rationale was provided for the use of these consequences. The authors also provided subjects with numerous prompts (e.g., "Make the music come back, please," p. 224) throughout the experiment. Thus, it is not clear to what degree responding was under the control of the programmed consequences (i.e., tones and lines) or the experimenters' instructions. Similarly, in their first experiment, Ankus and Quarrington attempted to use pennies as reinforcers. Their failure to demonstrate experimental control in male subjects might have been due to their use of a weak reinforcer. Taken together, these methodological flaws seriously limit the ability of researchers to make claims regarding the extent to which operant learning can occur in older adults with dementia.

To advance this area of research, the present study further evaluated operant learning among older adults with probable AD, using a button-pressing task. Learning was assessed in terms of sensitivity to changes in contingencies of reinforcement. Increased knowledge regarding sensitivity within a well-described sample of older adults with AD could assist clinicians in the design of behavioral interventions for agitation by explicating the extent to which operant learning can occur within a diagnostically circumscribed, yet large and growing population. To test the limits of learning in this population, this study evaluated the sensitivity of participants' responding to transitions from FR to FI schedules, and from FI to EXT schedules. Comparable tests of sensitivity have been described by other authors (Madden, Chase, \& Joyce, 1998), and have been used with human participants in the past (Hayes et al., 1986). In addition, this preparation provided the opportunity to observe whether the findings of Plaud et al. (1999) regarding increased behavioral momentum were evident in another sample.

In the present study, the duration of experimental conditions was established via formal 
stability criteria, and higher-value coins (i.e., nickels) were used as reinforcers. Furthermore, older and younger adults without neurological conditions served as controls. These methodological refinements to previous studies in this domain were made to: (a) increase the likelihood of obtaining experimental control over subjects' responding; (b) provide a normative context in which to interpret the responding of older adults with $\mathrm{AD}$; and (c) increase the confidence that can be placed in findings in this new and growing area of research. As indicated above, it is not currently clear whether, or how much, $\mathrm{AD}$ interferes with operant behavior. However, an extensive literature has developed describing the cortical structures and processes that facilitate reward-oriented learning, and the neurotransmitter dopamine is believed to play a significant role in operant conditioning (Schultz, 2002; Thompson, 2000). Studies of human AD patients have reported changes in dopamine D2 receptors in various brain regions, including the temporal lobes (Joyce, Myers, \& Gurevich, 1998; Kemppainen et al., 2003), the striatum (Kemppainen, Ruottinen, Nagren, \& Rinne, 2000; Rinne, Sahlberg, \& Ruottinen, 1998) and the olfactory bulb (Loopuijt \& Sebens, 1990). Thus, it appeared likely that operant conditioning would be affected by $\mathrm{AD}$. Because this study was largely exploratory, however, no formal hypotheses were made regarding responding.

\section{Method}

\section{Participants}

Participants with AD were four older adults ( 1 male; all Caucasian; mean age $=79.5$; range $=68-86)$ who had been diagnosed with probable $\mathrm{AD}$ by a neurologist, according to the National Institute of Neurological and Communicative Disorders and Stroke-Alzheimer's Disease and Related Disorders Association (NINCDS-ADRDA) criteria:

Dementia established by clinical examination and documented by the Mini-Mental Test 
[i.e., Mini-Mental State Examination (MMSE; Folstein, Folstein, \& McHugh, 1975)], Blessed Dementia Scale [Blessed, Tomlinson, \& Roth, 1968], or some similar examination, and confirmed by neuropsychological tests; deficits in two or more areas of cognition; progressive worsening of memory and other cognitive functions; no disturbance of consciousness; onset between ages 45-90, most often after 65; absence of systemic disorders or other brain diseases that in and of themselves could account for the progressive deficits in memory and cognition. (McKhann, Drachman, Folstein, Katzman, Price, \& Stadlan, 1984)

See Appendix A for additional information regarding the NINCDS-ADRDA criteria for diagnosis of probable $\mathrm{AD}$ (Mckhann et al.).

Participants with $\mathrm{AD}$ (were recruited from the Memory Disorders Clinic of the West Virginia University School of Medicine, from nursing homes in the Morgantown area, and from the community. Participants were informed that they would have the opportunity to earn money by participating in a study investigating learning in older adults. Exclusionary criteria for older adults with $\mathrm{AD}$ were: (a) sensory or other physical deficits that could interfere with participation (e.g., severe, uncorrected visual or auditory impairment, paralysis); (b) psychiatric diagnoses of schizophrenia, or bipolar disorder; (c) other neurological illnesses, including Parkinson's disease, Huntington's disease, Pick's disease; and (d) dementia due to vascular insult or substance abuse. The experimenter obtained informed, written consent from participants' surrogate decisionmakers. Participants with AD provided written assent. Younger comparison participants $(n=5 ; 4$ males; 3 Caucasian, 1 Black, 1 Biracial; mean age $=23.4$; range $=20-30$ ) were recruited from undergraduate psychology courses, and older comparison participants $(n=4 ; 0$ males; all Caucasian; mean age $=74.25$; range $=70-81$ ) were recruited from senior centers and via signs 
placed in a public library. Exclusionary criteria for control subjects were: (a) sensory or other physical deficits that could interfere with participation; (b) any psychiatric diagnoses or disorders; (c) psychotropic medications; and (d) neurological illness or injury. Whereas none of the younger controls were taking prescribed medications, all of the older adults in the present study were taking between 2 and 14 different medications.

\section{Setting}

Experimental sessions with older adults (both AD and controls) were conducted either in their homes or in a laboratory in the Life Sciences Building at West Virginia University. Sessions with younger adults were all conducted in the Life Sciences Building. Measures

After obtaining consent and assent, the experimenter conducted a standardized clinical interview (see Appendix B) to screen for symptoms of psychopathology, and administered the neuropsychological battery recommended by the Consortium to Establish a Registry for Alzheimer's Disease (CERAD; Morris et al., 1989) to provide descriptive data regarding participants' cognitive functioning. Descriptions of the neuropsychological tests that comprise the CERAD battery are presented below.

Animal Naming. Subjects were asked to name as many animals as they could within 1 min as a test of verbal fluency. This task has been used extensively to evaluate the verbal fluency of individuals with dementia whose abstract thinking abilities are too severe to permit the use of other such tests (Lezak, 1995).

Boston Naming Test. The Boston Naming Test (BNT; Kaplan, Goodglass, \& Weintraub, 1978,1983 ) is a 60 -item test of the ability to name pictured objects (Spreen \& Strauss, 1998). Subjects are presented with a series of line drawings of objects, which they are asked to name. 
These range from the common (e.g., tree) to the relatively rare (e.g., protractor). If they fail to name the stimuli spontaneously, they are provided with a stimulus cue (e.g., "It measures angles"). If subjects still cannot name the object, a phonemic cue is provided (e.g., "pro-"). The BNT has been shown to have good 6-month response consistency when used with individuals with AD (Henderson, Mack, Freed, Kemper, \& Anderson, 1990). In the CERAD battery, a 15item version of the test is used (Morris et al., 1989). According to Lezak (1995), this version is sensitive to the presence and severity of dementia.

Mini-Mental State Examination. The Mini-Mental State Examination (MMSE; Folstein, et al., 1975) is a brief, standardized screen of cognitive functioning. It contains 12 items evaluating orientation, attention, language ability, praxis, visuospatial ability, and memory. The MMSE yields a maximum score of 30; individuals without cognitive impairment generally score between 24 and 30. The MMSE has high 24-hr test-retest reliability (Folstein et al.), and is sensitive to progressive decline associated with degenerative dementia (Morris et al., 1989).

Word List Memory, Recall, and Recognition. The CERAD Word List Memory (WLM) task tests for 10 printed words (Morris et al., 1989). The subject reads the list of words out loud, three times, in three different orders. Recall is tested after each of these trials; maximum score is 30. There is also a delayed-recall component, which is followed by a recognition component (target words and foils are presented and subjects are asked to identify words from the original list). The maximum score on each of these tests is 10 .

Constructional Praxis. The CERAD Constructional Praxis (CP) task requires subjects to copy four simple line drawings (circle, diamond, rectangles, cube; Morris et al., 1989).

15-Item Geriatric Depression Scale. The 15-Item Geriatric Depression Scale (GDS; Sheikh \& Yesavage, 1986) is an abbreviated version of the 30 -item screening tool for depression 
(Yesavage et al., 1983). The 15-item GDS utilizes a clinical cut-off of either 6 or 7, and has been shown to correlate well with the original 30-item version (Sheik \& Yesavage).

Bristol Activities of Daily Living Scale. The Bristol Activities of Daily Living Scale (BADLS; Bucks, Ashworth, Wilcock, \& Siegfried, 1996) is a 20-item measure of adaptive functioning in older adults with cognitive impairment. The scale is completed by a caregiver. Each item measures an individual's functioning in a different behavioral domain during the preceding two weeks. Higher scores are associated with greater impairment.

\section{Apparatus}

Subjects were seated in front of a table containing the experimental console, which consisted of a wooden apparatus housing a coin-delivery system (a modified Coinco brand vending machine coin mechanism), two backlit buttons, and a basket into which reinforcers were delivered. Only 1 of the 2 buttons was active during the study; the other was covered with a sheet of black paper. This apparatus was connected to a computer that controlled the delivery of reinforcers (i.e., nickels). A program written in Visual Basic v 6.0 recorded all events that occurred during experimental sessions, including the time at which responses occur (relative to the beginning of the experimental session), the beginning and end of each reinforcement cycle, response rate, and inter-reinforcer interval (IRI; i.e., the amount of time elapsed between delivery of reinforcers). In addition, a small, locked, opaque cash box with a coin slot in the lid was placed on the table in front of subjects.

\section{Procedure}

Experimental sessions began when subjects were cued that they could begin button pressing (see below for details) and the backlit button on the experimental console was illuminated. Sessions ended when the light behind the button was turned off. 
Reinforcement. Button pressing was reinforced by the delivery of nickels (5-cent coins) in two conditions according to an FR schedule or an FI schedule. Button pressing was not reinforced in subsequent conditions that arranged an extinction schedule (EXT). The reinforcement cycle began with a tone. At this point the light behind the button was turned off, and the button was deactivated. Approximately $1 \mathrm{~s}$ later, the coin mechanism dispensed a nickel in the basket next to the console. Following coin delivery, the tone continued until participants placed the coin in the coin box. The experimenter signaled the deposit of coins to the computer by left-clicking a button on the screen. This terminated the tone and illuminated the button, signaling the end of the reinforcement cycle and the beginning of the next trial.

Preliminary training. Subjects were trained to respond on an increasingly lean series of FR schedules (beginning with FR 1). Training took place in 3-min blocks. To begin training, the experimenter told subjects:

"At times during this study you can earn nickels by pressing this button [Experimenter points to button]. You can keep all of the nickels that you earn. Each time you earn a nickel, you must place it in the box [Experimenter points to cash box]. You do not have to press the button; you can start pressing the button or stop pressing the button whenever you want. I cannot speak to you until this part of the study is over. Also, you can get up and walk around if you like, but please stay in the room until the study is over. Do you have any questions? [Experimenter addresses questions.] If you would like to, you may begin pressing the button now. Please remember to put any coins that you earn in the box."

If participants did not begin pressing the button independently within $1 \mathrm{~min}$, the experimenter manually placed their hands on the manipulandum, and completed a response. In addition, verbal 
prompts (e.g., "Please press the button.") were provided to increase the likelihood that subjects contacted the programmed contingencies. These prompts were faded out as responding stabilized. Each time a nickel was earned, the experimenter observed participants to ensure that they placed it in the cash box. If participants failed to do so, the experimenter instructed them to "Please place the nickel in the box," while pointing to the nickel and then the cash box. The experimenter responded to subjects' questions by stating: "I cannot answer questions until the study is over.” During preliminary training the backlit button was lit, as described above.

The experimenter gradually increased the number of responses required for reinforcement to occur, with the goal of obtaining a mean inter-reinforcement interval (IRI; i.e., amount of time that elapses between the delivery of two consecutive reinforcers) of approximately $5 \mathrm{~s}$ by the time responding stabilized. Responding was considered stable when response rates during three consecutive 3-min blocks did not differ from the mean of those blocks by more than $20 \%$.

Test conditions. Following preliminary training, subjects were exposed to three sequential experimental conditions: an FR, an FI, and an EXT condition. Each condition was comprised of a series of 3-min blocks. Distinct stimuli were associated with the FR and FI conditions. Upon transition to the FI condition, a small yellow light adjacent to the backlit button was illuminated. This light remained illuminated throughout the FI condition (including during reinforcement intervals), and the backlit button continued to function as before (i.e., it continued to be turned off during reinforcement intervals). The yellow light that was added during the FI condition is a departure from other studies that have evaluated sensitivity. This signaled change was implemented for two purposes. The first was to more closely emulate the conditions under which behavior management plans for older adults with dementia are executed in the "real world." In actual clinical practice, the individual with dementia is typically notified when contingencies of 
reinforcement change (e.g., "Mrs. Jones, the nursing staff will no longer give you attention when you scream. If you would like attention, you must speak in a normal, quiet voice."). Second, the change from FR to FI was signaled to increase the likelihood of obtaining sensitive responding in older adults with $\mathrm{AD}$ to a subtle change in contingencies. The stimulus conditions for the EXT condition were the same as in the FI condition; no cue was deemed necessary to facilitate sensitive responding to the gross change in contingencies (i.e., cessation of reinforcement delivery) involved in the transition from FI to EXT. The computer controlled transitions between all conditions, based on the stability of participants' responding.

The ratio value that was in effect when participants' responding stabilized during preliminary training was used as the FR parameter during the FR condition. Thus, if the FR magnitude under which a subject's responding stabilized during training was an FR 10 schedule, this ratio was used in the FR condition of the baseline phase. During the FR condition, responding was considered stable when response rate during three consecutive blocks did not differ from the mean of those blocks by more than $20 \%$. The FI condition began when responding stabilized.

The FI condition was also conducted in 3-min blocks. The length of the fixed interval during this condition was programmed to be equal to the mean inter-reinforcement interval obtained by subjects during stable responding in the FR condition. For example, a subject who obtained a mean IRI of $10 \mathrm{~s}$ during the FR condition was exposed to an FI 10-s schedule. Stability criteria implemented in the FI condition were the same as those used in the FR condition. Once responding stabilized, the schedule was changed to EXT.

The EXT condition was conducted in 3-min blocks, and continued until the mean response rate over two consecutive blocks fell to $25 \%$ or less of the mean response rate during 
stable blocks of the FI condition.

Retention test. Memory deficits are a core feature of AD. To evaluate the effect of the disease on the retention of acquired responses, participants were re-exposed to the EXT condition 24 to 48 hours following the end of the initial button-pressing session. This was referred to as the retention condition (RET). Reductions in the number of blocks to extinction criteria were to be taken as an indication of retention of learning from the initial session. The experimenter read the following instructions:

"The machine works just like it did last time. I cannot speak to you until this part of the study is over. You do not have to press the button; you can start pressing the button or stop pressing the button whenever you want."

This test was conducted under stimulus conditions that were identical to their prior exposure to EXT, and with the same extinction criteria. Any reduction in the number of sessions it took for responding to meet extinction criteria compared to the EXT component of the initial button pressing session were interpreted to reflect retention (i.e., memory) of the initial session.

In sum, the experimental procedure was conducted in two or three sessions. During the initial meeting, the experimenter administered psychosocial and cognitive measures. Either immediately following, or within one week of this assessment, the initial button-pressing session was completed. The retention test was administered 24 to $48 \mathrm{hrs}$ following the end of the initial button-pressing session.

Results

Psychological Measures

Participants' performances on the CERAD neuropsychological battery are presented in Table 2. Scores of younger adults and older adult controls were comparable. As would be 
expected, the performances of older adults with $\mathrm{AD}$ on these tests were generally lower than those of other participants, although memory for true negatives on the recognition portion of the CERAD WLM was roughly equivalent across groups. It should also be noted that, given his very high level of education (23 years), participant AD31 is probably more impaired, relative to his baseline, than his $z$ scores imply. Beyond providing separate norms for individuals with less than 12 years or 12 or more years of education, the CERAD norms (Welsh et al., 1994) do not account for level of education.

Two of the older participants with AD and one college student scored above the clinical cutoff on the GDS. None of the older controls did so. None of the control participants exhibited any deficits in adaptive functioning, as measured by the Bristol ADL Scale, whereas all of the participants with $\mathrm{AD}$ exhibited difficulties in activities of daily living.

\section{Operant Task}

The following sections detail participants' button-pressing performances under preliminary training, FR, FI, EXT, and RET conditions. Each participant's data first are presented along with those of his or her peers (i.e., those of other college students, cognitively intact older adults, or older adults with AD). Next, observed commonalities and differences among groups' performances are presented. All response rates reported refer to the mean number of responses for the 3-min blocks that met stability criteria, within each condition. Data concerning the parameters obtained by participants during FR and FI schedules, and mean response rates are presented in Table 3.

In addition to narrative and graphic descriptions of each participant's performance, a quantitative index of the sensitivity of participants' responding to changes in contingencies of reinforcement was calculated. A similar index was employed by Hayes et al. (1986). In the 
present study, the sensitivity index was calculated by dividing the mean response rate during the stable blocks of each condition by the mean response rate during the stable blocks of the condition that preceded it. Thus, the index describing participants' sensitivity to the change from an FR schedule to an FI schedule was determined by dividing the mean stable response rate during the FI condition by the mean stable response rate in the FR condition. Similarly, the mean stable response rate during the EXT condition was divided by the mean stable response rate during the FI condition to describe numerically participants' sensitivity to the transition from an FI schedule to EXT. The sensitivity index number is more intuitively understood when converted to a figure reflecting the percent change in response rate obtained by participants during stable responding after each change in contingencies. For this reason, the data regarding sensitivity of participants' performances are discussed in terms of percent change. Please see Table 4 for further details regarding sensitivity indices and percent change in participants' responding across conditions. Cumulative records of each participant's responding are displayed in Appendixes F through R.

\section{College Students}

Participant Y10's responding accelerated rapidly during preliminary training (see Figure 1). His responding stabilized after 5 blocks had elapsed. He obtained a mean response rate of 1148.67. His responding was maintained at this high rate through the FR 35 condition. However, it decreased rapidly upon transition to an FI 5.26-s schedule, clearly demonstrating sensitivity to this change in schedules. In fact, transition from the FR to FI schedules resulted in a 96.34\% reduction in response rate. Y10's responding met stability criteria after 10 blocks in the FI condition, during which the mean response rate was 44.33. Responding met EXT criteria after four blocks during this condition. Participant Y10's responding during the RET condition is an 
example of the phenomenon known as spontaneous recovery. Spontaneous recovery is said to occur when a behavior that has been extinguished during a previous learning session reappears following a delay (Martin \& Pear, 1999). This is a well-documented phenomenon in basic research (Martin \& Pear). Spontaneous recovery is evident when one compares Y10's response rate during the initial block of the RET condition to his response rate during the final blocks of the EXT condition. During the RET condition, responding met criteria in three blocks.

During preliminary training, Y22's responding stabilized in four blocks at a mean rate of 1270.33 (see Figure 2). Although responding decreased immediately following transition to the FR 39 schedule, it increased sharply in the second block of the condition, and stabilized after four blocks at a mean rate of 1459.33. Participant Y22's responding was highly sensitive to the transition from the FR to the FI 5.37-s schedule. This change in rate is reflected in slope of the response function, which becomes almost horizontal shortly following the change to the FI condition. Responding dropped precipitously (by 97.88\%) during the FI condition, and stabilized in ten blocks. No EXT or RET data was available for Y22 due to experimenter error.

Participant Y21's responding followed a pattern that was similar to, yet less extreme, than the responding of the Y10 and Y22 (see Figure 3). It increased during preliminary training, and stabilized after four blocks at the more moderate rate of 558.67. Y21 obtained an FR parameter of 19 , during which responding was maintained at a mean rate of 575 . It decreased upon transition to an FI 5.86-s schedule, and stabilized at a mean rate of 286, after four blocks. Although Y21's responding was decreasing slightly prior to the transition to the FI condition, the discrepancy between the steepness of the slopes of the two functions displayed in the FR and FI conditions of Figure 5 suggests that the rapid decrease during the FI condition is due to the experimental manipulation, rather than spurious factors. Indeed, Y21 responding reduced by just 
over $50 \%$ following transition to the FI condition. During the EXT condition, responding increased slightly, and then slowly decreased in an almost linear fashion, meeting criteria EXT in five blocks. Participant Y21 demonstrated spontaneous recovery of previously extinguished responding at the beginning of the RET condition. EXT criteria were met in three blocks during RET.

Participant Y17 obtained stable responding after four blocks of preliminary training, at a mean rate of 647.67 (see Figure 4). Responding remained at this rate through the FR 18 condition (mean rate $=627.67)$ and was insensitive to transition to an FI 5.10-s schedule. Responding was highly sensitive to the transition to EXT; it rapidly decreased by almost 93\%, and stabilized at a mean of 41. In addition, participant Y17 demonstrated spontaneous recovery of responding during RET. EXT criteria were met in two blocks in both EXT and RET conditions.

Y18's response rate increased during preliminary training, and stabilized at a mean of 662.67 in three blocks. Response rate increased somewhat during the FR 18 condition (mean rate $=759.67$ ) and continued at approximately the same rate during the FI 4.38-s condition (mean rate $=784)$. Responding decreased immediately upon transition to EXT, and met EXT criteria in five blocks. Y18 demonstrated spontaneous recovery of responding in the RET condition, during which EXT criteria were met in two blocks. See Figure 5 for further details.

Summary. Of the 5 college students who participated, 3 (Y10, Y21, and Y22) exhibited responding that was sensitive to the transition from FR to FI schedules (see Table 4 for details). The responding of each of the four college students who completed the EXT condition was sensitive to the transition to EXT. During RET, all college students demonstrated spontaneous recovery of previously extinguished responding. In all four cases, participants' responding met 
EXT criteria at least as rapidly during RET as during EXT.

\section{Older Controls}

Participant OA27's response rate increased during the initial portion of preliminary training (see Figure 6), and stabilized after 4 blocks, at a stable mean of 527.66. It remained near this rate for the 3 blocks of the FR 15 condition (mean rate $=513.33$ ) and the first block of the FI 5.26-s condition. From the second block of the FI condition onward, however, responding decreased sharply, and stabilized after six blocks at a rate of 66.33 , resulting in an $87.08 \%$ reduction in response rate, and thus a high degree of sensitivity to this transition. During RET, participant OA27 demonstrated spontaneous recovery of previously extinguished responding. Although participant OA27's responding met EXT criteria in three blocks during the EXT condition, it met criteria in ten blocks during RET.

Participant OA13's response rate varied widely during the initial blocks of preliminary training (see Figure 7) and met stability criteria after six blocks (mean rate $=366.33$ ). The FR 10 component began while response rate was decreasing. Responding continued to decrease in a linear fashion during the FR condition, and stabilized at a mean rate of 234.67. A similar pattern was observed at the beginning of the FI 7.05-s condition, which began with a slight increase in responding, followed by stabilization at a lower rate (mean rate of stable blocks $=135.67)$. This change in schedules resulted in a $42.19 \%$ decrease in response rate. During RET, participant OA13 demonstrated spontaneous recovery of responding that had been extinguished during the previous EXT session. EXT criteria were met in three blocks during the initial button-pressing session and in two blocks during RET.

Participant OA20's responding was characterized by a gradual, almost linear, increase in rate across preliminary training, FR 7, and FI 7.17-s conditions, resulting in mean stable response 
rates of 151.67, 185.67, and 274, respectively (see Figure 8). Indeed, this trend makes it particularly difficult to determine whether the change in OA20's response rate is due to the change in contingencies of reinforcement or a spurious factor (e.g., increasing dexterity resulting from prolonged exposure to the task). Participant OA20's responding resulted in a $47.75 \%$ increase in response rate upon transition from FR to FI schedules. Response rate decreased immediately and markedly upon transition to EXT; EXT criteria were met in three blocks. During RET, participant OA20 demonstrated spontaneous recovery of responding. EXT criteria were met in 12 blocks during RET.

Participant OA26's responding increased at the beginning of preliminary training, and stabilized in four blocks (see Figure 9). Response rate failed to change substantially between preliminary training, FR 8, and FI 5.29-s schedules (mean rates $=262.33,274.33,289.67$, respectively). This participant's responding met EXT criteria within two blocks of transition to EXT, as well as during the RET condition, which contained another example of spontaneous recovery.

Summary. Only one of the 4 cognitively intact older controls that participated exhibited responding that was highly sensitive (see Table 4) to the transition from FR to FI schedules (OA27). The responding of participants OA13 and OA20 might have been sensitive to this change in contingencies. However, in these participants' cases, schedules of reinforcement were changed when response rate was either increasing or decreasing. As a result, firm conclusions regarding the sensitivity of these participants' responding to the transition from FR to FI schedules cannot be drawn. Participant OA20's responding increased somewhat following transition from the FR to the FI schedule, although a decrease in response rate would have been a more efficient response to this change in schedules. The responding of each older control 
participant was sensitive to the change from FI to EXT schedules. During the RET condition, all older controls demonstrated spontaneous recovery of responding that had been extinguished during the EXT condition of the previous session. Of these 4 participants, 2 exhibited responding that took longer to meet stability criteria during RET than during EXT.

Older Adults with Probable AD

Participant AD23's responding increased during preliminary training, and stabilized at a mean rate of 132.33. Responding continued to increase throughout the FR 5 condition, and met stability criteria at a mean rate of 170 (see Figure 10). Responding began to decrease immediately upon transition to the FI 5.46-s schedule, during which it stabilized at a mean rate of 165. This resulted in a change in stable response rate of less than 3\%. Thus, this participant's responding was affected initially by this change in contingencies, but did not change to the extent that one might have predicted, given the distinct demands of the experimental conditions schedules. Participant AD23's responding decreased precipitously following transition to EXT, yielding a $94.24 \%$ change in response rate. Responding meting EXT criteria in three blocks, at a mean response rate of 9.5. Participant AD23 failed to demonstrate spontaneous recovery of responding during the RET condition; responding during the initial block of RET was not notably higher than it was at the end of EXT. EXT criteria were met in two blocks during the RET condition.

Participant AD30's response rate increased throughout preliminary training, stabilizing at a mean rate of 424 (see Figure 11). Responding stabilized in three blocks during the FR 15 condition, at a mean rate of 662.67. This rate of responding was maintained during the FI 4.02-s schedule. Responding stabilized in three blocks, yielding a mean rate of 673.33. AD30's response rate decreased rapidly upon transition to EXT. During this condition, responding 
stabilized in 7 blocks, at a mean rate of 0.0. Participant AD30 failed to demonstrate spontaneous recovery of responding during the RET condition. During RET, AD30's responding met EXT criteria in two blocks.

Participant AD31's response rate increased steadily during preliminary training, and stabilized in 4 blocks at a mean rate of 302.33 (see Figure 12). During the FR 9 condition, responding was maintained at a mean rate of 320.33 , and stabilized within 3 blocks. Responding decreased significantly (i.e., by $28.82 \%$ ) during the FI-5.06 s condition. It stabilized in 3 blocks at a mean rate of 228. During EXT, participant AD31's responding decreased gradually to a mean rate of 43, meeting EXT criteria in 6 blocks. Participant AD31 failed to demonstrate spontaneous recovery of previously extinguished responding during the RET condition, in which EXT criteria were met in two blocks.

Participant AD28 was unable to acquire the button-pressing task sufficiently well to complete the experimental procedure. Her initial attempt to acquire the task was interrupted when she became agitated, reported that she did not feel well, and returned to her room in the nursing facility where she was evaluated. After taking a break of more than one hour, AD28 reported that she felt better, and returned to attempt the task again. Although she was able to complete the preliminary training condition (mean rate $=137.33$ ), she suddenly became confused regarding the sequence of behaviors required to obtain nickels. This resulted in a long pause in responding (see Appendix S for a cumulative record of responding). Eventually, the experimenter reminded AD28 how to obtain nickels, and this resulted in temporary reacquisition of the task. Soon afterwards, however, AD28 became confused again, and was unable to reacquire the task in spite of verbal prompting by the experimenter.

Summary. Of the 4 participants with probable AD, 3 acquired the operant task. Of these 
3, 2 (AD23, AD31) demonstrated responding that was somewhat sensitive (see Table 4) to the transition from an FR to an FI schedule. Although participant AD23 did not exhibit a significant change in response rate between FR and FI conditions, the trend of her responding reversed immediately upon transition to the FI schedule, indicating some sensitivity to this change in contingencies. All 3 of the participants who acquired the task were sensitive to the transition from an FI schedule to EXT. None of the older adults with probable AD demonstrated spontaneous recovery of the responding that had been extinguished during the previous EXT condition. The responding of all three of the older participants with $\mathrm{AD}$ who completed the protocol met EXT criteria more rapidly during RET than during the initial EXT condition. Only one of the 4 participants with $\mathrm{AD}, \mathrm{AD} 28$, was unable to acquire the task.

\section{Between-Groups Comparisons}

The present study was conducted using a single-subject design. No inferential statistical analyses were performed. Nonetheless, two groups of cognitively intact participants (i.e., younger controls, older controls) were included to provide a normative point of reference to which the performances of older adults with $\mathrm{AD}$ could be compared via visual inspection of data. In this section, the performances of each of these groups are compared and contrasted. Although these comparisons cannot yield firm conclusions regarding group differences, they provide preliminary data that could be used to guide future investigations.

The most obvious difference between the performances of younger and older participants was the difference in mean response rates obtained by each group during the stable blocks of the FR condition. In all but one case (AD30), the mean response rates of younger controls during the stable blocks of the FR condition were higher than older adults with and without AD. As discussed above, stability criteria were met during the FR condition by 3 of the 4 participants 
with $\mathrm{AD}$. Of these individuals, two exhibited mean stable response rates that were higher than those of most older controls.

Other findings of interest are in the area of sensitivity. Of the 5 participants who were college students, 3 (Y10, Y21, Y22) exhibited decreases in response rate in excess of 50 percent upon transition from FR to FI schedules. The responding of each of these participants decreased rapidly upon transition to the FI condition. By the second block of the FI condition, these 3 younger participants exhibited decreases in responding to as little as $12 \%, 54 \%$, and $8 \%$ of the mean response rate during the FR condition. Only 1 (OA27) of the 4 older controls demonstrated a change in response rate that was greater than $50 \%$, although the responding of 2 others (OA13, OA20) approached this criterion. In addition, whereas decreases in younger participants' response rates occurred almost immediately following transition to the FI condition, OA27 responding decreased and met the stability criterion far more gradually; prolonged exposure to the FI schedule was required before stability was obtained.

The responding of participants in all groups was highly sensitive to the transition from FI to EXT. Younger controls and older adults with AD tended to exhibit the highest levels of sensitivity to EXT (as measured by percent change in response rates between FI and EXT conditions). No substantial between-groups differences were evident in the number of blocks required for participants' responding to meet EXT criteria during the initial button-pressing session; the responding of older controls met EXT criteria more rapidly than that of younger adults and older adults with $\mathrm{AD}$ following transition from FI to EXT schedules.

Although all college students and older controls demonstrated spontaneous recovery of previously extinguished responding during the RET condition, no older adults with $\mathrm{AD}$ who completed the EXT and RET conditions exhibited this pattern of responding. In addition, none of 
the four younger participants who completed the EXT and RET tasks exhibited responding that took longer to meet extinction criteria during the RET condition than during the EXT condition. Of the four older controls that completed these conditions, however, two (OA20 and OA27) exhibited increased time to extinction. None of the three older adults with AD who completed the protocol exhibited responding that took longer to reach EXT criteria during RET than during their initial exposure to the EXT schedule.

\section{Discussion}

The present study utilized a button-pressing task to evaluate the extent to which operant conditioning can occur in older adults with probable $\mathrm{AD}$, and contained a number of methodological refinements to previous studies in this domain. These included the use of formal stability criteria, higher-value reinforcers (i.e., nickels), four older adults with a presumably uniform underlying neuropathology (i.e., AD), and older and younger adults without neurological conditions (i.e., controls).

The most important findings of the present study are that: (a) the older adults with mild to moderate $\mathrm{AD}$ acquired a novel task when reinforcement was provided according to $\mathrm{FR}$ schedules; and (b) this behavior was maintained on both FR and FI schedules. The performances of the participants with AD are consistent with those described by Ankus and Quarrington (1972), in which some participants with memory disorders were able to acquire a lever-pulling task, and exhibited responding (in Experiment 2) that was sensitive to changes in FR schedules. The present findings also echo those of Burgess et al. (1992), in which participants with dementia were sensitive to changes in FI schedules. Thus, these findings provide further, more robust evidence of the possibility of operant learning among older adults with dementia. In addition, the use of the NINCDS-ADRDA criteria (McKhann et al., 1984) for inclusion in the 
present study enables researchers, for the first time, to make confident statements about the potential for operant conditioning among older adults with probable AD.

The primary implication of the finding that operant learning can occur among older adults with mild to moderate $\mathrm{AD}$ is that interventions based on operant principles may well be effective with this population in the natural environment. The findings of the present study contradict the "therapeutic nihilism" that one commonly encounters when working in clinical settings with older adults with AD (Haley, 1983, p. 20). Indeed, psychologists, nursing staff, and caregivers (both formal and informal) of older adults with AD should be made aware of these findings, which along with the results of previous studies could dispel--or at least qualify-commonly held beliefs regarding the inability of older adults with AD to respond to contingencies of reinforcement. Furthermore, the positive results of applied studies of behavioral interventions to manage maladaptive behavior in this population, combined with this growing basic research literature, amount to very good news given the problems related to the use of medication in older adults (e.g., sedation, increased risk of falls, increased cognitive impairment, medication interactions). That is not to say that medications should not be used to manage behavior problems in older adults with dementia. Indeed, there is reason to believe that medications such as donepezil can reduce the rate of cognitive decline (Klatte, Scharre, Nagaraja, Davis, \& Beversdorf, 2003; Matthews, Korbey, Wilkinson, \& Rowden, 2000), and that antipsychotic medications such as risperidone and olanzapine can help reduce agitation in this population (Brodaty, Ames, \& Snowdon, 2003; Street, Clark, \& Kadam, 2001). Nonetheless, research indicates an increased incidence of negative medication side effects in older adults (Rumsey, 1989) including cognitive impairment, delirium (Mulsant, Pollock, Kirshner, Shen, Dodge, \& Ganguli, 2003), and an increased risk of neuroleptic-induced parkinsonism (Mamo, 
Sweet, \& Mulsant, 2002). Empirical data supports the use of interventions to reduce behavioral disturbances based on operant principles (Allen-Burge et al., 1999; Burgio \& Fisher, 2000; Fisher \& Carstensen, 1990; Kasl-Godley \& Gatz, 2000) as a legitimate alternative, or at least a potential adjunct, to pharmacological interventions toward the same end.

A second important finding of the present study is that the older adults with mild to moderate $\mathrm{AD}$ discriminated between schedules of reinforcement that were highly dissimilar (i.e., FI and EXT schedules), even when the transition between them was unsignaled. In addition, results indicate that the responding of some individuals with mild to moderate $\mathrm{AD}$ might be sensitive to finer-grained changes in contingencies (i.e., from an FR schedule to a yoked FI schedule), as was seen in the case of participant AD31. This finding suggests that clinicians should consider making changes in contingencies of reinforcement highly salient. One means of doing this is to make the new contingencies implemented to change a behavior highly discrepant from those that previously maintained that behavior. This can result in some rather counterintuitive interventions. For example, if a resident in a long-term care facility is exhibiting disruptive vocalization that is being maintained on a very thin FR schedule, staff might opt to increase the richness of the reinforcement schedule prior to implementing an extinction intervention. Doing so would increase the salience of the change in contingencies (relative to the salience of the transition from a thin FR schedule to extinction), which could increase the likelihood of sensitivity to extinction. Indeed, this sort of manipulation has been used repeatedly within the animal (Moreland, Stalling, \& Walker, 1983; Stalling, Moreland, Merrill, \& Scotti, 1981) and human operant literature. In studies with human participants with developmental disabilities, a continuous reinforcement (CRF) condition has been inserted between an intermittent schedule of reinforcement condition and extinction, leading to decreased resistance 
to extinction (Higbee, Carr, \& Patel, 2002; Schmid, 1988). The inserted CRF schedule is commonly referred to as an interpolated $C R F$ condition.

Another intriguing finding is the failure of one participant, with $\mathrm{AD}$ (AD28) and relatively high neuropsychological test scores, to acquire the novel task sufficiently well to permit an evaluation of sensitivity. Interestingly, the cognitive test scores of AD28 were not generally any lower than those of other participants with AD. Her performances on the MMSE and the 15-item BNT, for example, were actually higher than those of two participants with $\mathrm{AD}$ who were able to acquire the task and complete the experimental protocol. Indeed, her MMSE score was 26 . This is above 23 , the cutoff commonly used in clinical settings when screening for dementia. This finding indicates that an individual who performs well on some common neuropsychological measures might be unable to learn a basic operant task. By extension, that individual may have an impaired ability to learn from the environmental consequences of his or her behavior, which could manifest in problematic behaviors, including perseverative and generally maladaptive behavior (e.g., repetitive questioning, repeatedly "bothering" others in spite of consequences that would ordinarily be punishing). This finding underscores the importance of informing formal and informal caregivers that an individual with $\mathrm{AD}$ who appears relatively cognitively intact in many domains (e.g., converses adequately, dresses neatly) will not necessarily be able to respond normally in all domains (e.g., when acquiring novel tasks that require sensitivity to contingencies of reinforcement), especially if she is somewhat agitated, as AD28 was prior to discontinuing the present study.

Given that tests commonly administered to assess cognitive status in older adults with memory impairment may not be valuable measures for estimating the amenability of an individual's behavior to operant conditioning, the best test of this ability may simply be the 
administration of an operant task. Though such tests are not commonly administered in clinical settings they might be useful with $\mathrm{AD}$ patients who exhibit behavioral difficulties--whether they reside at home with family caregivers, or in long-term care settings--to evaluate the extent to which their behavior can come under experimental control. This evaluation could involve exposure to various schedules, to determine those that best facilitate the acquisition and maintenance of a response by the patient in question. In addition, preferred items (e.g., edibles, music) could be dispensed in lieu of nickels in a clinical setting that does not require the consistency called for in a research context. Indeed, a stimulus preference assessment in which a range of stimuli are presented to patients and orienting responses (e.g., looking at stimuli, gesturing toward stimuli) are observed to determine the relative reinforcing values of stimuli (see Fisher \& Buchanan, 2000), would be an excellent complement to this sort of evaluation.

Another important finding of the present study was that 3 of the 5 younger participants exhibited high sensitivity to the transition from FR to FI, but such sensitivity was almost absent among older adults with and without AD. Indeed, only one participant in each of the latter two groups demonstrated sensitivity to this transition. These preliminary findings indicate that, relative to younger individuals, both cognitively intact and cognitively impaired older adults might have a reduced capacity to make fine discriminations (e.g., between yoked FI and FR schedules), compared to grosser discriminations (e.g., between FI and EXT schedules). This finding conflicts somewhat with that reported in prior studies. Fisher and Noll's (1996) investigation of age-associated differences in sensitivity to reinforcement frequency on concurrent schedules yielded no statistically significant age-related differences in acquisition of matching following increased exposure to a task. That study did report a trend toward age-related differences in acquisition and sensitivity, although it was opposite to that observed in the present 
study: older adults tended to acquire matching more slowly than younger participants, but were more sensitive to differences between concurrent VI (i.e., VI-VI) schedules following acquisition. The discrepancy between the present findings and those obtained by Fisher and Noll might have been a function of this study's use of sequential, rather than concurrent, schedules. Alternatively, the previous study's finding that older adults took longer to acquire matching (i.e., sensitivity) suggests that the present findings regarding sensitivity in older participants might have been different, had stability criteria required a greater number of blocks of stable responding (i.e., increased exposure to each condition), prior to changing conditions.

Although older participants demonstrated relatively low levels of sensitivity to the transition from FR to FI schedules, both groups of older adults showed high sensitivity to the transition from FI to EXT; responding of older controls actually met extinction criteria more rapidly than that of younger participants and participants with $\mathrm{AD}$. Thus, the behavior of older adults without neurological impairment might be prone to more rapid extinction than younger adults and older adults with $\mathrm{AD}$, and thus more sensitive to this type of schedule change. The observation of more rapid extinction of responding in older controls than in older adults with $\mathrm{AD}$ is not particularly surprising; older controls are presumed to be cognitive intact and common sense indicates that this would lead to greater sensitivity among controls. Increased sensitivity in older controls compared to younger participants, however, is somewhat perplexing. Perhaps the more rapid extinction of older controls' responding was due a longer learning history, which includes more experience with EXT schedules. Alternatively, it could be that older controls became fatigued more rapidly than younger participants, and that this fatigue was an establishing operation that increased the reinforcing value of decreasing responding, relative to that of receiving nickels. 
Another interesting finding of the present study is that the older adults with AD and younger controls took no longer to meet extinction criteria during the retention test than during the initial EXT condition and that two of the older controls (OA20, OA27) did. Although it initially might seem counterintuitive, these apparently superior performances during retention of older participants with probable AD could actually be attributed to their memory deficits. During this study's conception, it seemed that re-exposing participants (after a delay) to the final schedule of reinforcement that they experienced during their initial button-pressing session would be an effective means of investigating the impact of the memory deficit characteristic of $\mathrm{AD}$ on the retention of an operant task. However, the final schedule to which participants were exposed during the initial session was EXT. Thus, in this study, greater "retention" would be evidenced by rapid extinction of responding upon re-exposure to this contingency. Although participants with $\mathrm{AD}$ tended to demonstrate greater retention after a delay (i.e., rapid extinction) this is more parsimoniously explained by reduced memory of the task itself. That is, older adults with AD may well have shown greater retention (i.e., reached EXT criteria more rapidly) than older controls because they had forgotten either how to perform the task, or that button pressing had at one time been rewarded with nickels.

A related finding is that college students and older controls demonstrated spontaneous recovery of previously extinguished responding during the RET condition, whereas older adults with AD did not. Reduced memory for the button-pressing task was offered above as the most parsimonious explanation for the observation that older adults with $\mathrm{AD}$ tended to take less time to meet extinction criteria during the RET condition than during the EXT condition. The absence of spontaneous recovery among these participants also could be explained by this memory deficit. A noteworthy clinical implication follows from this finding: extinction-based 
interventions might be more easily implemented among older adults with AD than among individuals without a memory disorder. Although applied research is needed in this domain to investigate this, our finding suggests that, individuals with intact memories might be more likely than individuals with $\mathrm{AD}$ to exhibit spontaneous recovery of a previously extinguished, maladaptive response. It should be noted, however, that the present study observed this phenomenon in the context of a novel experimental task. Applied extinction-based interventions are more likely to target problematic behaviors with a much longer history of reinforcement than the task used in the present study. It is not currently clear whether these findings regarding spontaneous recovery will generalize to the findings of applied extinction-based interventions.

Younger participants in the present study tended to perform the experimental task more rapidly (i.e., with higher response rates) than older controls. This is consistent with the findings of operant studies comparing the performances of younger adults and cognitively intact older adults (Baron and Menich 1985; Perone and Baron, 1983b; Plaud et al., 1999). Interestingly, older adults with $\mathrm{AD}$ also tended to exhibit higher response rates than older controls. The latter finding might have been due to frontal/executive dysfunction that is commonly observed in older adults with AD (APA, 1994), which can produce motoric perseveration and dyscontrol.

\section{Limitations and Recommendations for Future Research}

One of the limitations of the present study was the inclusion of both male and female participants. Although there are no substantial gender differences in the human operant literature, an all-male or all-female sample would have allowed for more compelling between-groups comparisons. The problem is particularly apparent when we compare older and younger participants; the vast majority of older participants are female, whereas most of the younger participants are male. Future studies might limit their sample to one sex. 
A second limitation of this study is the fact that younger participants were not taking any prescribed medications and older participants were, in general, taking a number of such medicines. We cannot readily determine the extent to which medications impacted performance on psychological measures or the operant task. In a related area, each of the older participants with $\mathrm{AD}$ were taking medications to slow the progression of $\mathrm{AD}$, including donepezil, galantamine, and memantine. It is not currently clear if, or to what extent, such medications impact operant learning. Initial studies indicate that cholinesterase inhibitors such as tacrine and pyridistigmine bromide impact performance on operant tasks (Ohta, Matsumoto, Shimizu, \& Watanabe, 1994; Shih et al., 1991).

The present study improved upon the methodologies of earlier investigations (e.g., Ankus \& Quarrington, 1972; Burgess et al., 1992) through the use of stability criteria. However, the stability criteria used in this study should have included criteria regarding schedule transitions following response rates that are increasing or decreasing. Because these were absent in the present study, there were instances in which experimental control was not evident following schedule changes. Thus, the stability criteria used in the present study were an improvement to past studies, but are not entirely unproblematic. Future studies in this area should include stability criteria that preclude schedule changes when response rates are increasing or decreasing in a fashion consistent with what would be expected during the subsequent schedule.

The present study investigated sensitivity to two changes in conditions: from FR to FI schedules, and from FI to EXT schedules. Future studies investigating sensitivity to changes in schedules of reinforcement among older adults with or without dementia might investigate sensitivity to transitions among a broader range of schedules. It would be particularly interesting to evaluate sensitivity to the transition from an FR schedule to a differential reinforcement of low 
rates of behavior schedule (DRL). There is a more substantial difference in the consequences of continuing to respond at a steady rate after a schedule changes from FR to DRL than there is in continuing stable responding after a schedule changes from an FR schedule to a yoked FI schedule (as was the case in the present study). Although the responding of older adults with and without dementia was less sensitive to the transition from FR to FI than that of younger adults, the responding of all three of these groups might have been equally sensitive to the transition from FR to DRL schedules.

In addition, clinicians working in long-term care would benefit from further research investigating the extent to which the behavior of older adults with $\mathrm{AD}$ can be maintained on thin schedules of reinforcement. Nursing staff members are confronted with a potentially overwhelming number of tasks each day, and behavioral interventions that rely on very high rates of reinforcement by staff are unlikely to be implemented correctly. Increased knowledge regarding the maintenance of behavior on thinner schedules could lead to interventions that are less demanding on staff, and more likely to be implemented as designed. In addition, the multiple demands facing staff members in these facilities could impede their ability to reliably implement an intervention based on a strict FI schedule. Researchers are encouraged to investigate the amenability of the responding of older adults with dementia to control via VI schedules. The use of VI schedules in interventions in long-term care settings would provide more temporal flexibility for staff members facing an overwhelming number of tasks. In addition, applied studies investigating the prevalence of spontaneous recovery of previously extinguished problem behaviors in older adults with $\mathrm{AD}$ could provide useful information regarding the extent to which geriatric clinicians should be concerned about this potentially problematic phenomenon.

The present study examined the behavior of four older adults with AD. Multiple 
replications of this study are encouraged to evaluate the reliability of the results obtained. In addition, systematic examination of schedule control with older participants with other welldefined dementias and different severities of impairment would be helpful in determining the range of populations in which interventions based upon operant conditioning are appropriate. Furthermore, studies examining schedule control in individuals with dementias with multiple etiologies would be useful. Findings in this area would be particularly useful for treatment planning in long-term care facilities, where conditions such as $\mathrm{AD}$ frequently occur in the context of other disease processes (e.g., vascular changes, Parkinson's disease). Although the behavioral approach is largely ideographic, the eventual development of normative data concerning the extent to which the behavior of individuals with various neurological illnesses can be brought under control of particular schedules of reinforcement is a worthwhile goal. Such data could provide clinicians with a general idea of which schedules would most likely be effective in controlling behavior, and could help them avoid attempting interventions based on schedules of reinforcement to which individuals with particular a diagnosis (or combinations of diagnoses) are unable to respond.

In a related area, future studies of operant conditioning in older adults with $\mathrm{AD}$ might include participants with more severe degrees of cognitive impairment, as these are the individuals who are more likely to exhibit higher rates of disruptive behavior that require management or modification. One limitation of the present study was its use of participants with mild to moderate $\mathrm{AD}$, as measured by neuropsychological test performance. Nonetheless, the results of the present study have implications for researchers interested in this area of study. Specifically, it should be noted that the relatively simple task utilized in the present investigation was too complex for a participant with mild $\mathrm{AD}$ to acquire, and that an even simpler task might 
be developed for future studies to maximize the likelihood of acquisition. It will be a challenge to develop a task that is at once simple enough to permit acquisition in individuals with significant cognitive impairment, yet engaging enough to prevent attrition due to boredom; participants in the present study reported boredom on several occasions.

Although the CERAD neuropsychological battery (Morris et al, 1989) was administered to participants in the present study, a more comprehensive battery of neuropsychological measures to better elucidate the specific cognitive skills that are associated with operant task acquisition and sensitivity to transitions among similar and dissimilar schedules of reinforcement. Common sense dictates that sensitivity to changes in the environment relies in part upon attention to those changes. In addition, because sensitivity is generally measured in terms of changes in response rate, it follows that behavioral flexibility, as opposed to perseveration on obsolete contingencies, would be a necessary condition for sensitivity. Thus, the inclusion of measures of executive function including attention and behavioral flexibility would be an interesting addition to future research in this area. This would also allow for the testing of hypotheses generated above regarding the potential impact of executive dysfunction on response rates and sensitivity in operant studies.

Given the problems with interpreting the meaning of the retention data collected in the present study, it is suggested that future studies in this domain examine retention differently. Instead of exposing participants to an extinction schedule and testing for retention of extinguished responding (i.e., a lack of responding) after a delay, researchers are encouraged to test for retention of an experimental task.

Finally, additional research is needed to investigate the greater sensitivity to subtle changes in contingencies of reinforcement observed in the performances of younger participants, 
and the more rapid extinction observed among older controls. As suggested above, increasing the duration of exposure to each condition might yield more equitable results among older and younger participants, in terms of sensitivity. 


\section{References}

Allen-Burge, R., Stevens, A. B., \& Burgio, L. D. (1999). Effective behavioral interventions for decreasing dementia-related challenging behavior in nursing homes. International Journal of Geriatric Psychiatry, 14, 213-232.

Alzheimer's Association. (2002). Statistics: About Alzheimer's disease [Brochure]. Chicago: Author.

American Psychiatric Association. (1994). Diagnostic and statistical manual of mental disorders ( $4^{\text {th }}$ ed.). Washington, DC: Author.

Ankus, M., \& Quarrington, B. (1972). Operant behavior in the memory-disordered. Journal of Gerontology, 27, 500-510.

Ballard, C. G., Margallo-Lana, M., Fossey, J., Reichelt, K., Myint, P., Potkins, D., et al. (2001). A 1-year follow up study of behavioral and psychological symptoms in dementia among people in care environments. Journal of Clinical Psychiatry, 62, 631-636.

Baron, A., \& Menich, S. R. (1985). Age-related effects of temporal contingencies on response speed and memory: An operant analysis. Journal of Gerontology, 40, 60-70.

Baron, A., Menich, S. R., \& Perone, M. (1983). Reaction times of younger and older men and temporal contingencies of reinforcement. Journal of the Experimental Analysis of Behavior, 40, 275-287.

Baron, A., \& Perone, M. (1982). The place of the human subject in the operant laboratory. The Behavior Analyst, 5, 143-158.

Birchmore, T., \& Clague, S. (1983). A behavioral approach to reduce shouting. Nursing Times, $37-39$. 
Bird, M., Alexopoulos, P., \& Adamowicz, J. (1995). Success and failure in five case studies: Use of cued recall to ameliorate behavior problems in senile dementia. International Journal of Geriatric Psychiatry, 10, 305-311.

Blessed, G., Tomlinson, B. E., Roth, M. (1968). The association between quantitative measures of dementia and of senile change in the cerebral grey matter of elderly subjects. British Journal of Psychiatry, 114, 797-811.

Block, C., Boczkowski, J. A., Hansen, N., \& Vanderbeck, M. (1987). Nursing home consultation: Difficult residents and frustrated staff. Gerontologist, 27, 443-446.

Boehm, S., Whall, A. L., Cosgrove, K. L., Locke, J. D., \& Schlenk, E. A. (1995). Behavioral analysis and nursing interventions for reducing disruptive behaviors of patients with dementia. Journal of Applied Nursing Research, 8, 118-122.

Bourgeois, M. S., Schulz, R., \& Burgio, L. (1996). Interventions for caregivers of patients with Alzheimer's disease: A review and analysis of content, process, and outcomes. International Journal of Aging and Human Development, 43, 35-92.

Brodaty, H., Ames, D., Snowdon, J. (2003). A randomized placebo-controlled trial of risperidone for the treatment of aggression, agitation, and psychosis of dementia. Journal of Clinical Psychiatry, 64, 134-143.

Bucks, R. S., Ashworth, D. L., Wilcock, G. K., \& Siegfried, K. (1996). Assessment of activities of daily living in dementia: Development of the Bristol Activities of Daily Living Scale. Age and Ageing, 25, 113-120.

Burgess, I. S., Wearden, J. H., Cox, T., \& Rae, M. (1992). Operant conditioning with subjects suffering from dementia. Behavioral Psychotherapy, 20, 219-237. 
Burgio, L. D., \& Fisher, S. E. (2000). Application of psychosocial interventions for treating behavioral and psychological symptoms of dementia. International Psychogeriatrics, 12(Suppl. 1), 351-358.

Buskist, W. F., Miller, Jr., \& Bennett, R. H. (1980). Fixed-interval performances in humans: Sensitivity to temporal parameters when food is the reinforcer. Psychological Record, 30, $111-121$

Chandler, J. D., \& Chandler, J. E. (1988). The prevalence of neuropsychiatric disorders in a nursing home population. Journal of Geriatric Psychology and Neurology, 1, 71.

Cohen-Mansfield, J., \& Billig, N. (1986). Agitated behaviors in the elderly: I. A conceptual review. Journal of the American Geriatrics Society, 34, 711-721.

Cohen-Mansfield, J., Werner, P., Watson, V., \& Pasis, S. (1995). Agitation among elderly persons at adult day-care centers: the experiences of relatives and staff members. International Psychogeriatrics, 7, 447-458.

Dube, W. V., \& McIlvane, W. J. (2002). Quantitative assessments of sensitivity to reinforcement contingencies in mental retardation. American Journal on Mental Retardation, 107, $136-145$.

Fisher, J. E., \& Buchanan, J.A. (November, 2000). Distraction based intervention for aggression in dementia patients. Paper presented at the Association for the Advancement of Behavior Therapy, New Orleans.

Fisher, J. E., \& Carstensen, L. L. (1990). Behavior management of the dementias. Clinical Psychology Review, 10, 611-629.

Fisher, J. E., \& Noll, J. P. (1996). Age-associated differences in sensitivity to reinforcement frequency. Journal of Clinical Geropsychology, 2, 297-306. 
Folstein, M. F., Folstein, S. E., \& McHugh, P. R. (1975). Mini-mental state: A practical method for grading the cognitive state of patients for the clinician. Journal of Psychiatric Research, 12, 189-198.

Haley, W. E. (1983). A family-behavioral approach to the treatment of the cognitively impaired elderly. Gerontologist, 23, 18-20.

Hayes, S. C., Brownstein, A. J., Haas, J. R., \& Greenway, D. E. (1986a). Instructions, multiple schedules, and extinction: Distinguishing rule-governed from schedule-controlled behavior. Journal of the Experimental Analysis of Behavior, 46, 137-147.

Hayes, S. C., Brownstein, A. J., Zettle, R. D., Rosenfarb, I., \& Korn, Z. (1986b). Rule-governed behavior and sensitivity to changing consequences of responding. Journal of the Experimental Analysis of Behavior, 45, 237-256.

Headrick, M. W. (1963). Effects of instructions and initial reinforcement on fixed-interval behavior in retardates. American Journal of Mental Deficiency, 68, 425-432.

Henderson, V. W., Mack, W., Freed, D. M., Kemper, D., \& Anderson, E. S. (1990). Naming consistency in Alzheimer's disease. Brain and Language, 39, 530-538.

Herrnstein, R. J., (1961). Relative and absolute strength of response as a function of frequency of reinforcement. Journal of the Experimental Analysis of Behavior, 4, 267-272.

Herrnstein, R. J. (1970). On the law of effect. Journal of the Experimental Analysis of Behavior, 13, 243-266.

Higbee, T. S., Carr, J. E., \& Patel, M. R. (2002). The effects of interpolated reinforcement on resistance to extinction in children diagnosed with autism: A preliminary investigation. Research in Developmental Disabilities, 23, 61-78. 
Hussian, R. A. (1982). Stimulus control in the modification of problematic behavior in elderly institutionalized patients. International Journal of Behavioral Geriatrics, 1, 33-42.

Hussian, R. A. (1988). Modification of behaviors in dementia via stimulus manipulation. Clinical Gerontologist, 8, 37-43.

Hussian, R. A., \& Brown, D. C. (1987). Use of two-dimensional grid patterns to limit hazardous ambulation in demented patients. Journal of Gerontology, 42, 558-560.

Iwata, B. A., Pace, G. M., Dorsey, M. F., Zarcone, J. R., Vollmer, T. R., Smith, R. G., et al. (1994). The functions of self-injurious behavior: An experimental-epidemiological analysis. Journal of Applied Behavior Analysis, 27, 215-240.

Joyce, J. M., Myers, A. J., \& Gurevich, E. (1998). Dopamine D2 receptor bands in normal human temporal cortex are absent in Alzheimer's disease. Brain Research, 784, 7-17.

Kaplan, E. F., Goodglass, H., \& Weintraub, S. (1978). Boston Naming Test (Experimental ed.). Boston: Kaplan \& Goodglass.

Kaplan, E. F., Goodglass, H., \& Weintraub, S. (1983). Boston Naming Test $\left(2^{\text {nd }}\right.$ ed.). Philadelphia: Lea \& Febiger.

Kaplan, H. I., \& Sadock, B. J. (1998). Kaplan and Sadock's synopsis of psychiatry (8 ${ }^{\text {th }}$ ed.). Baltimore, MD: Williams \& Wilkins.

Kasl-Godley, J., \& Gatz, M. (2000). Psychosocial interventions for individuals with dementia: An integration of theory, therapy, and a clinical understanding of dementia. Clinical Psychology Review, 20, 755-782.

Kemppainen, N., Laine, M., Laakso, M. P., Kaasinen, V., Någren, K., Vahlberg, T., et al. (2003). Hippocampal dopamine D2 receptors correlate with memory functions in Alzheimer's disease. European Journal of Neuroscience, 18, 149-154. 
Kemppainen, N., Ruottinen, H., Nagren, K., \& Rinne, J. O. (2000). PET shows that striatal dopamine D1 and D2 receptors are differentially affected in AD. Neurology, 55, 205-209.

Klatte, E. T., Scharre, D. W., Nagaraja, H. N., Davis, R. A., Beversdorf, D. Q. (2003). Combination therapy of donepezil and vitamin E in Alzheimer disease. Alzheimer Disease and Associated Disorders, 17, 113-116.

Kochanek, K. D., Smith, B. L., \& Anderson, R. N. (2001). Deaths: Preliminary data for 1999. National Vital Statistics Reports, 49, 1-52. Hyattsville, Maryland: National Center for Health Statistics.

Kollins, S. H., Newland, M. C., \& Critchfield, T. S. (1997). Human sensitivity to reinforcement in operant choice: How much do consequences matter? Psychonomic Bulletin \& Review, 4, 208-220.

Labouvie-Vief, G., Hoyer, W. J., Baltes, M. M., \& Baltes, P. B. (1974). Operant analysis of intellectual behavior in old age. Human Development, 17, 259-272.

Lezak, M. D. (1995). Neuropsychological assessment ( $2^{\text {nd }}$ ed.). New York: Oxford University Press.

Loopuijt, L. D., \& Sebens, J. B. (1990). Loss of dopamine receptors in the olfactory bulb of patients with Alzheimer's disease. Brain Research, 529, 239-244.

Mackay, H. A. (1965). Operant techniques applied to disorders of the senium. Unpublished doctoral dissertation, Queen's University, Kingston, Ontario.

Madden, G. J., Chase, P. N., \& Joyce, J. H. (1998). Making sense of sensitivity in the human operant literature. The Behavior Analyst, 21, 1-12.

Mamo, D. C., Sweet, R. A., \& Mulsant, B. H. (2002). Neuroleptic-induced parkinsonism in Alzheimer's disease. Drug-induced movement disorders in the elderly [Special issue]. 
Psychiatric Annals, 32, 249-252.

Martin, G., \& Pear, J. (1999). Behavior modification: What it is and how to do it (6th ed.). Upper Saddle River, NJ: Simon \& Schuster.

Matthews, H. P., Korbey, J., Wilkinson, D. G., Rowden, J. (2000). Donepezil in Alzheimer's disease: Eighteen month results from Southampton Memory Clinic. International Journal of Geriatric Psychiatry, 15, 713-720.

Mayer, R., \& Darby, S. J. (1991). Does a mirror deter wandering in demented older people? International Journal of Geriatric Psychiatry, 6, 607-609.

McKhann, G., Drachman, D., Folstein, M., Katzman, R., Price, D., \& Stadlan, E. M. (1984). Clinical diagnosis of Alzheimer's disease: Report of the NINCDS-ADRDA Work Group under the auspices of Department of Health and Human Services Task force on Alzheimer's Disease. Neurology, 34, 939-944.

Moreland, J. W., Stalling, R. B., Walker, L. C. (1983). The effect of interpolated continuous reinforcement on persistence in extinction: A replication demonstrating reversibility. Behaviour Analysis Letters, 3, 149-156.

Morris, J. C., Heyman, A., Mohs, R. C., Hughes, J. P., van Belle, G., Fillenbaum, G., et al. (1989). The Consortium to Establish a Registry for Alzheimer's Disease (CERAD). Part I: Clinical and neuropsychological assessment of Alzheimer's disease. Neurology, 39, 1159-1165.

Mulsant, B. H., Pollock, B. G., Kirshner, M., Shen, C., Dodge, H., \& Ganguli, M. (2003). Serum anticholinergic activity in a community-based sample of older adults: Relationship with cognitive performance. Archives of General Psychiatry, 60, 98-203. 
Namazi, K. H., Rosner, T. T., \& Calkins, M. P. (1989). Visual barriers to prevent ambulatory Alzheimer's patients from exiting through an emergency door. Gerontologist, 29, 699702.

Neef, N. A., Mace, F. C., Shea, M. C., \& Shade, D. (1992). Effects of reinforcer rate and reinforcer quality on time allocation: Extensions of matching theory to educational settings. Journal of Applied Behavior Analysis, 25, 691-699.

Nelson, R. O., \& Hayes, S. C. (1986). The nature of behavioral assessment. In R. O. Nelson \& S. C. Hayes (Eds.), Conceptual foundations of behavioral assessment (pp. 3-41). New York: Guilford.

O’Hare, E., Weldon, D. T., Mantyh, P. W., Ghilardi, J. R., Finke, M. P., Kuskowski, M. A., et al. (1999). Delayed behavioral effects following intrahippocampal injection of A beta (1-42). Brain Research, 815, 1-10.

Ohta, H., Matsumoto, K., Shimizu, M., \& Watanabe, H. (1994). Paeoniflorin attenuates learning impairment of aged rats in operant brightness discrimination task. Pharmacology, Biochemistry, and Behavior, 49, 213-217.

Orlando, R., \& Bijou, S. W. (1960). Single and multiple schedules of reinforcement in developmentally retarded children. Journal of the Experimental Analysis of Behavior, 3, 339-348.

Perone, M., \& Baron, A. (1982). Age-related effects of pacing on acquisition and performances of response sequences: An operant analysis. Journal of Gerontology, 37, 443-449.

Perone, M. \& Baron, A. (1983a). Reduced age differences in omission errors after prolonged exposure to response pacing contingencies. Developmental Psychology, 19, 915-923. 
Perone, M., \& Baron, A. (1983b). Age-related preferences for paced and unpaced tasks in chained schedules of reinforcement. Experimental Aging Research, 9, 165-168.

Plaud, J. J., Gaither, G. A., \& Lawrence, J. B. (1997). Operant schedule transformations and human behavioral momentum. Journal of Behavior Therapy and Experimental Psychiatry, 28, 169-179.

Plaud, J. J., Muench Plaud, D. M., \& von Duvillard, S. P. (1999). Human behavioral momentum in a sample of older adults. Journal of General Psychology, 126, 165-175.

Repp, A. C., \& Slack, D. J. (1977). Reducing responding of retarded persons by DRO schedules following a history of low-rate responding: A comparison of ascending interval sizes. Psychological Record, 3, 581-588.

Rinne, J. O., Sahlberg, N., \& Ruotinnen, H. (1998). Striatal uptake of the dopamine reuptake ligand is reduced in Alzheimer's disease assessed by positron emission tomography. Neurology, 50, 152-156.

Rumsey, K. E. (1989). Pharmacological considerations in older adults. Journal of the American Optometric Association, 60, 520-530.

Schlund, M. W. (2002). The effects of brain injury on choice and sensitivity to remote consequences: deficits in discriminating response-consequence relations. Brain Injury, $16,347-357$.

Schlund, M. W. (2002). Effects of acquired brain injury on adaptive choice and the role of reduced sensitivity to contingencies. Brain Injury, 16, 527-535.

Schlund, M. W., Pace, G. M., \& McGready, J. (2001). Relations between decision-making deficits and discriminating contingencies following brain injury. Brain Injury, 15, 10611071. 
Schmid, T. L. (1988). A comparison of two behaviour reduction procedures: Traditional extinction alone and interpolated reinforcement followed by extinction. Journal of Mental Deficiency Research, 32, 289-300.

Schultz, W. (2002). Getting formal with dopamine and reward. Neuron, 36, 241-263.

Selkoe, D. J. (2002). Alzheimer's disease is a synaptic failure. Science, 298, 789-791.

Sheikh, J. I., \& Yesavage, J. A. (1986). Geriatric Depression Scale (GDS): Recent evidence and development of a shorter version. In J. Brink (Ed.), Clinical gerontology: A guide to assessment and intervention (pp. 165-173). New York: Haworth Press.

Shih, J. H., Liu, W. E., Lee, S. F., Lee, J. D., Ma, C., \& Lin, C.H. (1991). Acute effects of oral pyridostigmine bromide on conditioned operant performance in rats. Pharmacology, Biochemistry, and Behavior, 38, 549-553.

Shimoff, E., Matthews, B. A., \& Catania, A. C. (1986). Human operant performance: Sensitivity and pseudosensitivity to contingencies. Journal of the Experimental Analysis of Behavior, 46, 149-157.

Sourial, R., McCusker, J., Cole, M., \& Abrahamowicz, M. (2001). Agitation in demented patients in an acute care hospital: Prevalence, disruptiveness, and staff burden. International Psychogeriatrics, 13, 183-197.

Spradlin, J. E. (1962). Effects of reinforcement schedules on extinction in severely mentally retarded children. American Journal of Mental Deficiency, 66, 634-640.

Spreen, O., \& Strauss, E. (1998). A compendium of neuropsychological tests ( $2^{\text {nd }}$ ed.). New York: Oxford University Press.

Stalling, R. B., Moreland, J. W., Merrill, K. H., \& Scotti, J. (1981). Continuous reinforcement interpolated between intermittent reinforcement and extinction decreases resistance to 
extinction. Behaviour Analysis Letters, 1, 89-95.

Street, J. S., Clark, W. S., Kadam, D. L. (2001). Long-term efficacy of olanzapine in the control of psychotic and behavioral symptoms in nursing home patients with Alzheimer's dementia. International Journal of Geriatric Psychiatry, 16(Suppl. 1), S62-S70.

Swearer, J. M., Drachman, D. A., O’Donnell, B. F., \& Mitchell, A. L. (1988). Troublesome and disruptive behaviors in dementia: Relationships to diagnosis and disease severity. Journal of the American Geriatrics Society, 36, 784-790.

Thompson, R. F. (2000). The brain: A neuroscience primer ( $3^{\text {rd }}$ ed.). New York: Worth.

Welsh, K. A., Butters, N., Mohs, R. C., Beekly, D., Edland, S., Fillenbaum, G., et al. (1994). The consortium to establish a registry for Alzheimer's disease (CERAD). Part V: A normative study of the neuropsychological battery._Neurology, 44, 609-614.

Wiesler, N. A., Hanson, R. H., Chamberlain, T. P., \& Thompson, T. (1988). Stereotypic behavior of mentally retarded adults adjunctive to a positive reinforcement schedule. Research in Developmental Disabilities, 9, 393-403.

Yesavage, J. A., Brink, T. L., Rose, T. L., Lum, O., Huang, V., Adey, M. B., et al. (1983). Development and validation of a geriatric depression screening scale: A preliminary report. Journal of Psychiatric Research, 17, 37-49. 
Table 1

Demographic and Psychosocial Information

\begin{tabular}{|c|c|c|c|c|c|c|c|}
\hline & Age & Sex & Race & $\begin{array}{l}\text { Education } \\
\text { (yrs.) }\end{array}$ & Prescribed Medications & $\begin{array}{c}\text { Bristol } \\
\text { ADL } \\
\text { Scale }\end{array}$ & $\begin{array}{l}15- \\
\text { item } \\
\text { GDS }\end{array}$ \\
\hline \multicolumn{8}{|l|}{ Younger Controls } \\
\hline Y10 & 20 & Male & Caucasian & 15 & -- & 0 & 5 \\
\hline Y17 & 22 & Male & Caucasian & 16 & -- & 0 & 0 \\
\hline Y18 & 24 & Male & Biracial & 12.5 & -- & 0 & 8 \\
\hline Y21 & 30 & Female & Caucasian & 16 & -- & 0 & 3 \\
\hline Y22 & 21 & Male & Black & 15 & -- & 0 & 3 \\
\hline \multicolumn{8}{|l|}{ Older Controls } \\
\hline OA13 & 81 & Female & Caucasian & 16 & alendronate, levothyroxine & 0 & 2 \\
\hline OA20 & 70 & Female & Caucasian & 14 & enalapril, $\mathrm{HCTZ}^{*}$ & 0 & 2 \\
\hline OA26 & 75 & Female & Caucasian & 18.5 & calcitonen, enalapril, omeprazole & 0 & 1 \\
\hline OA27 & 71 & Female & Caucasian & 12 & triamterene/HCTZ, rabeprazole, raloxifene & 0 & 4 \\
\hline \multicolumn{8}{|l|}{ Probable AD } \\
\hline AD23 & 86 & Female & Caucasian & 10 & $\begin{array}{l}\text { latanoprost, HCTZ, levothyroxine, lisinopril, } \\
\text { atenolol, trazodone, tramadol, galantamine }\end{array}$ & 5 & 4 \\
\hline $\mathrm{AD} 28$ & 82 & Female & Caucasian & 16 & aspirin, galantamine hydrobromide & 8 & 13 \\
\hline AD30 & 82 & Female & Caucasian & 12 & $\begin{array}{l}\text { ASA, clopidogrel, isosorbide, } \\
\text { triamterene/HCTZ, levothyroxine, quinapril, } \\
\text { esomeprazole, celecoxib, atorvastatin, } \\
\text { albuterol/ipratroprium, fluticasone, lactulose, } \\
\text { calcitonin, donepezil } \\
\text { galantamine hydrobromide, memantine, } \\
\text { bupropion, escitalopram oxalate, digoxin, } \\
\text { metoprolol, lisinopril, atorvastatin calcium, } \\
\text { aspirin }\end{array}$ & $\geq 8 * *$ & 5 \\
\hline
\end{tabular}


Table 2

Participants' Raw and (z) Scores on CERAD Neuropsychological Tests

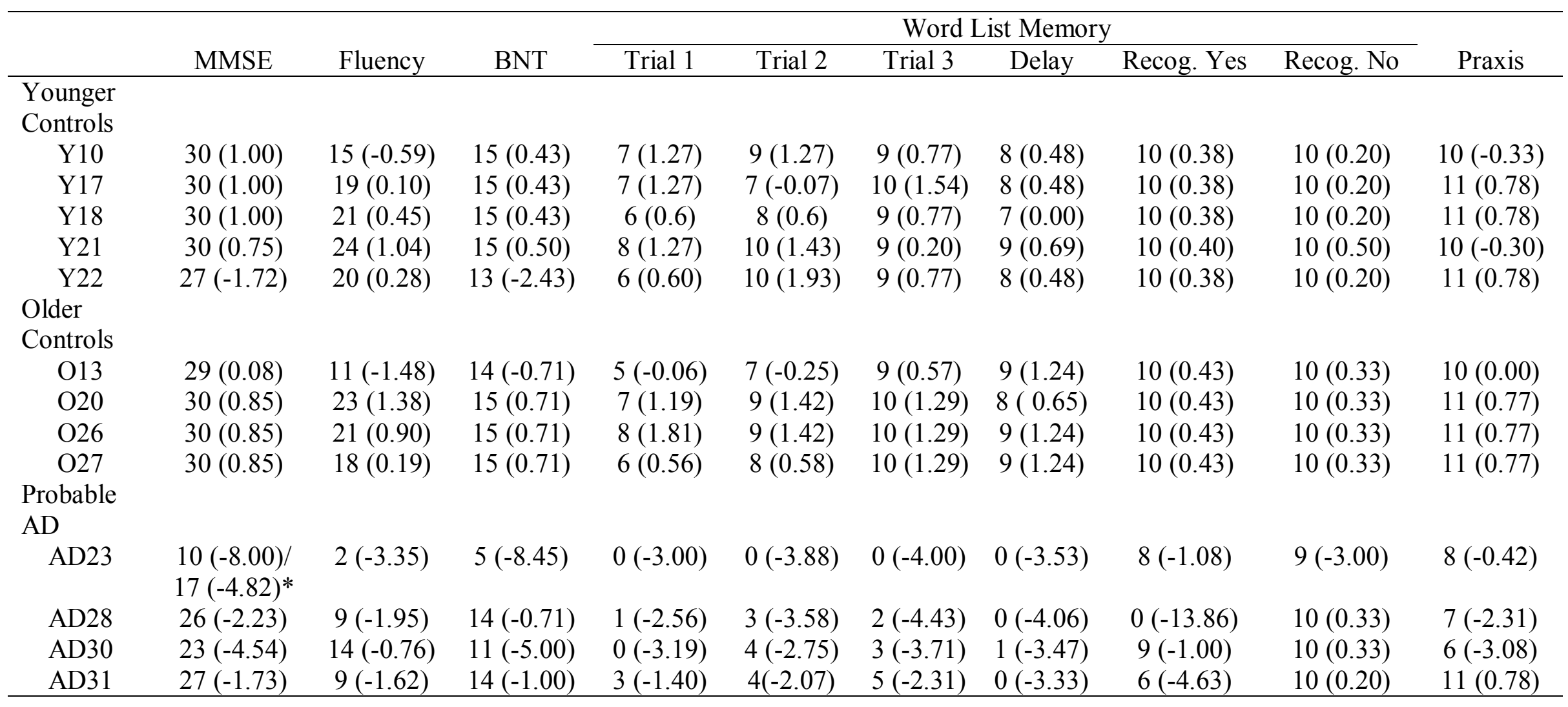

Note: Younger participants' $\mathrm{z}$ scores were calculated using normative data for adults aged 50-69 years. This is the youngest group for which CERAD has published norms (Welsh et al., 1994). *The MMSE was administered twice to participant AD23 within 24 hours; results of both administrations are presented. 
Table 3

Parameters for FR and FI Schedules, and Mean Response Rates Obtained by Participants Across Conditions

\begin{tabular}{ccccccc}
\hline & FR parameter & Mean FR & FI parameter & Mean FI & $\begin{array}{c}\text { Mean } \\
\text { EXT }\end{array}$ & $\begin{array}{c}\text { Mean } \\
\text { RET }\end{array}$ \\
\hline Younger & & & & & & \\
Controls & & & & & & \\
Y10 & 35 & 1212 & 5.26 & 44.33 & 0.5 & 0.5 \\
Y17 & 18 & 627.67 & 5.1 & 582 & 41 & 18.5 \\
Y18 & 18 & 759.67 & 4.38 & 784 & 88 & 37.5 \\
Y21 & 19 & 575 & 5.86 & 286 & 42 & 66 \\
Y22 & 39 & 1459.33 & 5.37 & 31 & -- & -- \\
Older & & & & & & \\
Controls & & & & & & \\
O13 & 10 & 234.67 & 7.05 & 135.67 & 13.5 & 6 \\
O20 & 7 & 185.67 & 7.168 & 274 & 53.5 & 62 \\
O26 & 8 & 274.33 & 5.29 & 289.67 & 55.5 & 26 \\
O27 & 15 & 513.33 & 5.26 & 66.33 & 1.5 & 0.0 \\
Probable & & & & & & \\
AD & & & & & & \\
AD23 & 5 & 170 & 5.46 & 165 & 9.5 & 0.5 \\
AD28 & 5 & -- & -- & -- & -- & -- \\
AD30 & 15 & 662.67 & 4.02 & 673.33 & 0.0 & 0.0 \\
AD31 & 9 & 320.33 & 5.06 & 228 & 23.5 & 0.5 \\
\hline
\end{tabular}


Table 4

Sensitivity Indices, Percent Change, and Retention Savings

\begin{tabular}{|c|c|c|c|c|c|}
\hline & \multicolumn{2}{|c|}{$\mathrm{FI} / \mathrm{FR}$} & \multicolumn{2}{|c|}{ EXT/FI } & \multirow[b]{2}{*}{ Retention Savingst } \\
\hline & Sensitivity* & Percent Change & Sensitivity & Percent Change & \\
\hline \multicolumn{6}{|l|}{ Younger } \\
\hline \multicolumn{6}{|l|}{ Controls } \\
\hline Y10 & 0.0366 & -96.34 & 0.0113 & -98.87 & 1 \\
\hline Y17 & 0.9272 & -7.28 & 0.0704 & -92.96 & 0 \\
\hline Y18 & 1.0320 & +3.00 & 0.1122 & -88.78 & 3 \\
\hline Y21 & 0.4974 & -50.26 & 0.1469 & -85.31 & 2 \\
\hline Y22 & 0.0212 & -97.88 & -- & -- & -- \\
\hline \multicolumn{6}{|l|}{ Older } \\
\hline \multicolumn{6}{|l|}{ Controls } \\
\hline OA13 & 0.5781 & -42.19 & 0.0995 & -90.05 & 2 \\
\hline OA20 & 1.4757 & +47.75 & 0.1953 & -80.47 & -9 \\
\hline OA26 & 1.0559 & +5.59 & 0.1916 & -80.84 & 0 \\
\hline OA27 & 0.1292 & -87.08 & 0.0226 & -61.56 & -8 \\
\hline \multicolumn{6}{|l|}{ Probable } \\
\hline $\mathrm{AD}$ & & & & & \\
\hline AD23 & 0.9706 & -2.94 & 0.0576 & -94.24 & 1 \\
\hline $\mathrm{AD} 28$ & -- & -- & -- & -- & -- \\
\hline AD30 & 1.0161 & +1.61 & 0.0000 & -100.00 & 5 \\
\hline AD31 & 0.7118 & -28.82 & 0.1031 & -99.90 & 4 \\
\hline
\end{tabular}

Note: *Sensitivity indices were calculated by dividing the mean response rate across blocks of stable responding in one condition by the mean response rates across blocks of stable responding in the condition that preceded it. $t$ Retention savings reflects the number of blocks to meet extinction criteria during initial button pressing session, minus the number of blocks to meet extinction criteria during the retention test; negative values denote a greater number of blocks to extinction during retention test than during initial session. 
Figure 1. Response rates of participant Y10 during initial and retention sessions.

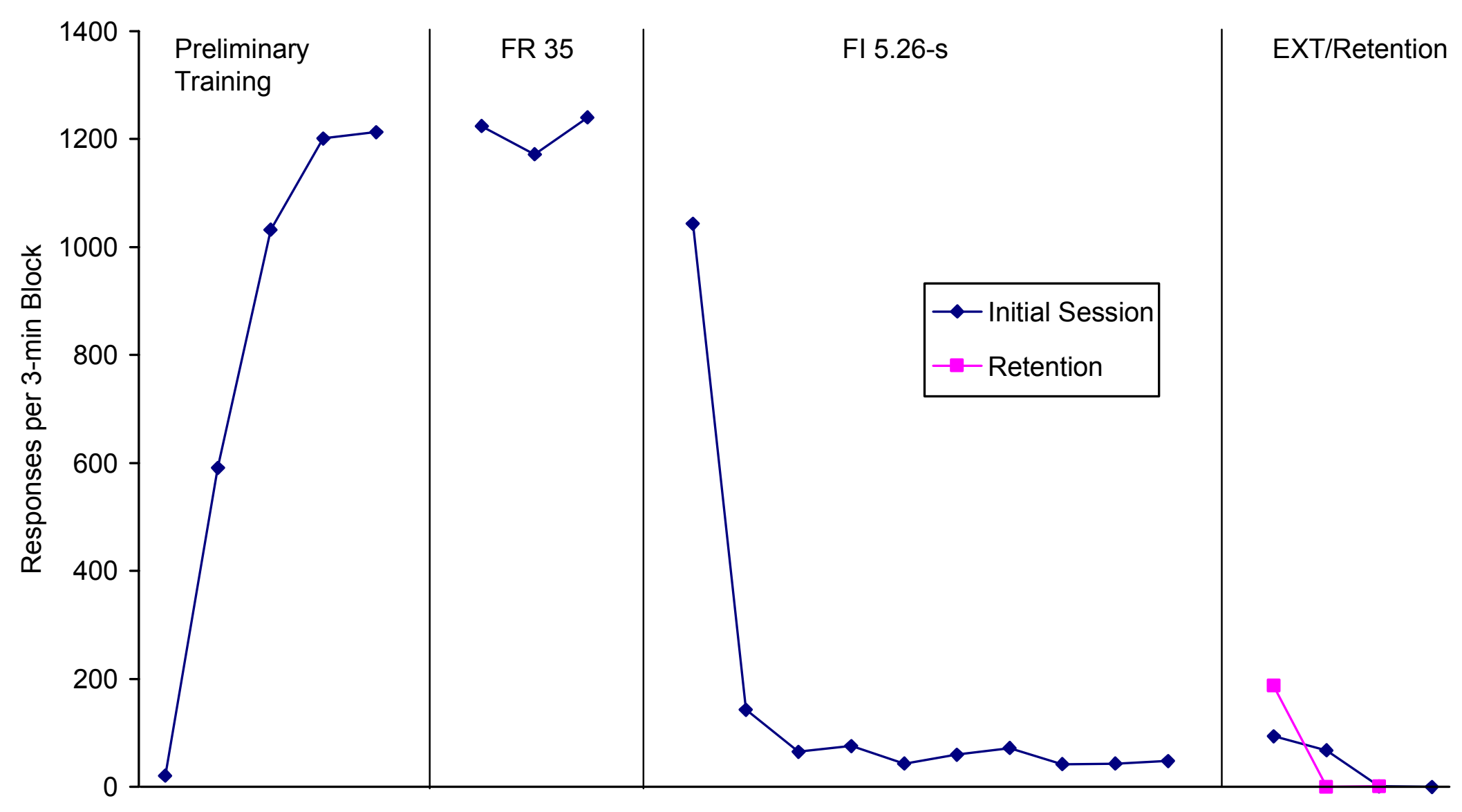


Figure 2. Response rates of participant Y22 during initial session.

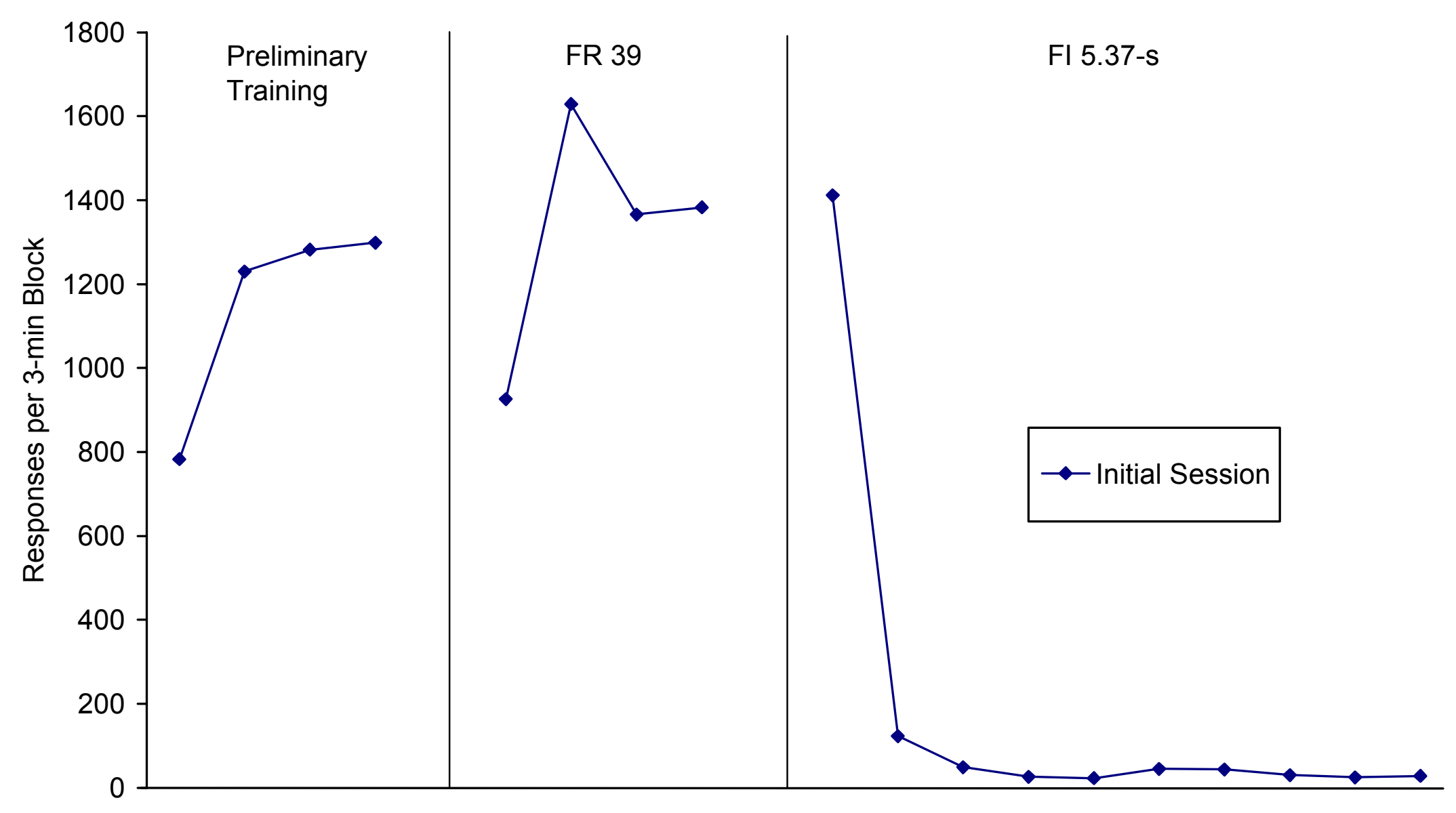


Figure 3. Response rates of participant Y21 during initial and retention sessions.

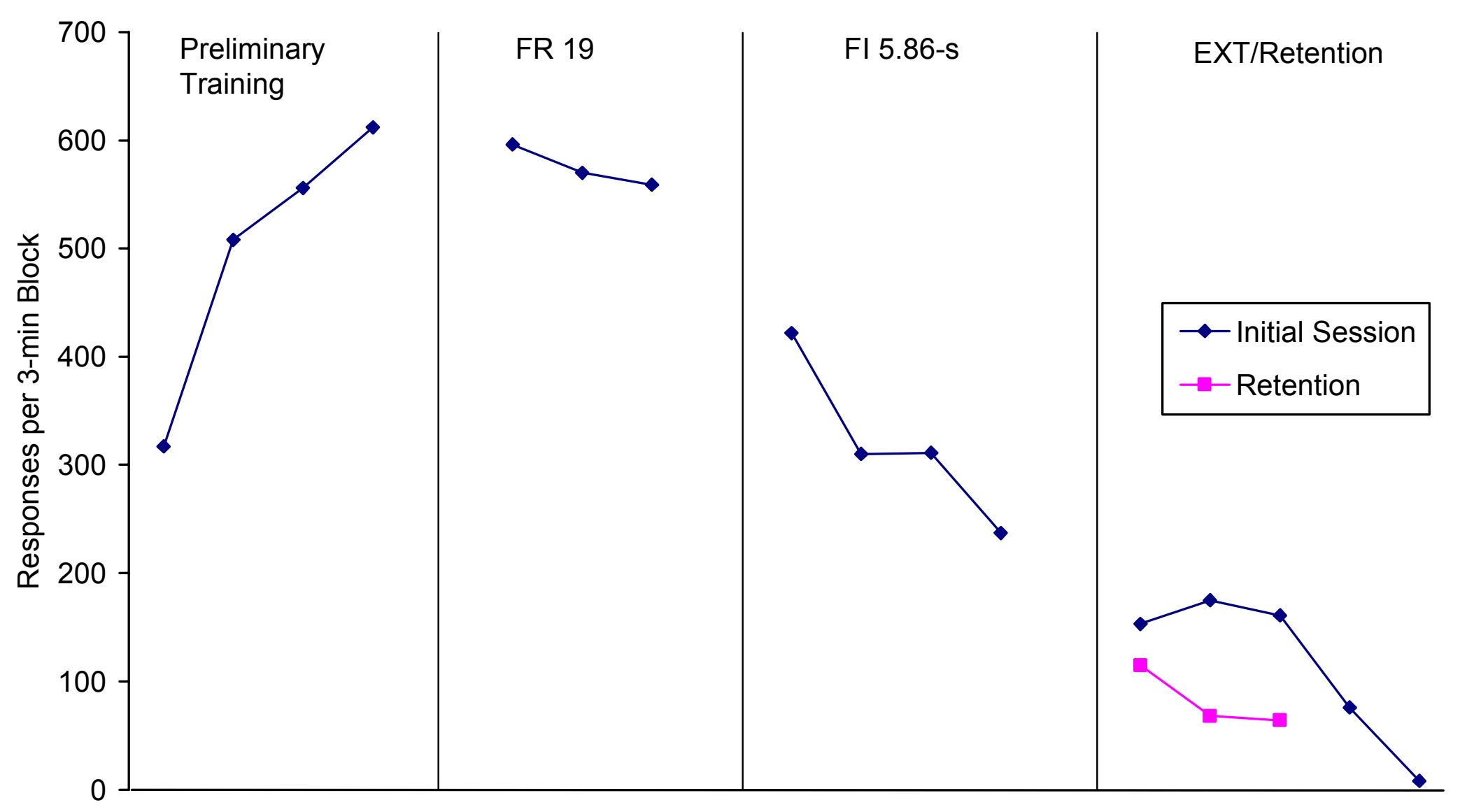


Figure 4. Response rates of participant Y17 during initial and retention sessions.

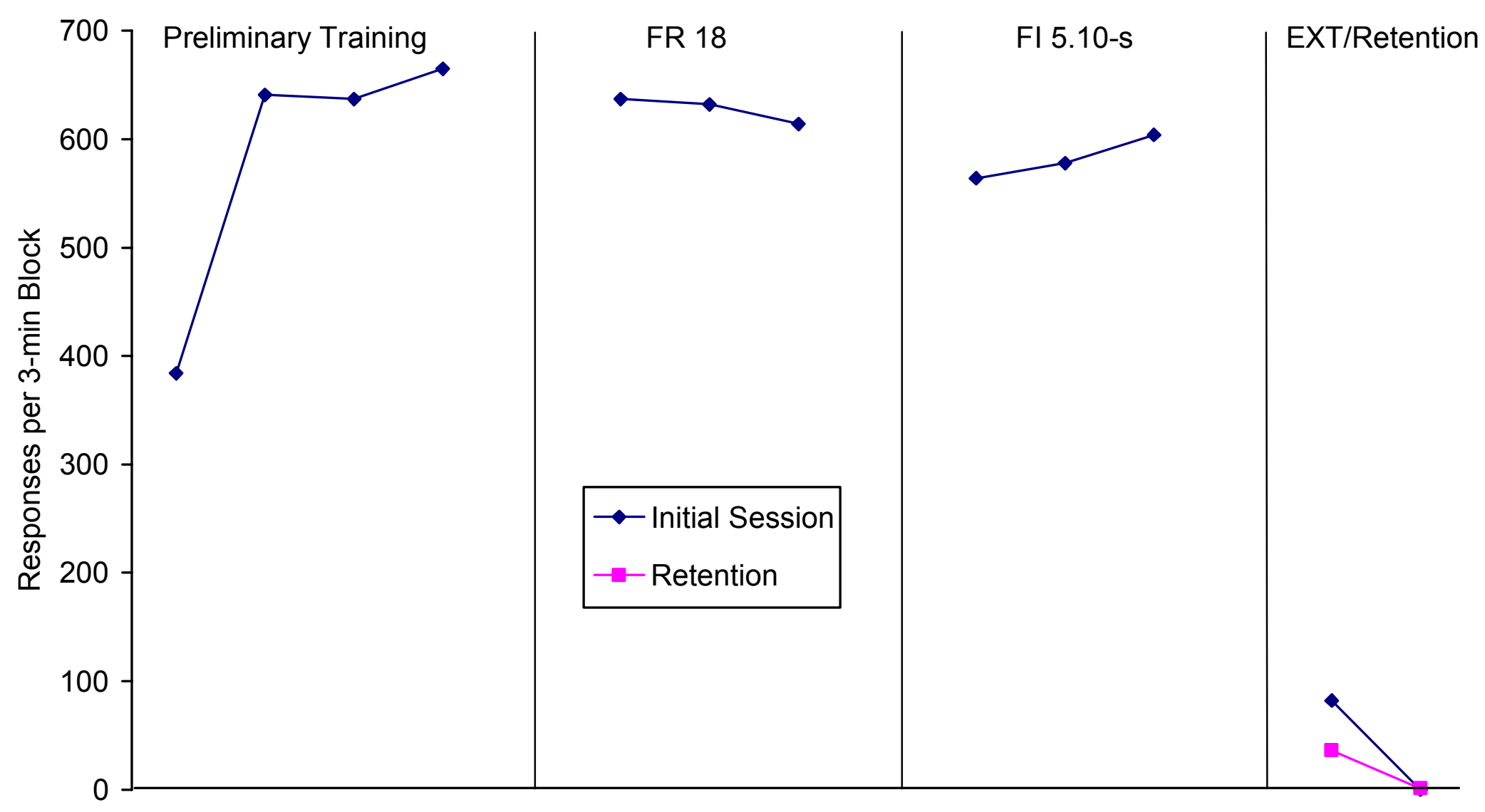


Figure 5. Response rates of participant Y18 during initial and retention sessions.

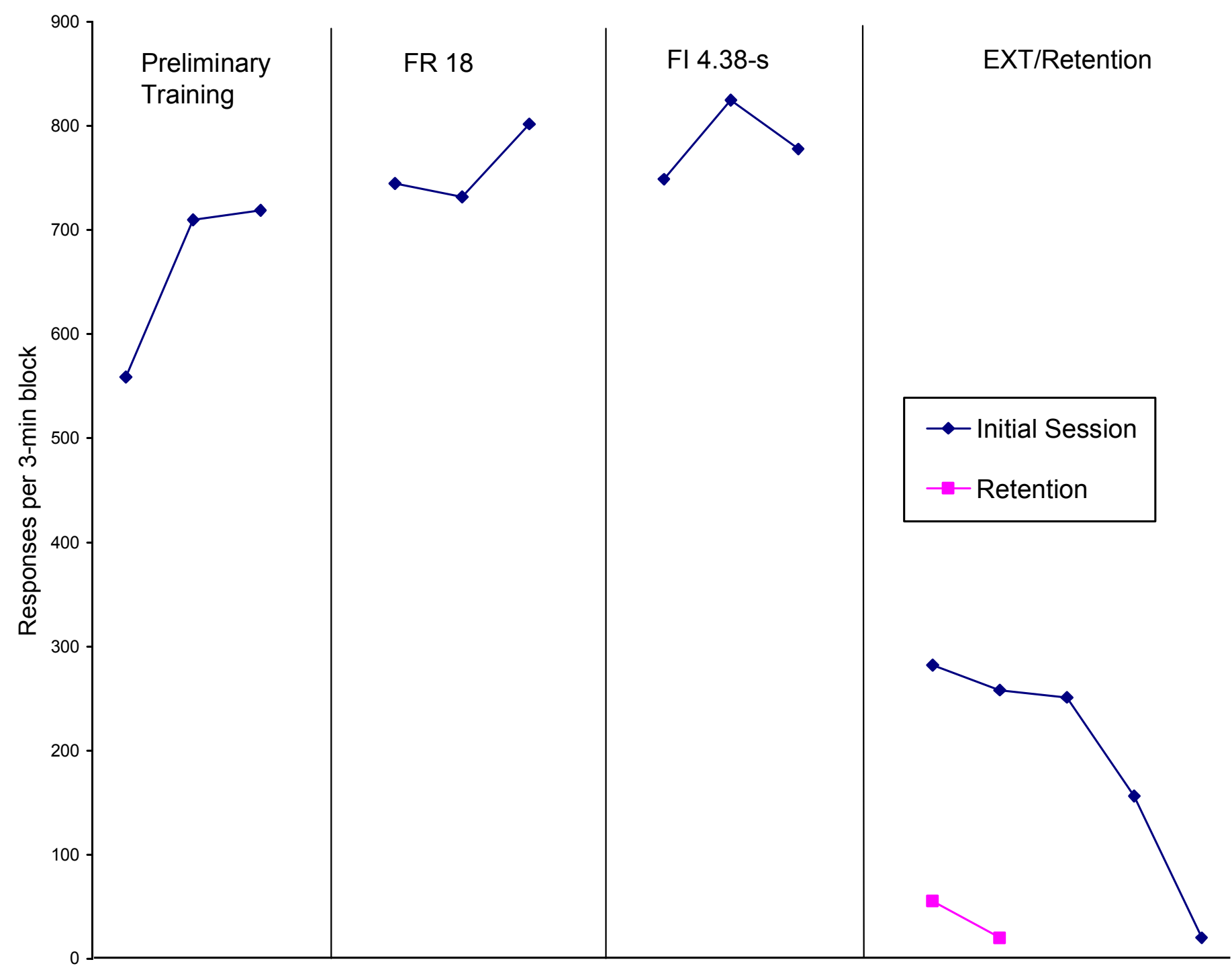


Figure 6. Response rates of participant OA27 during initial and retention sessions.

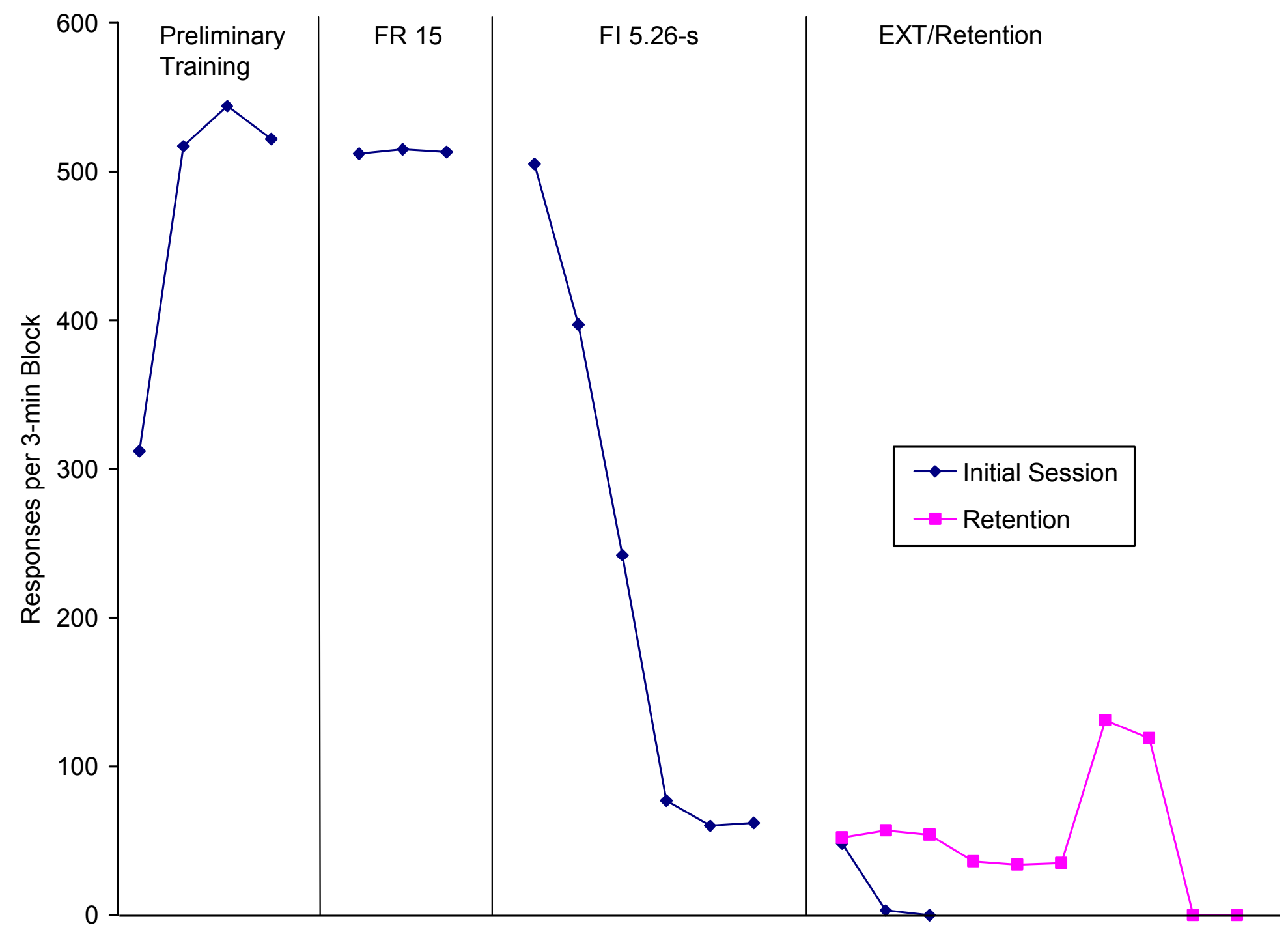


Figure 7. Response rates of participant OA13 during initial and retention sessions.

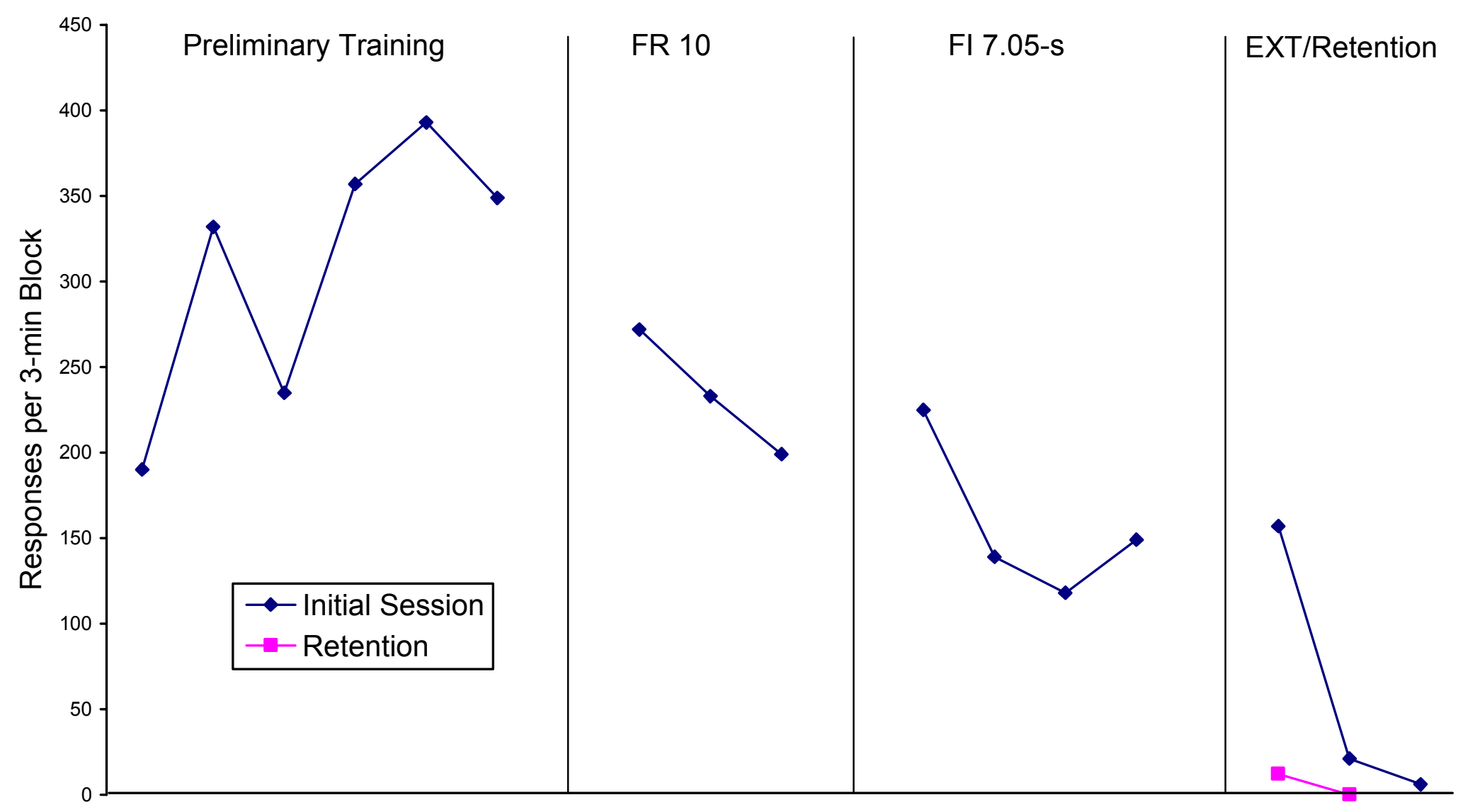


Figure 8. Response rates of participant OA20 during initial and retention sessions.

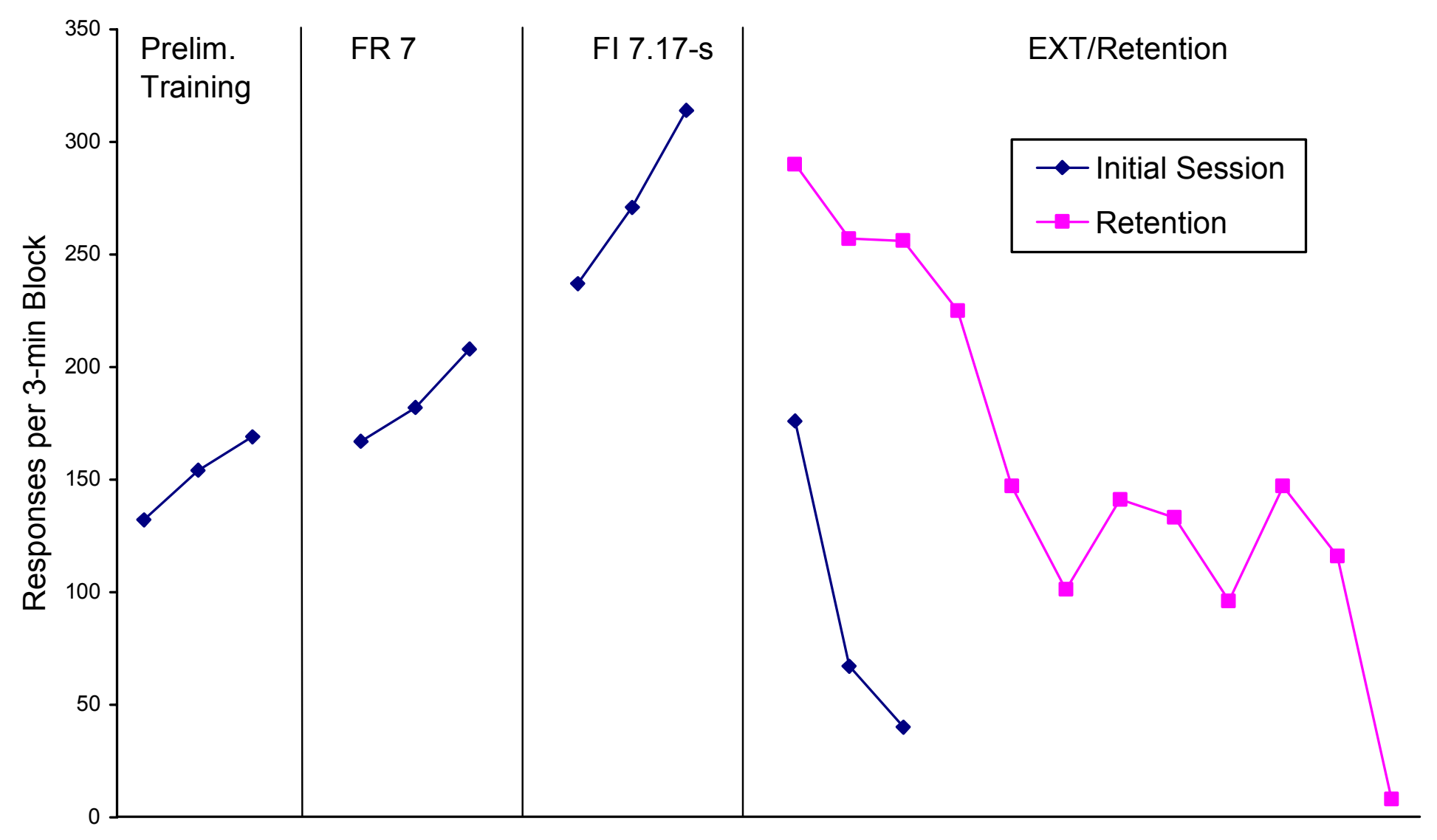


Figure 9. Response rates of participant OA26 during initial and retention sessions.

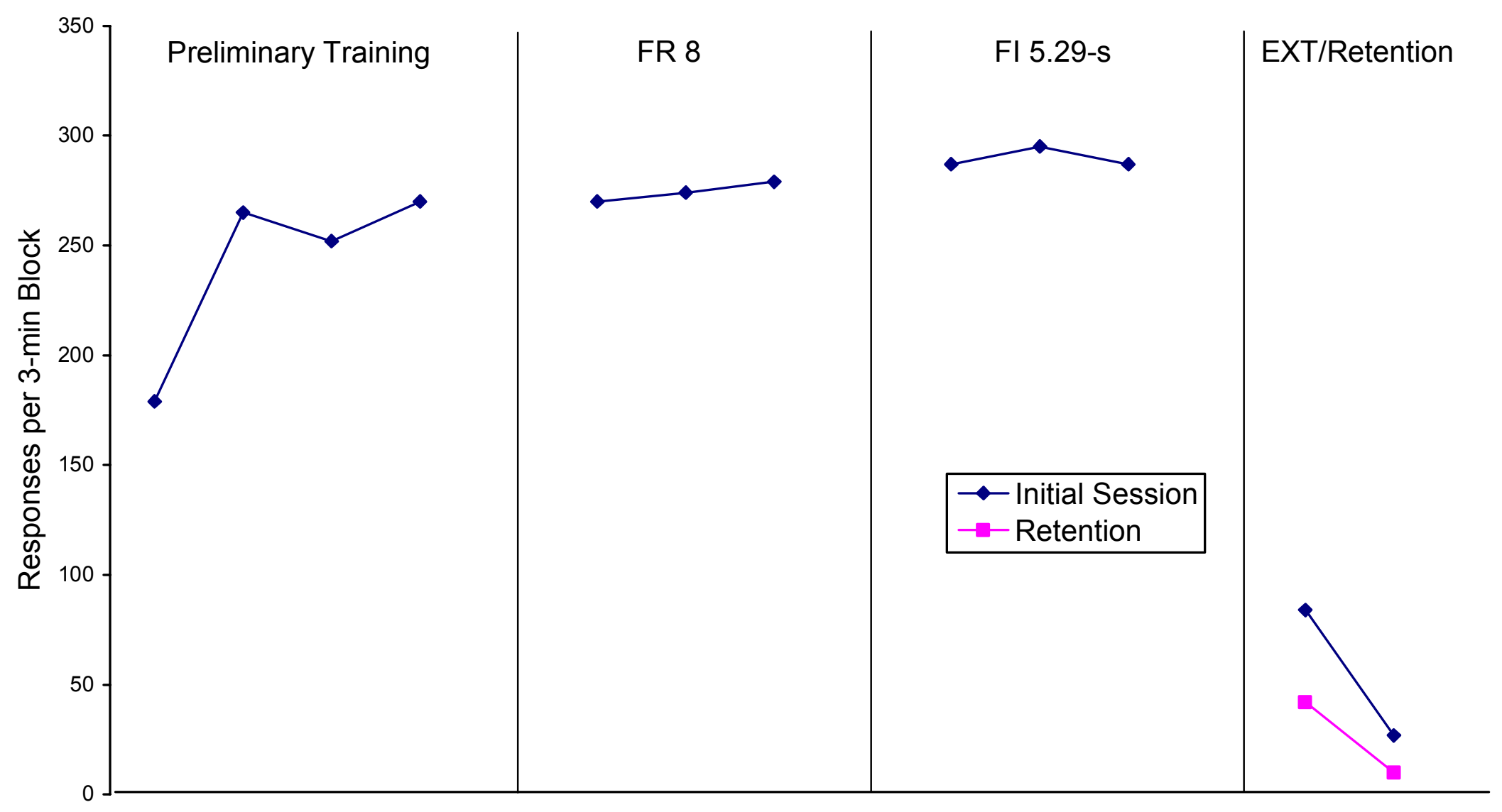


Figure 10. Response rates of participant AD23 during initial and retention sessions.

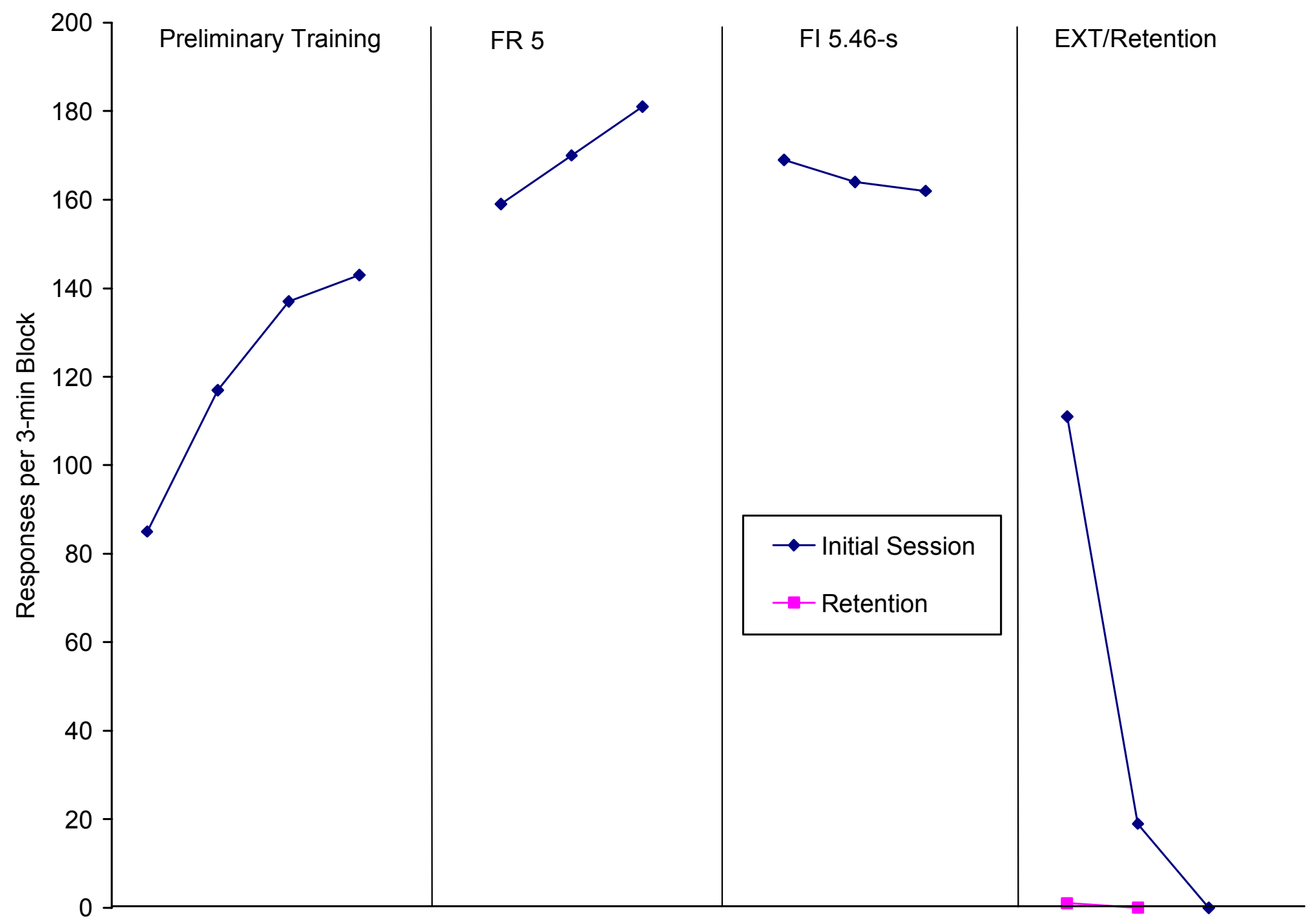


Figure 11. Response rates of participant AD30 during initial and retention sessions.

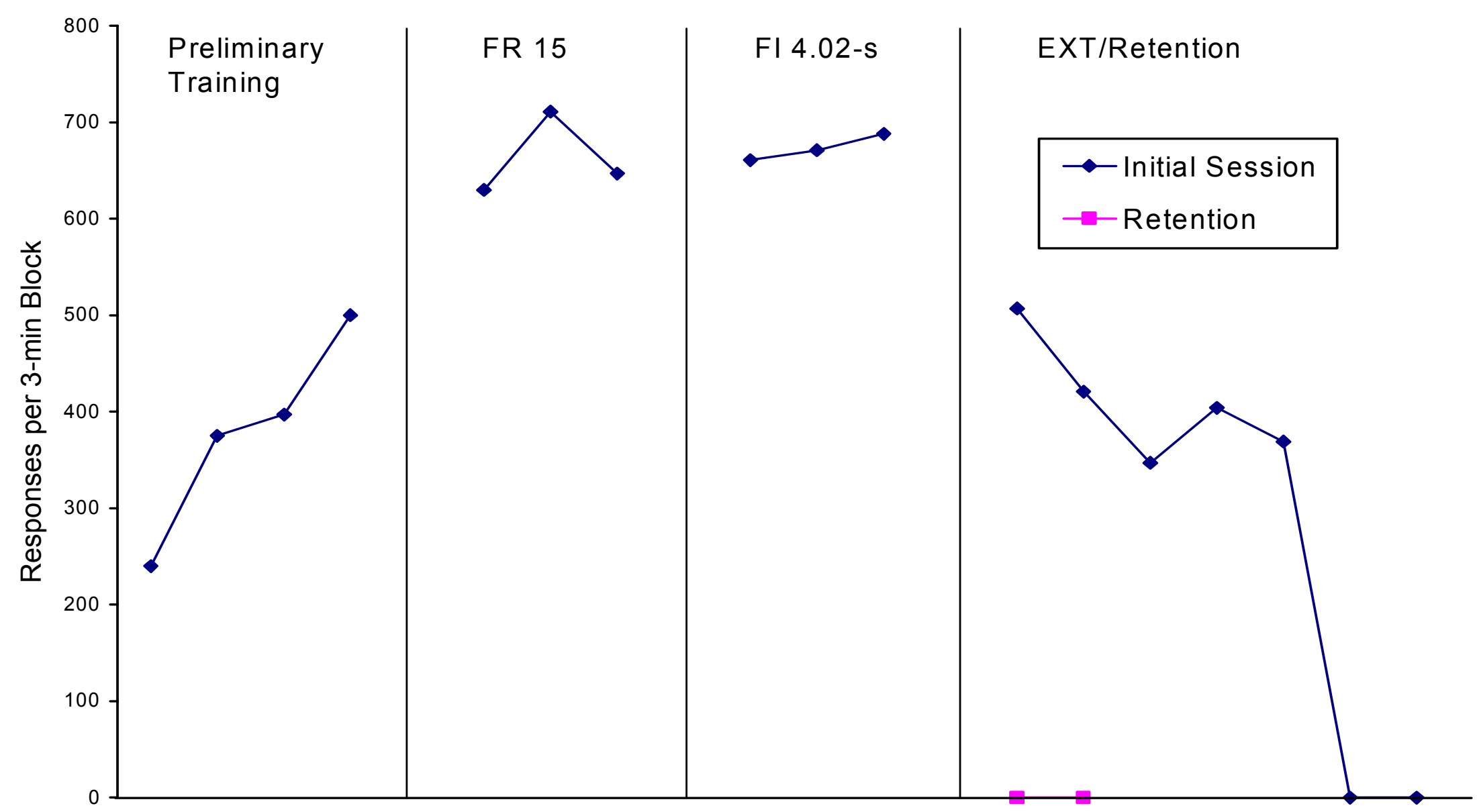


Figure 12. Response rates of participant AD31 during initial and retention sessions.

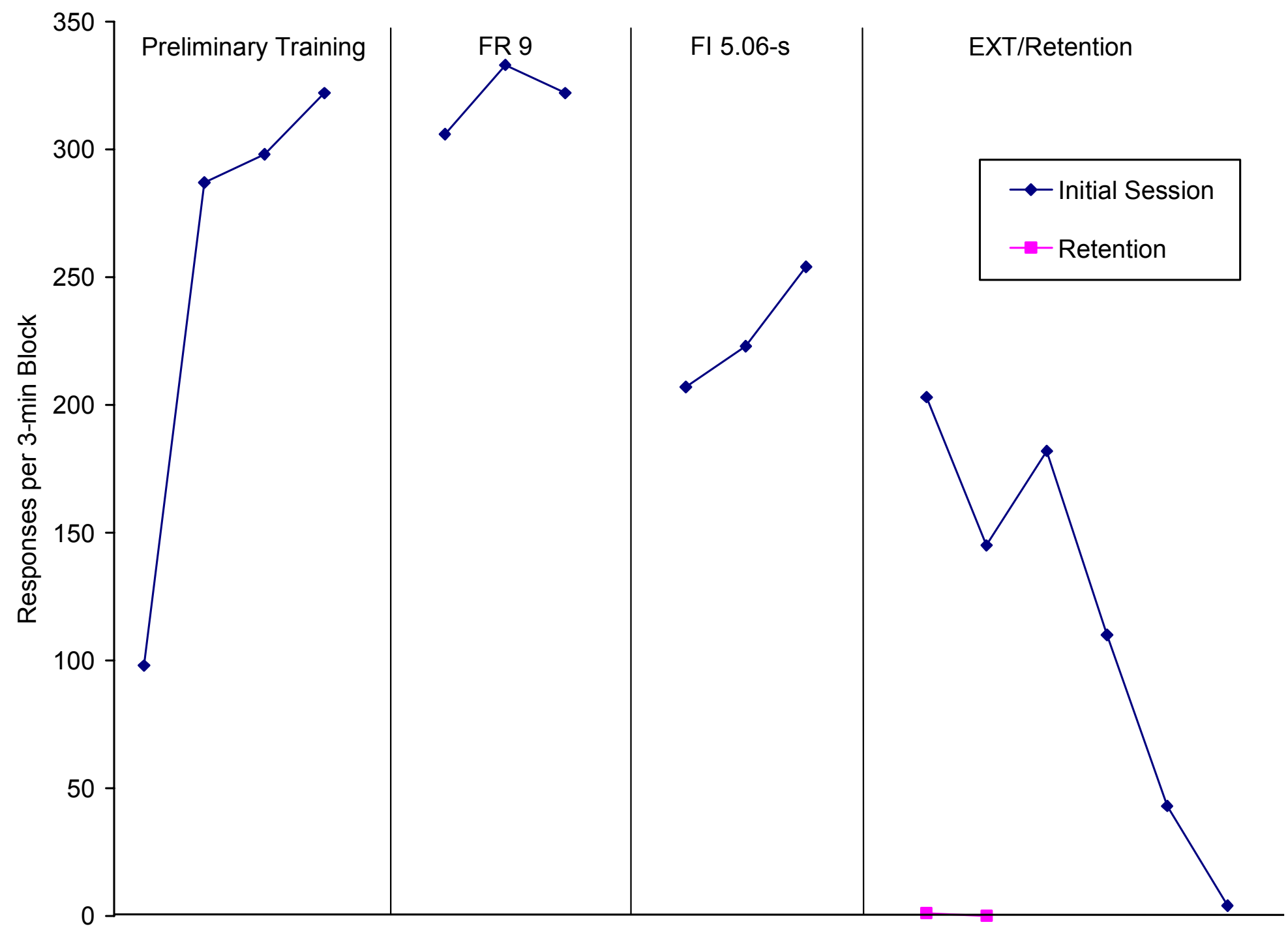




\section{Appendix A \\ NINCDS-ADRDA Diagnostic Criteria for Probable Alzheimer's Disease}

(McKhann et al., 1984, p. 940)

"I. Criteria for the clinical diagnosis of PROBABLE Alzheimer's disease include:

Dementia established by clinical examination and documented by the Mini-Mental Test; Blessed Dementia Scale, or some similar examination, and confirmed by neuropsychological tests; deficits in two or more areas of cognition; progressive worsening of memory and other cognitive functions; no disturbance of consciousness; onset between ages 40 and 90, most often after age 65; and absence of systemic disorders or other brain diseases that in and of themselves could account for the progressive deficits in memory and cognition.

II. The diagnosis of PROBABLE Alzheimer`s disease is supported by:

Progressive deterioration of specific cognitive functions such as language (aphasia), motor skills (apraxia), and perception (agnosia); impaired activities of daily living and altered patterns of behavior; family history of similar disorders, particularly if confirmed neuropathologically; and laboratory results of: normal lumbar puncture as evaluated by standard techniques, normal pattern or non-specific changes in EEG, such as increased slowwave activity, and evidence of cerebral atrophy on CT with progression documented by serial observation.

III. Other clinical features consistent with the diagnosis of PROBABLE Alzheimer`s disease, after exclusion of causes of dementia other than Alzheimer's disease, include:

Plateaus in the course of progression of the illness; associated symptoms of depression, insomnia, incontinence, delusions, illusions, hallucinations, catastrophic verbal, emotional, or physical outbursts, sexual disorders, and weight loss; other neurologic abnormalities in some patients, especially with more advanced disease and including motor signs such as increased muscle tone, myoclonus, or gait disorder; seizures in advanced disease; and CT normal for age.

IV. Features that make the diagnosis of PROBABLE Alzheimer`s disease uncertain or unlikely include:

Sudden, apoplectic onset; focal neurologic findings such as hemiparesis, sensory loss, visual field deficits, and incoordination early in the course of the illness; and seizures or gait disturbances at the onset or very early in the course of the illness." 
Appendix B

Clinical Interview

\section{Psychotic Disorders}

Do you ever see things or hear things that other people can't hear or see?

Do believe that other people are plotting against you?

Do you ever think that someone on the TV or radio is speaking especially for or about you?

Can other people steal your thoughts or plant thoughts inside your mind?

Do you have any beliefs that others might consider unusual or strange?

\section{Mood Disorders}

Do you ever feel very sad for days on end?

Do you find yourself thinking that you would be better off dead?

Do you ever feel very happy, excited, irritable, or restless for an extended period of time?

During these times, do you spend too much money, start big projects, or have sexual contact with a lot of people?

\section{Anxiety Disorders}

Do you worry too much?

Do you ever have sudden, intense attacks of fear that come 'out of the blue?'

Are you afraid of making a fool of yourself around other people?

Do you have an intense fear of snakes, heights, dogs, or any other objects or situations?

Do you find yourself reliving any terrible events (e.g., accidents, combat, other violence) that you might have witnessed?

Do you ever find yourself doing things over and over again (e.g., washing hands, checking stove), even though you don't really want to?

Do you have disturbing thoughts?

Do you do anything to try to make those thoughts go away?

\section{Substance Abuse}

Has your consumption of alcohol or drugs ever caused problems for you?

\section{Eating Disorders}

Do you worry a lot about your weight?

Do you ever eat a very large amount of food in a short period of time?

During those times, do you ever force yourself to vomit, or abuse laxatives to prevent yourself from gaining weight?

Do you ever severely limit what you will eat because you're afraid of gaining too much weight? 
80

Appendix C: Cumulative record of participant Y10's responding during initial session.

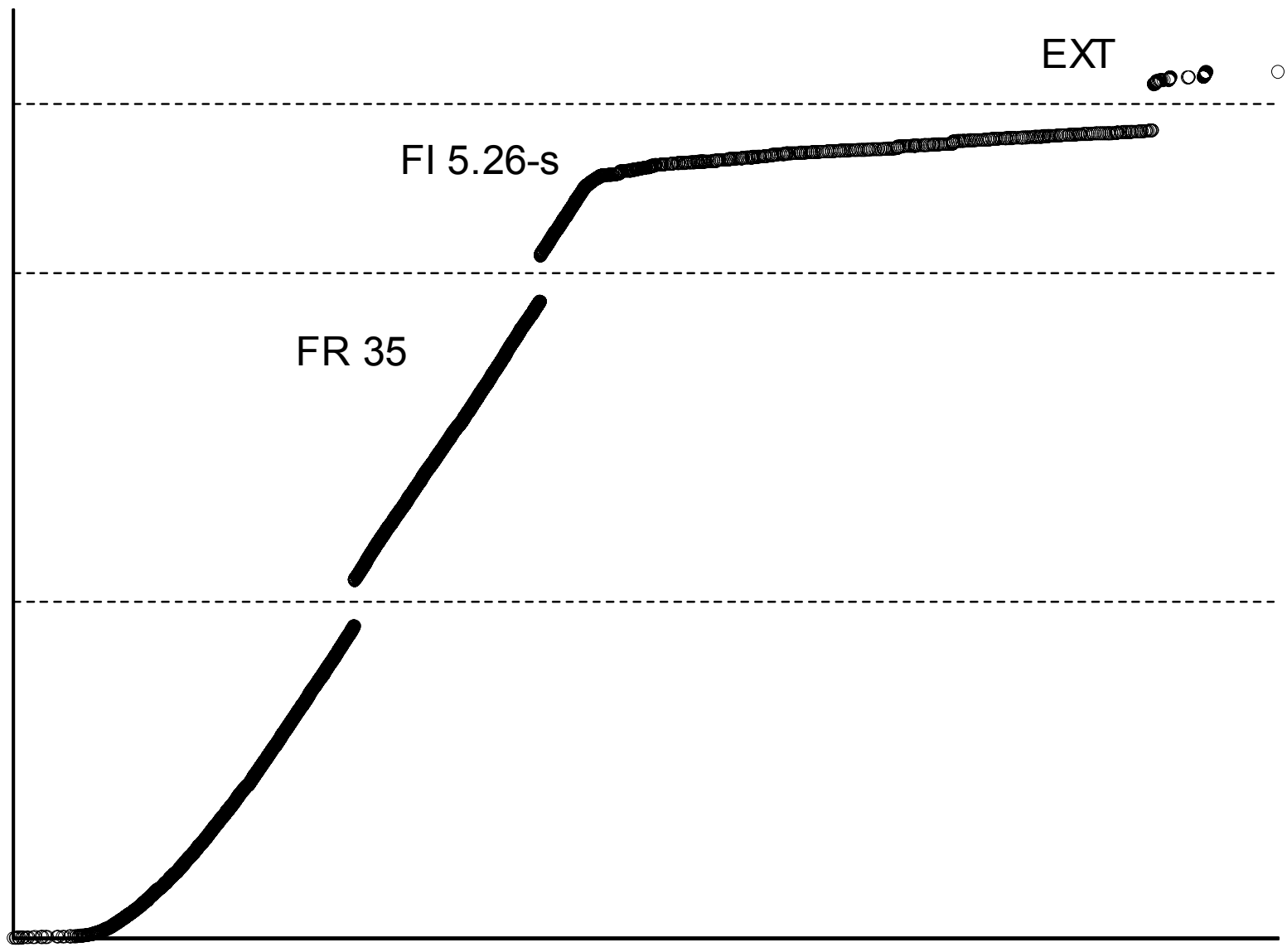

Time $\rightarrow$ 
Appendix D: Cumulative record of participant Y22's responding during initial session.

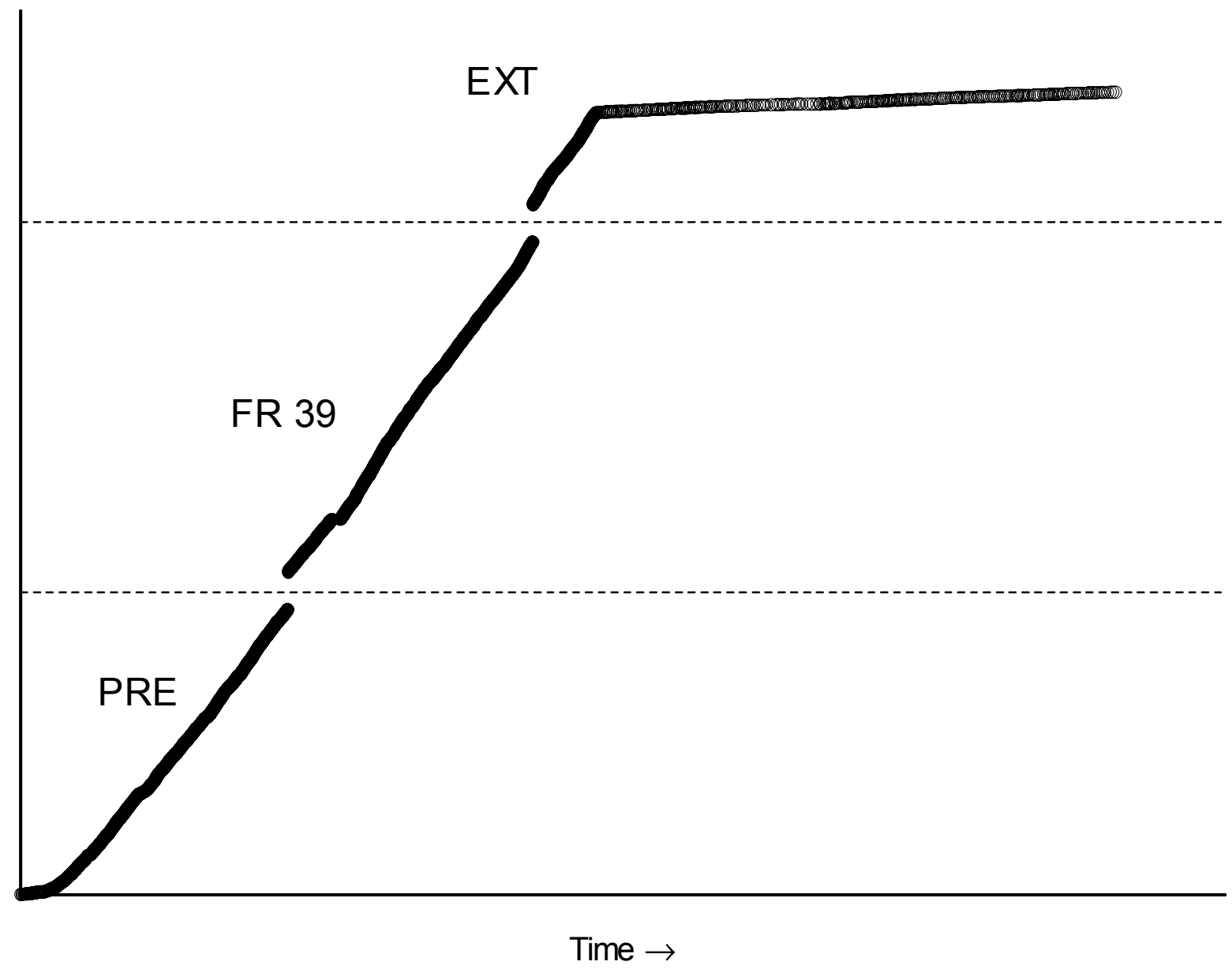


Appendix E: Cumulative record of participant Y21's responding during initial session.

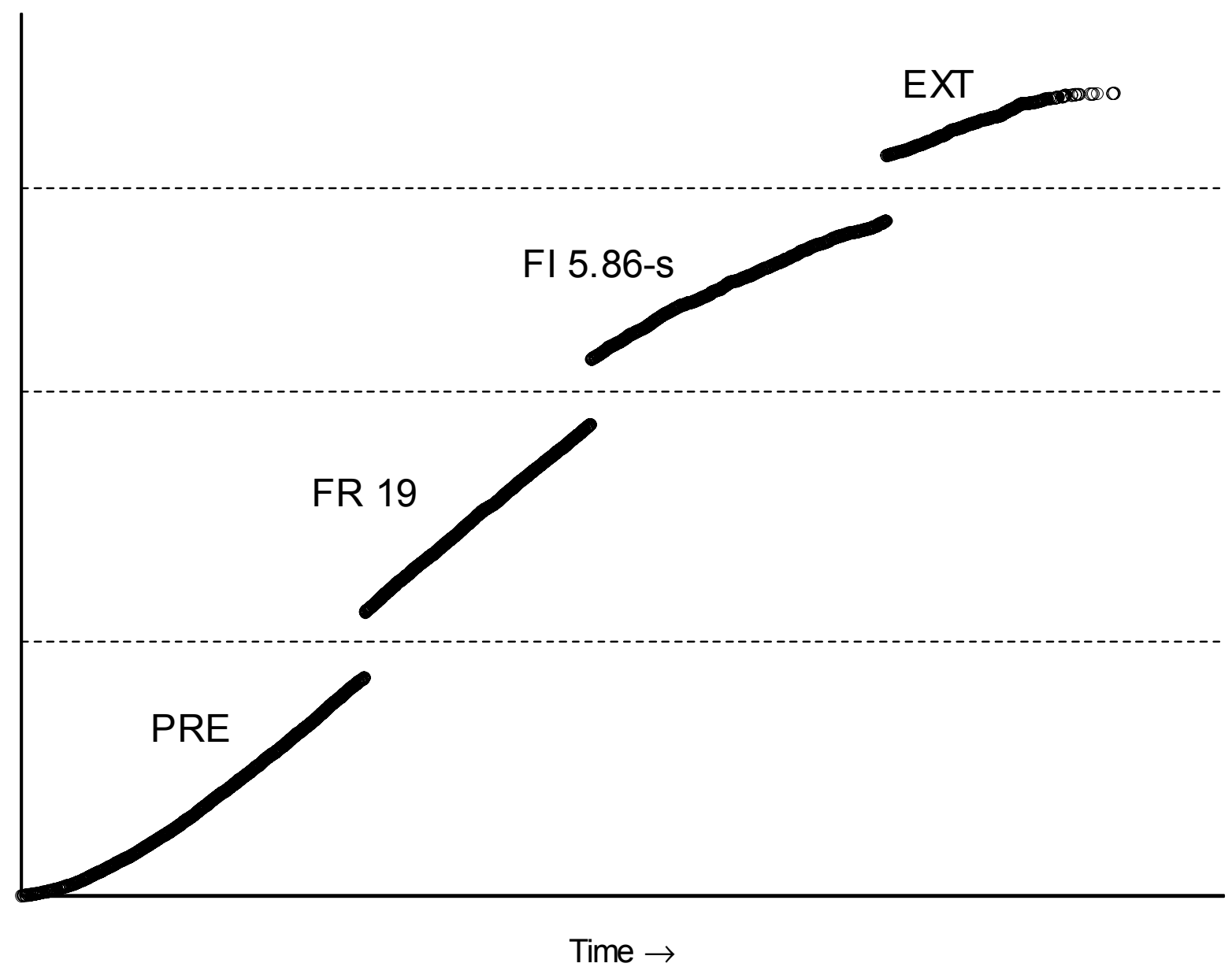


83

Appendix F: Cumulative record of participant Y17's responding during initial session.

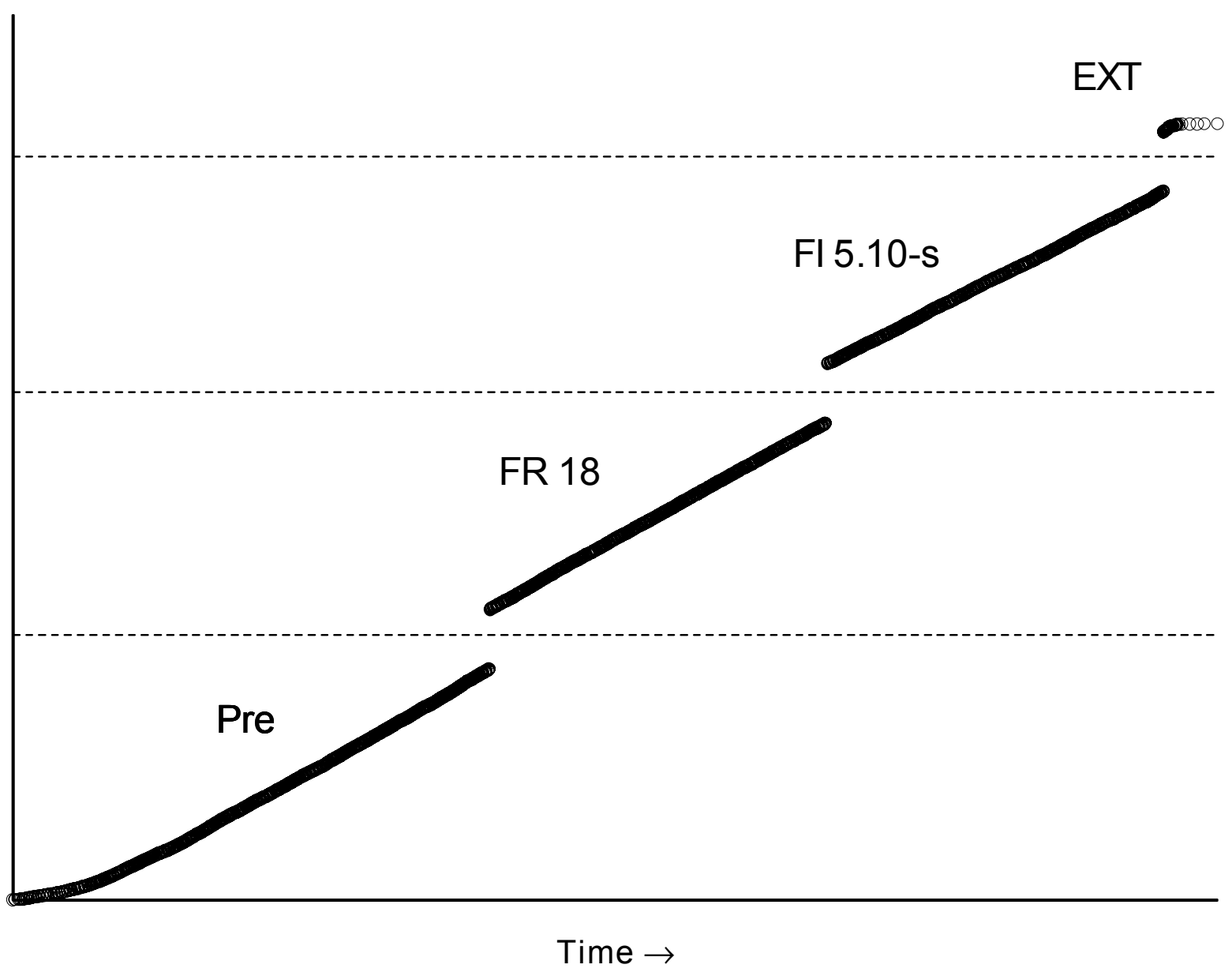


84

Appendix G: Cumulative record of participant Y18's responding during initial session.

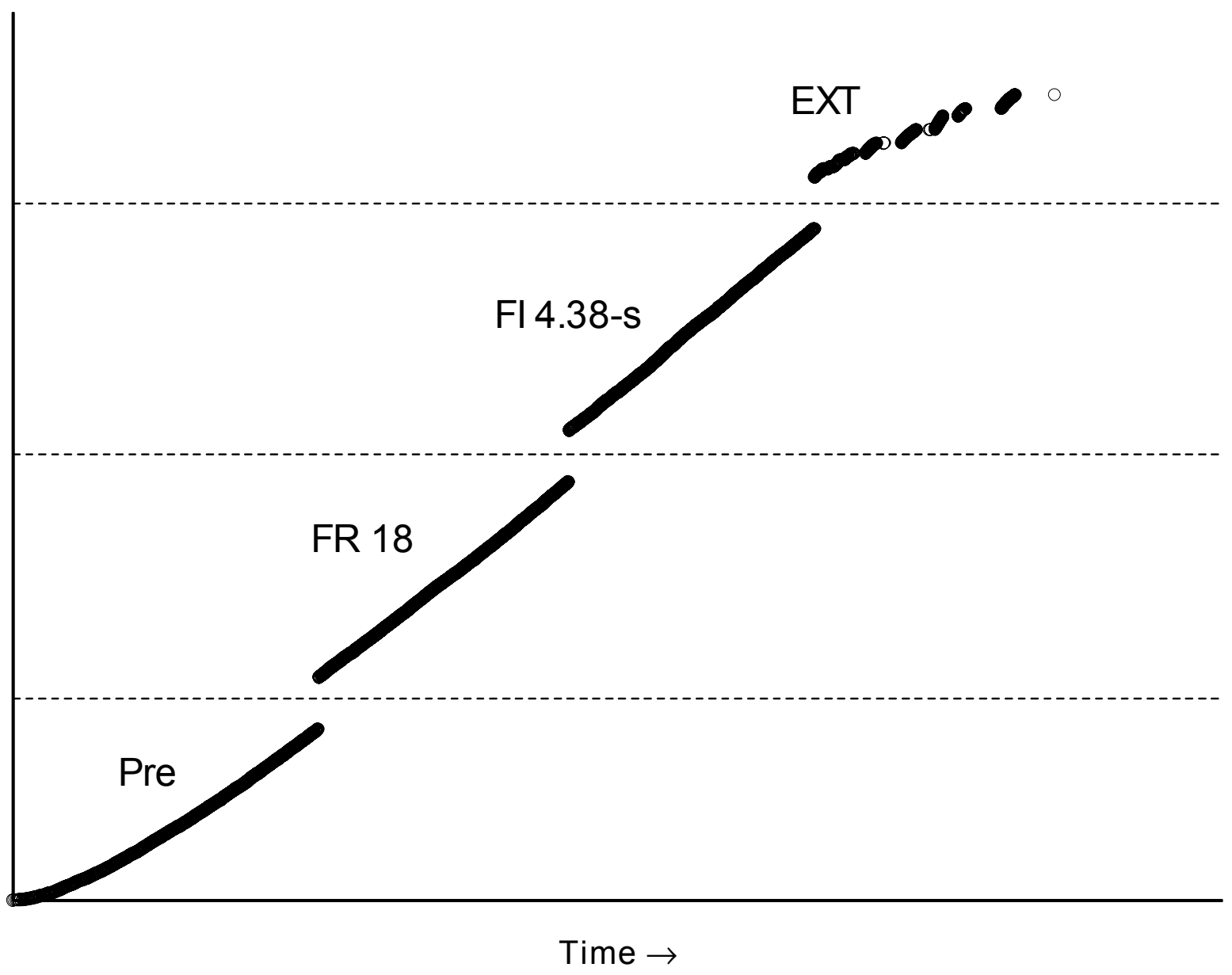


85

Appendix H: Cumulative record of participant OA27's responding during initial session.

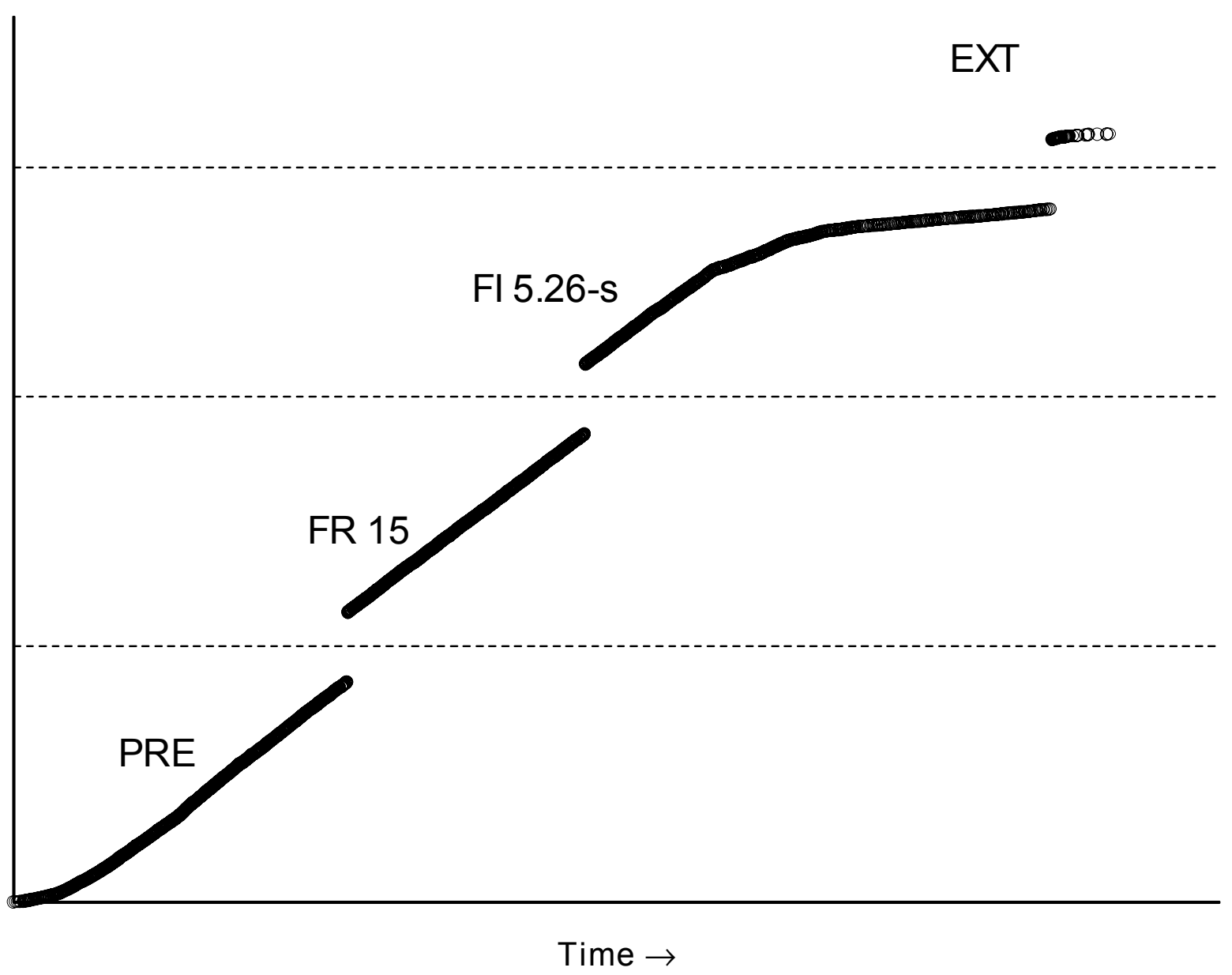


86

Appendix I: Cumulative record of participant OA13's responding during initial session.

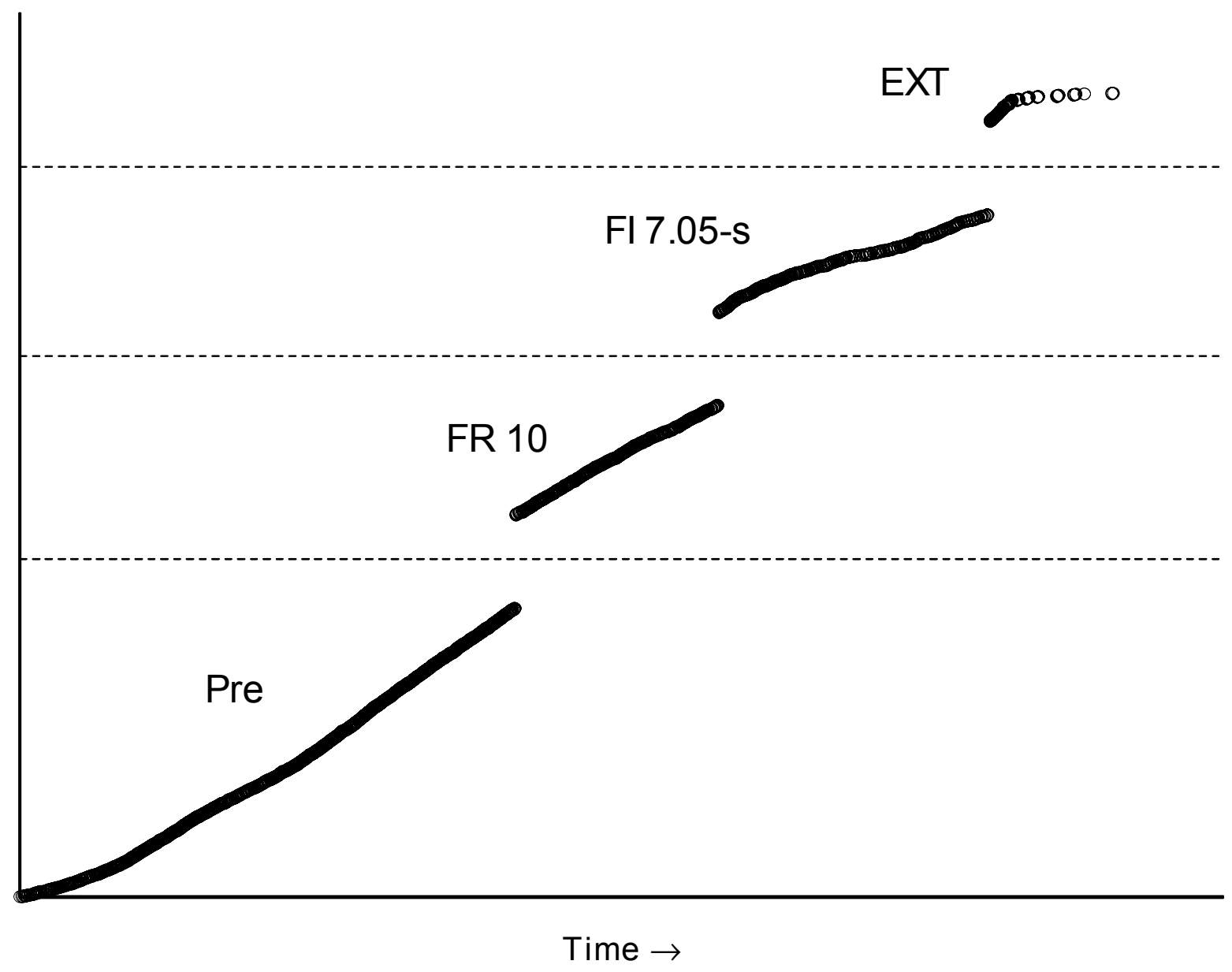


87

Appendix J: Cumulative record of participant OA20's responding during initial session.

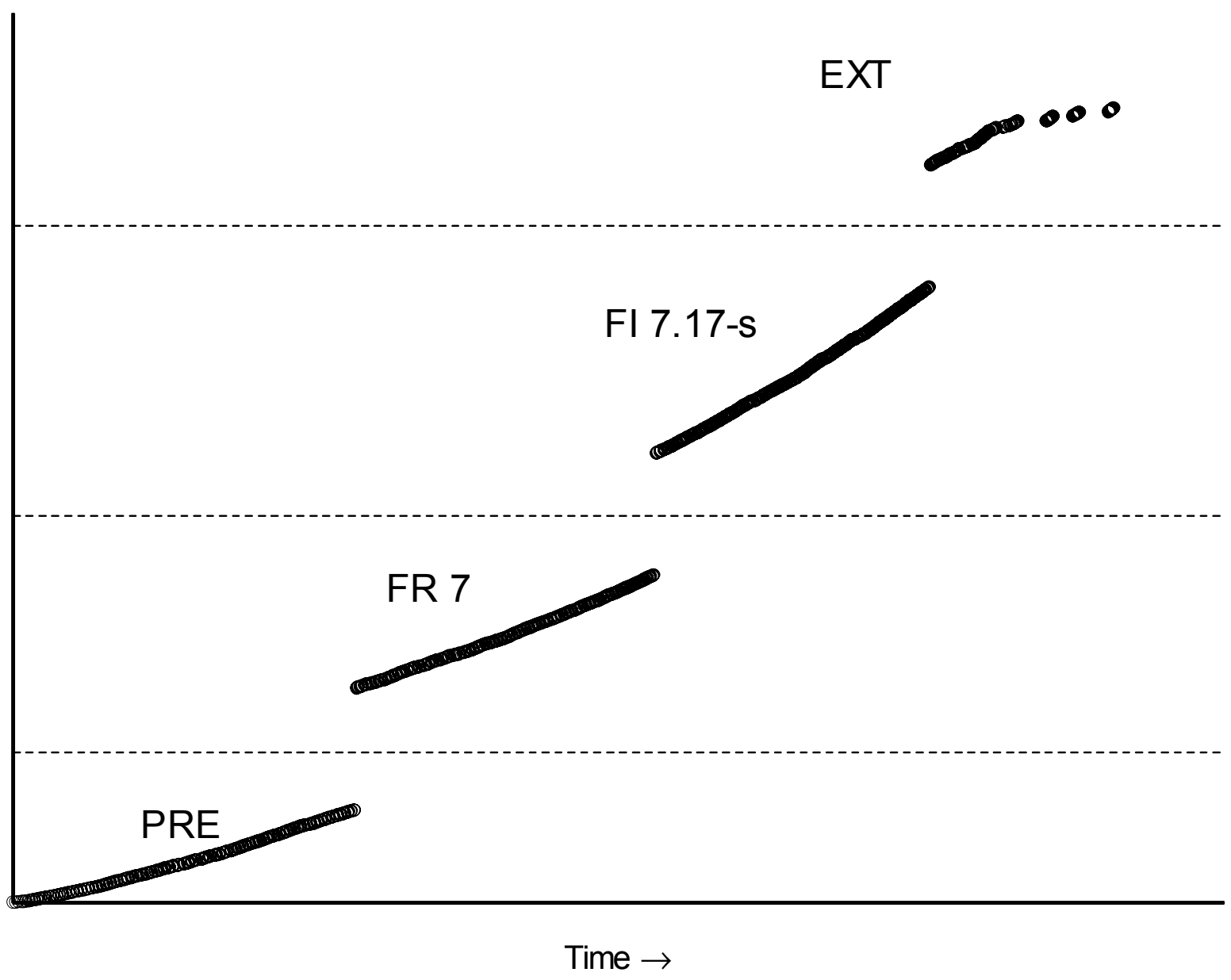


88

Appendix K: Cumulative record of participant OA26's responding during initial session.

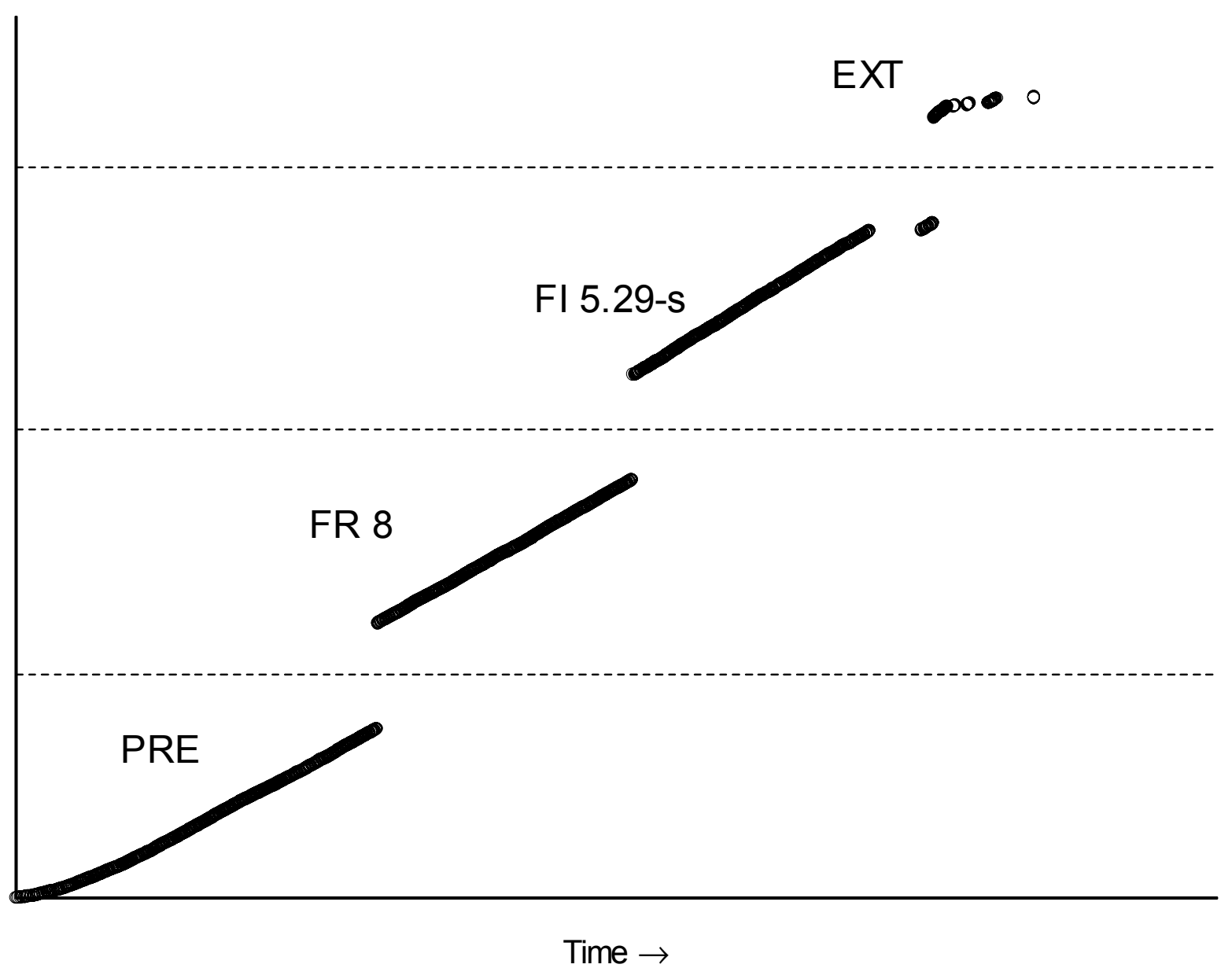


89

Appendix L: Cumulative record of participant AD23's responding during initial session.

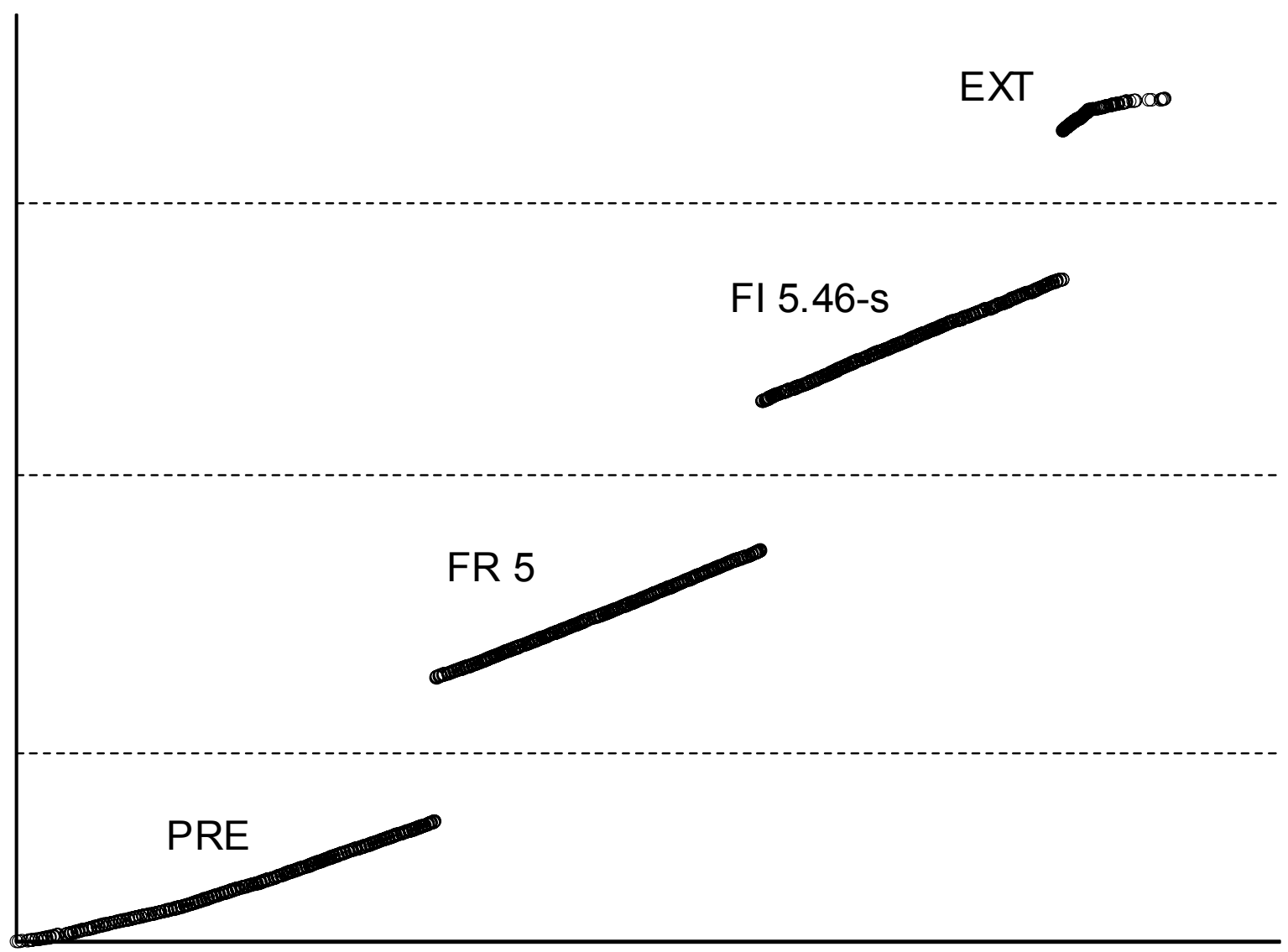

Time $\rightarrow$ 
Appendix M: Cumulative record of participant AD30's responding during initial session.

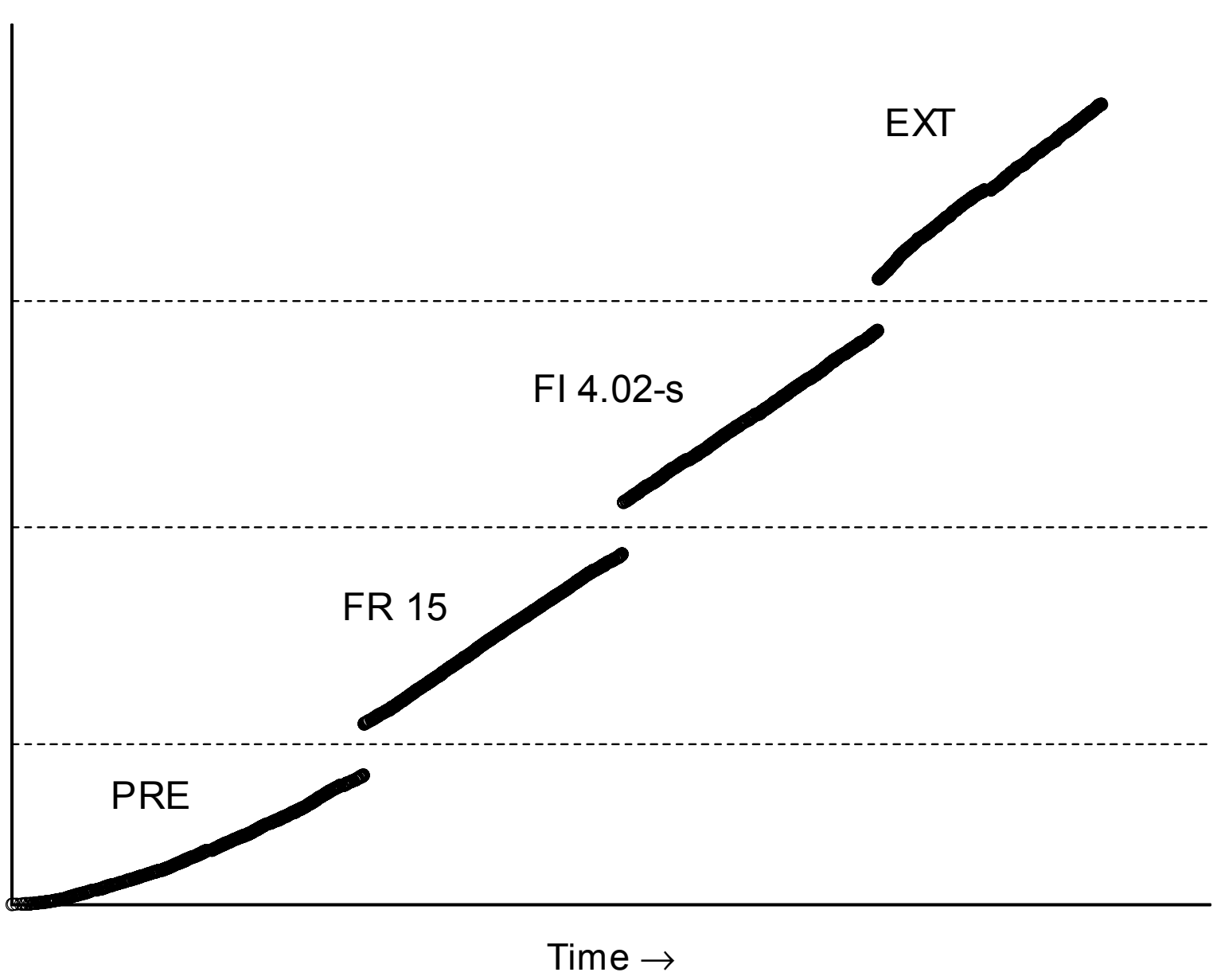


91

Appendix N: Cumulative record of participant AD 31's responding during initial session.

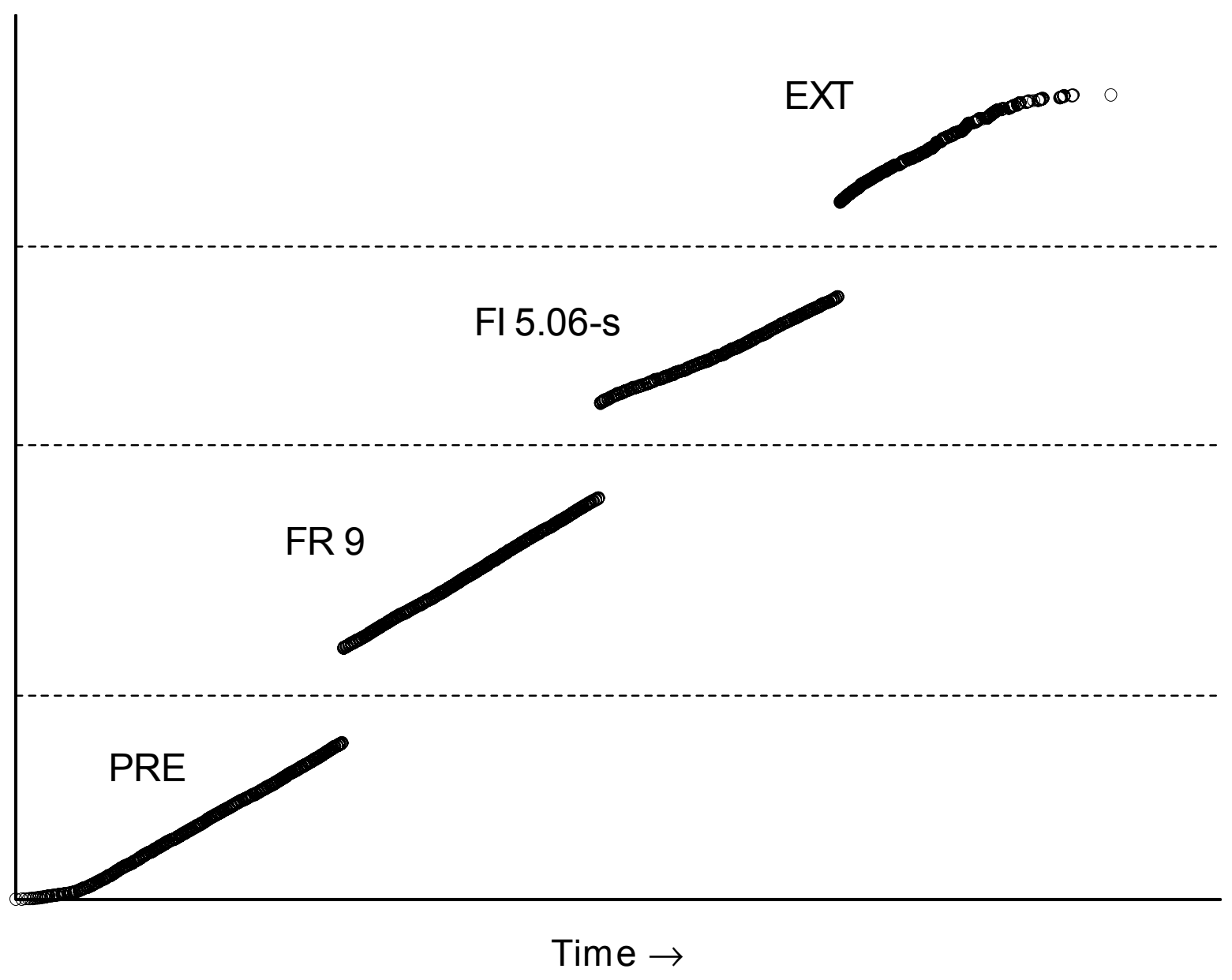


92

Appendix O: Cumulative record of participant AD28 during preliminary training and FR conditions.

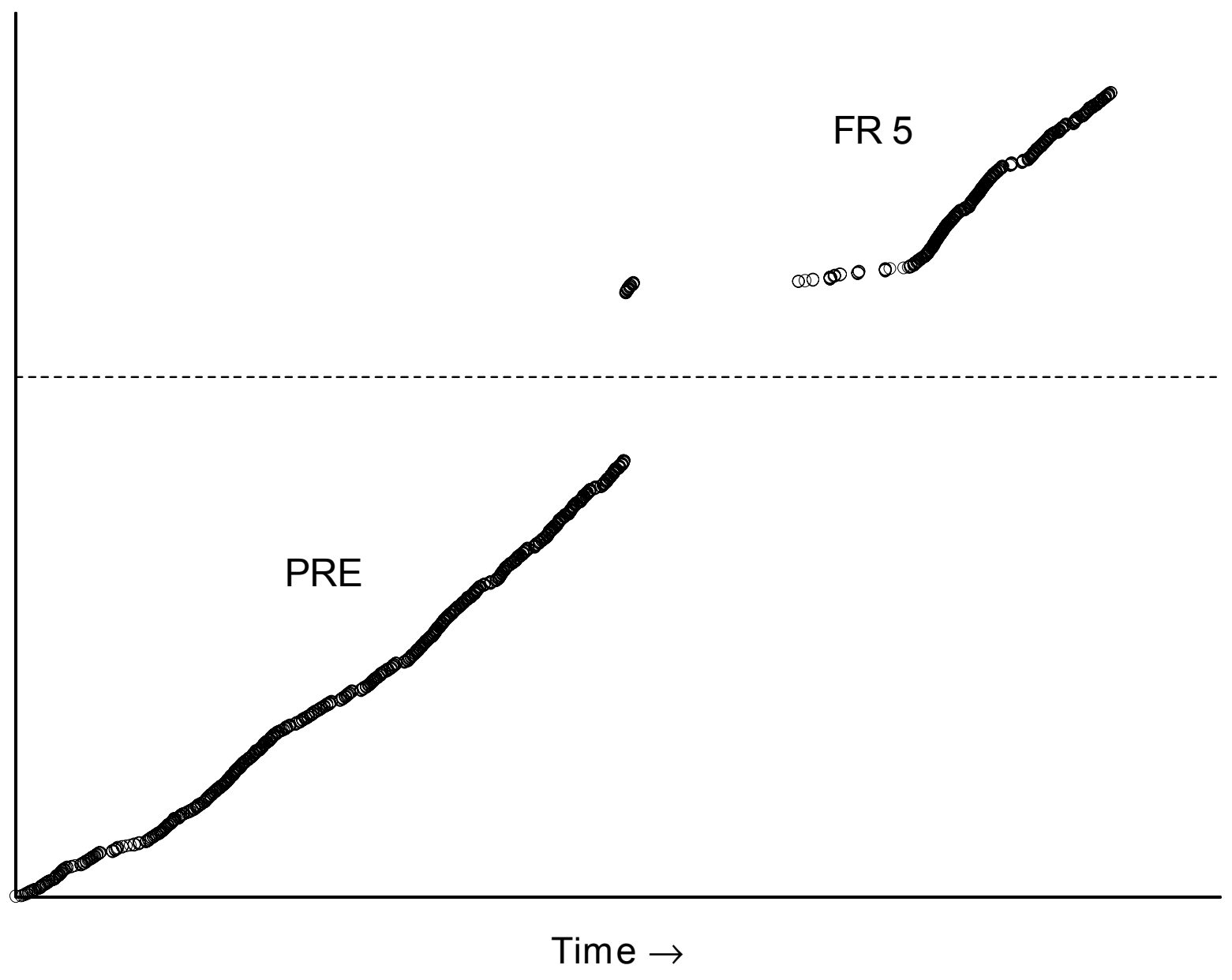

